\title{
The what and when in brain stimulation : studying language production and deception with optimised neuronavigated transcranial magnetic stimulation
}

Citation for published version (APA):

Schuhmann, T. (2010). The what and when in brain stimulation : studying language production and deception with optimised neuronavigated transcranial magnetic stimulation. [Doctoral Thesis, Maastricht University]. Universitaire Pers Maastricht. https://doi.org/10.26481/dis.20100603ts

Document status and date:

Published: 01/01/2010

DOI:

$10.26481 /$ dis.20100603ts

Document Version:

Publisher's PDF, also known as Version of record

Please check the document version of this publication:

- A submitted manuscript is the version of the article upon submission and before peer-review. There can be important differences between the submitted version and the official published version of record.

People interested in the research are advised to contact the author for the final version of the publication, or visit the DOI to the publisher's website.

- The final author version and the galley proof are versions of the publication after peer review.

- The final published version features the final layout of the paper including the volume, issue and page numbers.

Link to publication

\footnotetext{
General rights rights.

- You may freely distribute the URL identifying the publication in the public portal. please follow below link for the End User Agreement:

www.umlib.nl/taverne-license

Take down policy

If you believe that this document breaches copyright please contact us at:

repository@maastrichtuniversity.nl

providing details and we will investigate your claim.
}

Copyright and moral rights for the publications made accessible in the public portal are retained by the authors and/or other copyright owners and it is a condition of accessing publications that users recognise and abide by the legal requirements associated with these

- Users may download and print one copy of any publication from the public portal for the purpose of private study or research.

- You may not further distribute the material or use it for any profit-making activity or commercial gain

If the publication is distributed under the terms of Article $25 \mathrm{fa}$ of the Dutch Copyright Act, indicated by the "Taverne" license above, 


\section{THE WHAT AND WHEN IN BRAIN STIMULATION}

STUDYING LANGUAGE PRODUCTION AND DECEPTION WITH OPTIMISED NEURONAVIGATED TRANSCRANIAL MAGNETIC STIMULATION 
Production: Datawyse / Universitaire Pers Maastricht Copyright (C) Teresa Schuhmann, Maastricht, 2010

ISBN 9789052789576

The work presented in this thesis was funded by the Netherlands Organization for Scientific Research (NWO) and was conducted at Maastricht University. 


\title{
THE WHAT AND WHEN IN BRAIN STIMULATION
}

\author{
STUDYING LANGUAGE PRODUCTION AND DECEPTION \\ WITH OPTIMISED NEURONAVIGATED \\ TRANSCRANIAL MAGNETIC STIMULATION
}

PROEFSCHRIFT

Ter verkrijging van de graad van doctor aan de Universiteit Maastricht, op gezag van de Rector Magnificus, prof. mr. G.P.M.F. Mols volgens het besluit van het College van Decanen,

in het openbaar te verdedigen op donderdag 3 juni 2010 om 12:00 uur

door

\section{Teresa Schuhmann}

Geboren op 15 september 1979 te Scherzingen, Zwitserland

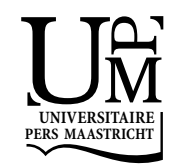




\section{Promotor}

Prof. dr. Niels O. Schiller, Universiteit Leiden

\section{Copromotor}

Dr. Alexander T. Sack

\section{Beoordelingscommissie}

Prof. dr. Bernadette Jansma (voorzitter)

Dr. Joan A. Camprodon (Harvard University, USA)

Prof. dr. Felix Mottaghy (RWTH Aachen University, Germany)

Dr. Vincent van den Ven 
Meinen Eltern 

Chapter 2 Optimising functional accuracy of TMS in cognitive studies:

A comparison of methods

Chapter 3 The temporal characteristics of functional activation in Broca's area during overt picture naming

Chapter 4 Speaking of which: Dissecting the neurocognitive network of language production

Chapter 5 The functional role of inferior frontal sulcus in deception:

A neuronavigated theta-burst transcranial magnetic stimulation study

Summary and Conclusion

Samenvatting en Conclusie

Dankwoord

Curriculum Vitae

Publications 



\section{CHAPTER 1}

\section{GENERAL INTRODUCTION}





\section{COGNITIVE NEUROSCIENCE}

Cognitive neuroscience is based on the principle that every aspect of behaviour, perception, and cognition reflects an organised neuronal activation in the brain. Today, in a multi- and interdisciplinary endeavour, the conceptual models of cognition and information processing provided by cognitive psychology are combined with the new technical developments in functional neuroimaging in order to explore the organisation and functional structure of cognitive functions in the human brain. Researchers representing different scientific disciplines such as psychology, engineering, medicine, physics, biology, mathematics or computer science are now working together in the new emerging field of cognitive neuroscience, in which each of these scientific disciplines contribute their respective genuine theories, models, techniques and tools for the mutual investigation of the neuronal principles of behaviour, perception and cognition.

Among the list of main objectives in cognitive neuroscience are questions concerning the location, function and temporal sequence of cortical activities in relation to behaviour and cognition. A large number of studies in empirical brain research, and especially those using techniques of functional imaging, have provided information on the exact spatial layout of different perceptual and cognitive functions in the healthy human brain. A key objective of cognitive neuroscience is to elucidate causal relationships between brain activations and perception, behaviour or cognition. Cognitive neuroscientists recognise that, besides the mere localisation of brain functions, an understanding of the performance of even the simplest task requires the analysis of the different components of its execution, its functional connectivity and interactions in different regions of the brain.

Several neuroimaging studies have been conducted to investigate these functional neuronal correlates of human elementary perceptual processing, motor control, emotions and other cognitive functions in vivo. The application of imaging techniques to the investigation of brain areas activated during ever more elaborate experimental paradigms has increased extensively in the last ten years. Since its application to cognitive science, functional imaging has opened the door to the observation of functional brain organisation.

\section{METHODS IN COGNITIVE NEUROSCIENCE}

The last decades have seen the development of various new methods within cognitive neuroscience. These methods can broadly be divided into structural and functional brain imaging techniques. Structural imaging techniques such as computed tomography (CT) or magnetic resonance imaging (MRI) are able to visualise the anatomical structures of the human brain. While CT is an advanced version of the conventional $x$-ray study allowing for the reconstruction of three-dimensional space from the compressed two-dimensional images, MRI exploits the magnetic properties of organic tissue to non-invasively obtain high resolution images of human anatomy (for details see next section). 
Functional imaging techniques, on the other hand, are capable of also detecting or measuring changes in metabolism, blood flow, regional chemical composition and absorption, that are associated with the execution of a given cognitive task. As opposed to structural imaging, functional imaging thus focuses on revealing taskcorrelated physiological activities within a certain tissue or organ. Electroencephalography (EEG) and in particular event-related potentials (ERPs) provide a possibility to non-invasively observe the living brain at work. EEG is a continuous recording of overall brain activity and is known to be able to detect abnormalities in the functioning of the brain. Since EEG recordings reflect the overall electrical activity of the brain, it is rather limited in providing insights into cognitive processes. ERPs on the other hand, allow insights into cognitive processes and are highly suited for addressing questions about the time course of cognitive processes. ERPs are retrieved from EEG signals by a process of event- (e.g. stimulus-) related averaging: averaging a large amount of trials cancels out noise and reveals consistent and therefore relevant brain activity aligned to an external event. A technique related to EEG is is magnetoencephalography (MEG). MEG makes use of the magnetic properties of electrical events associated with synaptic activity. MEG provides the same temporal resolution in the milliseconds (ms) range as EEG and allows the same process of event-related averaging (resulting in magnetic field potentials), but is superior in terms of localising the source of the signal. Positron emission tomography (PET) and functional magnetic resonance imaging (fMRI) are a further and different kind of functional imaging techniques. They detect changes in metabolism or blood flow in the brain while participants are engaged in a cognitive task. Unlike EEG and MEG, PET and fMRI do not measure neural events directly. Rather, they measure metabolic changes correlated with neural activity.

Among all these different new methods in empirical brain research, MRI and fMRI are likely to represent the most powerful anatomical and functional brain imaging techniques as they allow non-invasive, quick, reliable and repeated measurements of anatomical brain structure and task-related brain activity changes with high spatial and good temporal resolution (Goebel, 2007). These techniques will be elaborated on in the following section.

\section{MAGNETIC RESONANCE IMAGING (MRI)}

Since its development in the early 1980 's MRI has proven to be a very powerful tool for structural imaging. In direct comparison to other available imaging techniques, like CT, MRI uses a signal that is intrinsic to the tissue, and a signal contrast that is solely based on biochemical tissue properties. Since MRI operates on the radio frequency ( $r$ ) energy band and since most biological tissue is transparent at this energy level, the resolution quality does not decrease with tissue depth. Moreover, the radiation in MRI is completely nonionising and has no destructive effect on the tissue.

The physical principles underlying MRI are based on a phenomenon called nuclear magnetic resonance, which refers to the distinct behaviour of nuclei with odd numbers of protons that are exposed to a static magnetic field. The physical 
term of magnetic resonance refers to the reciprocity between the spins of protons and externally applied magnetic fields. Protons that are exposed to a strong static homogeneous magnetic field physically behave like small spinning bar magnets and orientate themselves parallel to this magnetic field by developing a net alignment of their spin axes along the direction of the applied magnetic field. The equilibrium state of the protons can be disturbed by applying a brief electromagnetic pulse with a radio frequency ( $r f$ ) exactly equivalent to the energy difference between the parallel (low energy) and antiparallel (high energy) protons aligned within the static magnetic field (the precessional frequency). This applied pulse of radio waves provides the energy to flip the spinning protons away from their equilibrium state into a different alignment angle that in turn depends on the duration of the applied radio frequency pulse. Usually the aspired alignment is 90 degrees to the alignment of the static field, because at this angle the observable output signal is at its maximum.

The phenomenon that occurs when a proton is exposed to an oscillating perturbation that has a frequency close to its natural frequency of oscillation is called resonance. The proton gains energy and resonates if the energy is delivered at exactly the same precessional frequency. This ability of the protons to absorb the energy provided by externally applied radio frequency pulses is called nuclear magnetic resonance. After the rf pulse is turned off, the protons return to their equilibrium state, which represents a lower-energy state, and in so doing release this energy as a signal in the form of radio waves. In physical terms, the nuclear spins of the protons reemit energy, creating coherent magnetisation in the transverse plane, which decays with time as a function of the spin system returning to its equilibrium state. The magnetisation induces a voltage as it passes through the respective coil. The magnitude of this induced voltage in the receiving coil changes over time depending on the different chemical and physical properties of the local tissue surrounding the protons, enabling the inference about the anatomical structure of the imaged tissue. These principles are the basis of the free induction decay (FID) signal in MRI. A second rf pulse with a duration that leads to a flip angle of 180 degrees can be applied after the initial rf pulse. This refocusing pulse reverses the dephasing of the spins and thus creates a so-called spin echo at a time that equals twice the time between the two applied rf pulses. Sequences using this technique are called gradient echo sequences and are more robust against possible static magnetic field inhomogeneity.

The frequency of the FID depends on the distinct magnetic field from spins in the different tissues, and thus enables an analysis of the voltage output based on these frequency differences, resulting in an image of the proton density at any point in space. The main contrast for this image generation is the density of protons, and since most protons are part of the $\mathrm{H}_{2} \mathrm{O}$ molecules, this contrast mainly represents the tissue water content. Nonetheless, there are two other contrast parameters that can be extracted in MRI. These contrast parameters are based on the fact that the rate with which protons return to a lower-energy level, their socalled relaxation, can be characteristically described by distinct time constants. There are two types of relaxation times important for MRI. One is called the longi- 
tudinal relaxation time ( $\mathrm{T} 1$ ), representing the time the spin system needs to recover to its thermal equilibrium, and the second one is called the transverse relaxation time (T2), representing the time needed by the excited spins to develop a phase incoherence before relaxing back to the equilibrium state. Both of these times depend on certain properties of the tissue surrounding the protons and thus represent contrast parameters that can be visualised in the obtained images as different image intensities. This technique allows the discrimination of different components of the tissue.

Although all of the described contrast parameters in MRI are very useful in imaging the anatomical structure of the brain tissue, none of them are affected by electrical activation in the neuronal structures or modulated by metabolic processes accompanying neuronal activity. The application of MRI for the imaging of functional processes in the brain thus requires a different approach, or rather a different signal contrast, to be imaged.

\section{FUNCTIONAL MAGNETIC RESONANCE IMAGING (FMRI)}

Functional magnetic resonance imaging (fMRI) utilises the technique of MRI in order to visualise brain signal dynamics that represent indirect measurements of neural activation. It measures the hemodynamic response (change in blood flow) related to neural activity in the brain or spinal cord. The general principle of blood-oxygenlevel dependent (BOLD) fMRI is based on the characteristic relationships between blood flow and oxygen consumption, which are both associated with neuronal activity. Neuronal activity results in regional cerebral blood flow that is uncoupled from the actual regional oxygen consumption and thus leads to a decrease in deoxyhaemoglobin. The idea of using BOLD signal is based on this observation.

Thus, an increase in neural activity initially leads to an increase in the metabolic rate of oxygen consumption and subsequently to an increase in the regional concentration of deoxyhemoglobin (because regional oxygen is absorbed). Due to the magnetic properties of deoxyhemoglobin which is paramagnetic, its presence distorts the magnetic field, resulting in a relative decrease of the BOLD fMRI signal (initial dip). However, with a certain delay, the increased metabolic rate of oxygen is compensated by a respective increase in the regional cerebral blood flow and blood supply to this region. Since this regional cerebral blood flow is uncoupled from the actual regional oxygen consumption and demand, it results in an oversupply of oxygenated haemoglobin, reversing the regional ratio between deoxyhemoglobin and oxyhemoglobin. Due to the magnetic properties of oxyhemoglobin, which is diamagnetic and thus does not distort the BOLD fMRI signal, the initial drop is followed by a delayed but larger increase of the BOLD signal. The signal is detectable by the MR scanner and is used as the indirect measurement of neural activity in fMRI. This method thus uses the blood oxygenation level as an endogenous contrast agent for the BOLD signal based fMRI, which enables the noninvasive observation of the hemodynamic changes associated with neuronal activity without the necessity of an exogenous contrast agent. 


\section{LIMITATIONS OF FUNCTIONAL IMAGING FOR THE INVESTIGATION OF BRAIN-BEHAVIOUR-RELATIONSHIPS}

The techniques of functional imaging are able to show an association between different behavioural or cognitive functions and patterns of neuronal activity on-line. Within strict and carefully designed functional imaging studies it seems reasonable to conclude with certain reasonability that the observed correlation between the applied behavioural or cognitive tasks and the measured brain activations can be attributed to a causal relationship. But a causal relationship between the observed changes in neuronal activation and the execution of a cognitive function is not necessarily true. In functional imaging, a relevant relation between the passively measured brain activity and the investigated cognitive functions always has to be assumed. This assumption, however, does not necessarily have to be valid. Many activation patterns seen in functional imaging may in fact represent a nonfunctional by-product of task execution, a mere epiphenomenon, or an activity that although specifically co-activated during task execution, does not represent a functionally necessary activation node for actual task performance. Moreover, a taskcorrelated increase in BOLD signal within a particular brain region might in fact also indicate that this region is not supporting successful task execution, but shows increased metabolic activity because it tries to inhibit the execution of this function.

In a similar vein, the activity could represent the attempt of this brain region to suppress other, competing functions in order to enable proper task execution. The metabolic imaging techniques can thus not distinguish the nature of the revealed activity changes, i.e., whether they are excitatory or inhibitatory in nature. Moreover, the fact that an area is active during the execution of a task does not provide any evidence for the fact that its activity is really needed. Therefore any concrete functional relationship between brain and behaviour remains speculative (Sack \& Linden, 2003). Furthermore, in the case of an identified distributed network of cortical activity during the execution of a complex cognitive task, functional imaging is not capable of distinguishing the various contributions of different brain areas within a complex task execution that consists of multiple components. In sum, the mere computation of a correlation between neuronal activity and certain stimulation paradigms or cognitive tasks does not allow any conclusions about the causal relationship or functional relevance of the measured neuronal activation patterns for the actual performance in these tasks.

All of the above mentioned limitations arise from the experimental designs employed in functional imaging. In these studies, brain activity is the dependent variable, uncontrollable by the experimenter. Functional imaging thus measures the changes in brain activity, depending on an experimental manipulation of task condition. The task itself is the independent variable and its influence on brain activity is measured. Functional neuroimaging can thus only state whether changing the task condition will result in changes in brain activity, but it cannot make any claims about whether the change in brain activity itself will cause a change in performing the different task conditions. 
Regarding causality, however, a brain region can only be considered functionally relevant for a certain behaviour or cognitive performance if changes in the activity of this region systematically result in a modulation of task performance. As a result, the experimental investigation of causal brain-behaviour-relationships would require a technique which can actually control and manipulate neural activity itself and investigate the consequence of this experimentally-induced neural activity change on perception and behaviour. Techniques that are capable of such controlled interferences with ongoing brain activity are referred to as brain interference techniques.

\section{BRAIN INTERFERENCE TECHNIQUES}

The class of brain interference techniques can be divided into invasive and noninvasive techniques. Cooling and microstimulation are invasive methods and therefore mainly used in animal studies. However, since higher cognition is unique to humans, research on higher cognition is in need of a suitable non-invasive technique. Transcranial magnetic stimulation (TMS) offers such a non-invasive brain interference approach.

\section{TRANSCRANIAL MAGNETIC STIMULATION (TMS)}

\section{HISTORY AND DEVELOPMENT OF TMS}

In 1831, Faraday discovered the principle of mutual induction which addresses the physical phenomenon that electrical fields can be converted into magnetic fields, and magnetic fields can be converted into electrical energy. Based on this principle, Faraday described the so-called electromagnetic induction, which refers to the possibility to produce a current in a conductive medium by either moving the conductive object through a static magnetic field, or by placing the conductive object into a time-varying magnetic field.

The technique of TMS depends on these basic principles of mutual and electromagnetic induction. The first systematic experiments with magnetic stimulation were conducted by d'Arsonval in 1896 (D'Arsonval, 1896). He was able to produce phosphenes (flickering lights in the visual field), vertigo, and even syncope after placing a participant's head inside a powerful magnetic coil. Nonetheless, several scientists speculated later that these phosphenes were mere magnetophosphenes, the result of a direct stimulation of the retina rather than of the visual cortex (Barlow, 1947; Dunlap, 1911; Magnusson, 1911; Thompson, 1910; Walsh, 1946). In 1902 , Beer replicated this finding and reported that phosphenes could also be produced by applying an oscillating strong magnetic field to the head (Beer, 1902).

Since that time several researchers have attempted to construct different magnetic stimulation devices in order to produce and investigate phosphenes systematically (Dunlap, 1911; Magnusson, 1911; Thompson, 1910). Unfortunately these devices were very slow to recharge and thus very restricted in their intensity 
and frequency capabilities. Nonetheless, the development of more precise and more powerful magnetic stimulators continued and still continues today.

Magnetic stimulation of isolated nerves was accomplished only several decades later, first in the frog by Kolin and colleagues in 1959 (Kolin, 1959) and then in the human peripheral nerve by Bickford and Fremming (1965) who, in 1965, demonstrated the non-invasive magnetic stimulation of facial nerves. In 1982, Polson and colleagues (Polson, Barker, \& Freeston, 1982) produced and described a magnetic stimulator for peripheral nerve stimulation. Using pulses of 2 ms duration they recorded the first motor evoked potentials (MEPs).

An important landmark and maybe the beginning of modern TMS was the development of the first TMS device by Barker in 1985 by (Barker, 1985). By stimulating the human motor cortex and inducing the respective motor contractions they measured the connectivity and excitability of the motor cortex in healthy participants. Moreover Barker (1985) performed the first clinical examinations by comparing conduction times of motor response in healthy participants and patients with different neurological diseases (Barker, 1985; Barker, Freeston, Jabinous, \& Jarratt, 1986).

\section{BASIC PHYSICAL PRINCIPLES OF TMS}

Transcranial magnetic stimulation (TMS) is based on the aforementioned principles of electromagnetic induction. A large and brief pulse of current is discharged into an electromagnetic coil held above a participant's head. This current produces a timevarying magnetic field perpendicular to the current, lasting for approximately 100 to 200 microseconds. The magnetic field passes into the tissue and induces a perpendicular electric field. The strength of the induced electric field mainly depends on the rate of change of the magnetic field. Due to the electrical conductivity of the living tissue, the electric field leads to an electrical current in the cortex parallel but opposite in direction to the current in the coil (Lenz's law), and subsequently to a depolarisation of the underlying neurons (Hallett, 2000).

Physically the rate of change of the electric current in the coil determines the strength of the induced electrical field. Therefore the electrical field has its maximum at the instant of the switching of the electrical current since this point marks the maximum rate of change of the current. With increasing current strength this rate of change decreases and consequently the induced electrical field strength decreases, reaching zero at the current's maximum value. With subsequent current decrease, the rate of change increases exponentially to a new maximum, creating another peak in the induced electrical field. Consequently, the induced electrical field has two pulses, the first approximately $100 \mu$ s long, and the second about 150 $\mu$ s long and slightly less intense.

\section{TMS HARDWARE}

Any TMS device consists of a bank of capacitors capable of producing high discharge currents, and an electromagnetic stimulating coil to apply magnetic pulses of up to 4 Tesla. The high and rapidly changing currents are discharged into the coil, thereby creating a strong and time-varying magnetic field (pulse) that can 
reach its peak in less than $250 \mu \mathrm{s}$. This time varying magnetic field induces an electric current in the neuronal tissue underneath the coil, and thus induces action potentials when applied at proper intensities. Different geometric shapes of the electromagnetic coils allow for different stimulation characteristics, including focality, depth of penetration, and strength of the induced activity changes.

For example, in the so-called circular coil, low-resistance copper is wound in one or several turns into a ring-shaped configuration. This coil type has no single magnetic field focus but a maximum current in the entire outer winding, forming a ring shaped magnetic field around the coil. Therefore, the site of stimulation in a circular coil is not well defined. Inside a figure-of-eight coil are two ring-shaped coils mounted next to each other and coated by the characteristic butterfly-shaped coil mantle. Importantly, the current inside each of these two coil windings circulates in opposite directions, causing the two respective magnetic fields of these two coil loops to add up at the coils ' intersection. This enables more focal magnetic brain stimulation as compared to other coil geometries, making it generally speaking more suitable for research in cognitive neuroscience, e.g., in the context of functional mapping of the brain or causal brain-behaviour relationships.

\section{CHRONOMETRIC VERSUS REPETITIVE TMS}

TMS can be applied one stimulus at a time, single-pulse TMS, in pairs of stimuli separated by a variable interval, paired-pulse TMS, or in multiple pairs of pulses, ranging from triple-pulse up to quintuple-pulse TMS. Importantly, all of these ways of applications are usually linked to the onset of an external event such as taskonset, and therefore capable of revealing information about the chronometry of a cognitive process. We therefore can refer to these approaches as chronometric or event-related TMS, or in other words, protocols used to study causal chronometry in brain-behaviour relations. In contrast, TMS can also be applied in a repetitive manner (rTMS). Today, we distinguish between conventional and patterned protocols of repetitive stimulation (Rossi, Hallett, Rossini, \& Pascual-Leone, 2009). In conventional protocols, single TMS pulses are repeated and applied in a regular rhythm. Here, one can once again make a distinction between low-frequency rTMS (stimulation frequency of $1 \mathrm{~Hz}$ or less) and high-frequency rTMS (stimulation frequency of more than $1 \mathrm{~Hz}$ ). With newly developed TMS stimulators, the maximum stimulation frequency that can be reached lies at $100 \mathrm{~Hz}$. Patterned rTMS refers to repetitive application of short rTMS bursts at a high inner frequency interleaved by short pauses of no stimulation. Only recently, a new protocol was developed called theta burst stimulation (TBS). In TBS protocols, short bursts of $50 \mathrm{~Hz}$ rTMS are repeated at a rate in the theta range $(5 \mathrm{~Hz})$ as a continuous (CTBS) or intermittent (iTBS) train (Di Lazzaro, 2008; Huang, Edwards, Rounis, Bhatia, \& Rothwell, 2005).

The important feature of both conventional and patterned TMS is that it is capable of modulating the excitability of the stimulated area even beyond the duration of the TMS application itself. The after effects of TBS were found to be significantly longer-lasting compared to conventional rTMS (Huang, Edwards, Rounis, Bhatia, \& Rothwell, 2005) with shorter stimulation time needed. Patterned rTMS protocols are starting to play a bigger role in brain stimulation. Both $1 \mathrm{~Hz}$ rTMS and 
CTBS are consistently found to produce lasting inhibitory after effects. Highfrequency rTMS and iTBS on the other hand induced lasting facilitory after effects on motor corticospinal output in healthy participants. The precise neuronal mechanisms of effects that last beyond the duration of the TMS application itself are not fully known yet. However, similarities to neurophysiological electrical stimulation studies in animals are apparent. In these studies direct high-frequency electrical stimulation resulted in long term increases of intersynaptic transmission efficiency, termed long-term potentiation (LTP), while low-frequency electrical stimulation led to a long-term decrease of intersynaptic transmission efficiency, called long-term depression (LTD) (Artola, Brocher, \& Singer, 1990; Silkis, Rapoport, \& Veber, 1994; Stanton \& Sejnowski, 1989). Other mechanisms such as shifts in network excitability, activation of feedback loops and activity-dependent metaplasticity (Gentner, Wankerl, Reinsberger, Zeller, \& Classen, 2008; Iezzi et al., 2008) might also account for the long-lasting effects.

In sum, the number of repetitive stimuli per second, the stimulation frequency, as well as the stimulus intensity, the duration of the stimulation train, the inter-train interval, and finally the total number of trains and total number of stimuli represent the stimulation parameters of TMS which may all be combined in different ways to produce the desired neuronal effects at the targeted brain site.

\section{THE PRINCIPLE OF TMS EFFECTS ON NEURONAL TISSUE}

The magnetic stimulation indirectly creates a transmembrane potential by moving a charge across the cellular membrane which can lead to membrane depolarisation and hence to an action potential of the respective axon. An electric field parallel to the axon causes current flow inside and outside, but does not lead to a change of the transmembrane potential of the axon. But if this current inside the axon changes along the nerve, a current similar in strength to this change has to pass through the membrane of the axon leading to a respective change of the transmembrane potential. If this membrane potential passes a crucial threshold, it can result in an action potential of the axon.

The temporal-spatial characteristics of the magnetic pulse depend on the physical parameters of the magnetic field such as rise time and spatial field distribution, which underlie the electrical characteristics of the stimulator and the coil. The temporal-spatial characteristics of the induced electric field, and thus the actually stimulated area, depend on several additional factors such as scalp shape, scalp-cortex-distance, coil shape, stimulation intensity and monophasic vs. biphasic pulse application. The magnetic field strength decreases logarithmically with distance from the coil, which limits the area of depolarisation. Generally speaking, the spatial distribution of the cortical area affected by current TMS devices has approximately a diameter of $3 \mathrm{~cm}$ long, $2 \mathrm{~cm}$ wide and $3 \mathrm{~cm}$ deep, measured from the centre of the coil (Rudiak \& Marg, 1994). However, several blood flow and imaging studies demonstrated that TMS may affect remote cortical and subcortical areas via transsynaptical connections (Bestmann et al., 2008; Paus et al., 1997; Ruff et al., 2009; Ruff, Driver, \& Bestmann, 2009; Sack et al., 2007). 


\section{SAFETY OF TMS}

For the safety of TMS, a variety of effects must be considered. Generally, due to the application of strong magnetic pulses, TMS may lead to heating of any metallic objects near the stimulation sites. Furthermore, the magnetic field pulse generated by the TMS coil exerts forces on ferromagnetic objectives and non-ferromagnetic conductors. Thus, TMS can theoretically result in forces on head implants that could potentially displace them. Therefore the same precautions should be taken as in the case of clinical magnetic resonance imaging. This includes the general absence of magnetic objects like pacemakers, medication pumps, cochlear prostheses, or other implanted metallic objects, magnetic media and watches.

Possible side effects of TMS include uncomfortable sensation during stimulation, local pain at stimulation site, neck pain, toothache, headaches, muscle twitches, transient hearing changes, transient cognitive/neuropsychological changes, syncope, and seizure induction. Headaches after TMS sessions can easily be treated with common pain medication. Transient hearing changes can be prevented by participants wearing appropriate hearing protection during the entire stimulation session. The transient change in cognitive performance is often a desired change in the cognitive domain, since we are interested in how stimulating a certain site affects cognition. Thus a desired form of cognitive change is a participant performing better or worse in a given task (percentage correct responses, reaction times, etc.). These changes are often only small and temporary. Muscle twitches are a common side effect of TMS when stimulating frontal or temporal sites. They can be an uncomfortable sensation but are not harmful.

The most severe acute adverse effect of TMS is the induction of a seizure. However, considering the large number of participants and patients tested with TMS to date, the risk of inducing seizures with TMS is extremely low. Seizures are caused by hypersynchronised discharges of groups of neurons in the grey matter, mainly due to an imbalance between inhibitory and excitatory synaptic activity, in favour of the latter (Rossi, Hallett, Rossini, \& Pascual-Leone, 2009). A total of 16 cases of induced seizures due to both single-pulse as well as repetitive TMS have been reported thus far. Based on reports and our knowledge about potential hazards for TMS, there are guidelines over who should and who should not receive TMS. Since we work in a non-clinical environment and are mainly interested in the functioning of the normal healthy brain, we use the strictest inclusion and exclusion criteria possible. People who should generally be excluded from TMS studies are those with a (family) history of epilepsy, a history of head trauma, history of drug abuse, heart diseases, and pregnancy. Furthermore, any participant receiving medication that might lower the participant's seizure threshold should also be excluded. Prior to every experimental session, we also make sure that the participant had enough sleep and did not consume any alcohol or drugs the night before.

In addition to strict exclusion and inclusion criteria, several safety guidelines concerning the stimulation intensity, stimulation frequency etc have been published (Chen et al., 1997; Grossheinrich et al., 2009; Rossi, Hallett, Rossini, \& PascualLeone, 2009; Wassermann, 1998). 


\section{TMS AS A TOOL TO STUDY HIGHER COGNITION}

As detailed above, TMS can be applied in a chronometric, event-related manner as well as in a repetitive manner. Both manners provide excellent tools for the study of cognition. Applying TMS in a chronometric manner, appropriately delivered in time and space, the function of a cognitive process is disturbed online. Applying TMS repetitively results in an offline, longer-lasting effect. Those two effects enable the study of two aspects of the contribution of a given cortical region to a specific behaviour: 'what does it do?' and 'when does it do it?'. We are particularly interested in higher cognitive functions. This means that the target sites of the areas of interest are often silent (higher-order areas). The higher order areas do not have a directly observable effect of stimulation, which raises the question as to where to exactly position the coil. When stimulating a non-silent area, like the motor cortex, we immediately know whether or not we are on the right spot with the coil, since we either see a reaction in, for example, a hand muscle, or not. Since this is not possible for silent areas, other options for coil placement were found. The most advanced approach of positioning the TMS coil over silent brain areas in the study of cognition is TMS Neuronavigation.

\section{NEURONAVIGATION FOR TMS}

The precise and accurate positioning of the TMS coil over the target site represents one of the most challenging aspects of experimental procedure. Successful neuronavigation in TMS involves careful consideration of interindividual anatomical variability, in particular in case of target areas with large inter-subject variability such as the prefrontal cortex or along the intraparietal sulcus (Sparing, Hesse, \& Fink, 2010). There are various approaches on how to place a TMS coil. As elaborated above, a commonly used positioning strategy draws upon a reference point at which TMS directly elicits an objective response (non-silent areas). Another strategy makes use of the 10-20 EEG electrode system and assumes a correlation between scalp locations and underlying brain structures. The function-guided approach is based on prior knowledge of a TMS effect on a certain behavioural task. If this effect is known, the performance on this task can function as a probe to position the coil for the succeeding investigation of another task. To best account for interindividual anatomical differences, various image-guided stereotaxic neuronavigation systems have been developed. Neuronavigation systems use the participant's or patient's MRI for navigation via a participant - reconstructed image coregistration procedure based on facial landmarks. The systems thus allow for an online individual navigation of a TMS coil above a specific anatomical area of the brain, as well as an imaging-guided navigation of the TMS coil to functionally defined brain regions-of-interest. In our lab, we make use of the BrainVoyager TMS Neuronavigator (BrainInnovation, Maastricht, The Netherlands) (Sack, Kohler, Linden, Goebel, \& Muckli, 2006). This neuronavigation system consists of several miniature ultrasound transmitters which are attached to a participant's head, as well as the TMS coil. The ultrasound markers continuously transmit ultrasonic pulses to a receiving sensor 
device. The measurement of the relative spatial position of these transmitters in 3D space is based on travel time of the transmitted ultrasonic pulses to three microphones of the receiving sensor device. Local spatial coordinate systems are created by linking the relative raw spatial position of the ultrasound senders to a set of fixed additional landmarks on the participant's head. The specification of these fixed landmarks is achieved via a digitising pen that also hosts two transmitting ultrasound markers in order to measure its relative position in 3-D space. The three anatomical landmarks we used to define the local coordinate system were the nasion and the two incisurae intertragicae. The neuronavigation system then provides topographic information of the head ultrasound transmitters relative to a participant-based coordinate frame. Similarly, the TMS coil also hosts a set of ultrasound transmitters whose relative spatial positions are linked to fixed landmarks specified on the coil in order to calculate another local coordinate system. After having defined the local spatial coordinate system for the participant's head and the TMS coil in real 3-D space, these coordinate systems have to be co-registered with the coordinate system of the MR space. For TMS-MRI co-registration, the same landmarks digitised on the participant's head are specified on the head reconstruction of the anatomical data from the MR sequence. After the landmarks specified on the real head have been co-registered with those on the reconstructed head, events occurring around the head of the participant in real space are registered online and visualised in real-time at correct positions relative to the participant's anatomical reconstruction of the brain. The TMS coil can now be neuronavigated to a specific anatomical area of each participant. Even when using such a neuronavigation system, the method on how to determine the exact stimulation target site for TMS can still be based on different positioning criteria and the question which of those positioning criteria is optimal in cognitive TMS studies represents an empirical question (see Chapter 2).

\section{STIMULATING LANGUAGE-RELATED AREAS}

An example of a higher cognitive skill involving silent brain areas is language. Language processing is one of the most complex cognitive skills. The question rises whether we can go as far as to stimulate such a complex process likely involving a highly distributed brain network with TMS. Pioneers in the stimulation of languagerelated areas were not cognitive neuroscientists, but rather neurosurgeons, namely Wilder Penfield and George Ojemann. They used direct cortical stimulation in awake neurosurgical patients to probe language processing (Ojemann, 1979; Penfield \& Jasper, 1954). Ojemann was able to show that applying a direct current to a focal brain region can selectively disrupt linguistic processes. As exciting as the findings were, the invasive nature of Ojemann's research did not allow for extensive testing of language functions in healthy individuals. The introduction of TMS however lead to new possibilities. Pascual-Leone and colleagues (Pascual-Leone, Gates, \& Dhuna, 1991) were the first to study language with TMS. They induced speech arrest in pre-surgical epilepsy patients in order to determine whether TMS could be used as a non-invasive alternative to intracarotid amorbarbital testing. Others were also 
able to show that high-frequency rTMS can produce transient speech arrests (Epstein, 1999; Epstein et al., 1996; Jennum, Friberg, Fuglsang-Frederiksen, \& Dam, 1994; Michelucci et al., 1994). Epstein et al.(1999) revealed that rTMS to the left inferior frontal area led to an arrest of spontaneous speech and reading aloud, while the general generation of language like writing, comprehension, singing etc. remained unaffected. Following these pioneering findings, several studies have aimed at using TMS in order to bring to light the functional role of Broca's area during language processing and production (Andoh et al., 2006; Devlin, Matthews, \& Rushworth, 2003; Mottaghy et al., 1999; Mottaghy, Sparing, \& Topper, 2006; Naeser et al., 2005; Nixon, Lazarova, Hodinott-Hill, Gough, \& Passingham, 2004; Sakai, Noguchi, Takeuchi, \& Watanabe, 2002; Shapiro, Pascual-Leone, Mottaghy, Gangitano, \& Caramazza, 2001). In a similar vein, Wernicke's area has been targeted by TMS (Andoh et al., 2006; Mottaghy et al., 1999; Mottaghy, Sparing, \& Topper, 2006; Töpper, Mottaghy, Brugmann, Noth, \& Huber, 1998) in order to unravel its functional contribution to language processing in healthy human volunteers.

These brain interference studies have contributed to the understanding of functional relevance of language-related brain areas to the behavioural execution of various linguistic tasks. However, only very few of these TMS studies employed the advantage of event-related TMS to track the exact time point of functional activation during language production. Moreover, most previous studies neglected the high inter-individual variance in the anatomy of participant's brains and applied TMS to sites that were defined by anatomical landmarks of the skull or standardised group coordinates, instead of making use of individual anatomical brain mapping. This PhD thesis aims at directly addressing all of these shortcomings and to develop and evaluate an optimised approach of targeting the silent brain areas in cognitive TMS studies. We directly apply these findings, extending the boundaries of TMS application in high-level cognition, by studying neural network accounts, functional relevance, and causal chronometry, of several brain regions in the domains of language production, and even the complex human practice of deception.

\section{OUTLINE OF THE THESIS}

The study "Optimising functional accuracy of TMS in cognitive studies: A comparison of methods" presented in Chapter $\mathbf{2}$ is a methodological study, systematically comparing four commonly used TMS coil positioning approaches for studying structure-function relationships in higher cognition. Specifically, it investigates the behavioural impact of right parietal TMS during a number comparison task, while basing TMS localisation either on 1) individual fMRI-guided TMS Neuronavigation, 2) individual MRI-guided TMS Neuronavigation, 3) navigating based on group functional Talairach Coordinates, or 4) an anatomical landmark approach using the 1020 EEG electrode position P4. Based on the results of this study, we used an optimised functional accuracy in coil positioning for our study "The temporal characteristics of functional activation in Broca's area during overt picture naming" described in Chapter 3. In this study, we investigate both the functional role as well as the 
precise temporal involvement of Broca's area during language production, as taking place during picture naming. We applied real and sham online event-related TMS to Broca's area at five different time points after picture presentation, aiming to cover the complete language production process. Intrigued by our findings, we performed another study entitled "Speaking of which: Dissecting the neurocognitive network of language production". Here, we not only stimulated Broca's area, but also two other prominent language-related areas, namely Wernicke's area and the middletemporal-gyrus (MTG). Specifically, we probed the behavioural relevance of all three brain areas for successful language production by directly interfering with their local neural activity changes online, i.e., at various time points during overt picture naming, using MRI-guided chronometric TMS. This enabled us to test whether neural activity in these regions is functionally relevant for picture naming and to chart the exact relative time point at which this neural activity is critical. This work is outlined in Chapter 4.

Chapter 5 describes a study covering another aspect of human higher cognition, namely deception. To examine the role of the right inferior frontal sulcus (IFS) in deception, we experimentally manipulated IFS activity using a relatively new TMS paradigm, namely theta burst stimulation, and measured offline the impact of this TMS-induced IFS disruption on accuracy and reaction times during a behaviourallycontrolled deception paradigm ("The functional role of inferior frontal sulcus in deception: A neuronavigated theta-burst transcranial magnetic stimulation study"). 


\section{REFERENCES}

Andoh, J., Artiges, E., Pallier, C., Riviere, D., Mangin, J. F., Cachia, A., et al. (2006). Modulation of language areas with functional MR image-guided magnetic stimulation. Neuroimage., 29(2), 619627. Epub 2005 Sep 2015.

Artola, A., Brocher, S., \& Singer, W. (1990). Different voltage-dependent thresholds for inducing longterm depression and long-term potentiation in slices of rat visual cortex. Nature, 347(6288), 69-72.

Barker, A. T. (1985). Non-invasive magnetic stimulation of human motor cortex. The Lancet, 1(8437), 1106-1106.

Barker, A. T., Freeston, I. L., Jabinous, R., \& Jarratt, J. A. (1986). Clinical evaluation of conduction time measurements in central motor pathways using magnetic stimulation of human brain. The Lancet, 1(8493), 1325-1326.

Barlow, H. B. (1947). Visual sensations aroused by magnetic fields. American Journal of Physiology, $148,372-372$.

Beer, B. (1902). Über das Auftreten einer objektiven Lichtempfindung in magnetischen Felde. Klinische Wochenzeitschrift, 15, 108-109.

Bestmann, S., Swayne, O., Blankenburg, F., Ruff, C. C., Haggard, P., Weiskopf, N., et al. (2008). Dorsal premotor cortex exerts state-dependent causal influences on activity in contralateral primary motor and dorsal premotor cortex. Cerebral Cortex, 18(6), 1281-1291.

Bickford, R., \& Fremming, B. (1965). Neural stimulation by pulsed magnetic fields in animals and man. Paper presented at the Conference Name|. Retrieved Access Date|. from URL|.

Chen, R., Gerloff, C., Classen, J., Wassermann, E. M., Hallett, M., \& Cohen, L. G. (1997). Safety of different inter-train intervals for repetitive transcranial magnetic stimulation and recommendations for safe ranges of stimulation parameters. Electroencephalogry and Clinical Neurophysiology, 105(6), 415-421.

D'Arsonval, A. (1896). Dispositifs pour la mesure des courants alternatifs de toutes fréquences. Comptes Rendus des Séances et Mémoires de la Société de Biologie, 3, 450-457.

Devlin, J. T., Matthews, P. M., \& Rushworth, M. F. (2003). Semantic processing in the left inferior prefrontal cortex: a combined functional magnetic resonance imaging and transcranial magnetic stimulation study. Journal of Cognitive Neuroscience, 15(1), 71-84.

Di Lazzaro, V. (2008). The physiological basis of the effects of intermittent theta burst stimulation of the human motor cortex. The Journal of physiology, 586(16), 3871-3871.

Dunlap, K. (1911). Visual Sensations from the Alternating Magnetic Field. Science, 33(837), 68-71.

Epstein, C. M. (1999). Language and TMS/rTMS. Electroencephalography and Clinical Neurophysiology. Supplement, 51, 325-333.

Epstein, C. M., Lah, J. J., Meador, K., Weissman, J. D., Gaitan, L. E., \& Dihenia, B. (1996). Optimum stimulus parameters for lateralized suppression of speech with magnetic brain stimulation. Neurology, 47(6), 1590-1593.

Gentner, R., Wankerl, K., Reinsberger, C., Zeller, D., \& Classen, J. (2008). Depression of human corticospinal excitability induced by magnetic theta-burst stimulation: evidence of rapid polarityreversing metaplasticity. Cerebral Cortex, 18(9), 2046-2053.

Goebel, R. (2007). Localization of brain activity using functional magnetic resonance imaging. In C. Stippich (Ed.), Clinical Functional MRI - Presurgical Functional Neuroimaging. Heidelberg: Springer.

Grossheinrich, N., Rau, A., Pogarell, O., Hennig-Fast, K., Reinl, M., Karch, S., et al. (2009). Theta burst stimulation of the prefrontal cortex: safety and impact on cognition, mood, and resting electroencephalogram. Biological Psychiatry, 65(9), 778-784.

Hallett, M. (2000). Transcranial magnetic stimulation and the human brain. Nature, 406(6792), 147150.

Huang, Y.-Z., Edwards, M. J., Rounis, E., Bhatia, K. P., \& Rothwell, J. C. (2005). Theta Burst Stimulation of the Human Motor Cortex. Neuron, 45(2), 201-206.

Iezzi, E., Conte, A., Suppa, A., Agostino, R., Dinapoli, L., Scontrini, A., et al. (2008). Phasic voluntary movements reverse the aftereffects of subsequent theta-burst stimulation in humans. Journal of Neurophysiology, 100(4), 2070-2076.

Jennum, P., Friberg, L., Fuglsang-Frederiksen, A., \& Dam, M. (1994). Speech localization using repetitive transcranial magnetic stimulation. Neurology, 44(2), 269-273. 
Kolin, A. (1959). Miniaturization of the electromagnetic blood flow meter and its use for the recording of circulatory responses of conscious animals to sensory stimuli. Proceedings of the National Academy of Sciences of the United States of America, 45(8), 1312-1312.

Magnusson, C. E. (1911). Visual sensations produced by changes in the strength of a magnetic field. American journal of physiology, 29, 124-124.

Michelucci, R., Valzania, F., Passarelli, D., Santangelo, M., Rizzi, R., Buzzi, A. M., et al. (1994). Rapidrate transcranial magnetic stimulation and hemispheric language dominance: usefulness and safety in epilepsy. Neurology, 44(9), 1697-1700.

Mottaghy, F. M., Hungs, M., Brugmann, M., Sparing, R., Boroojerdi, B., Foltys, H., et al. (1999). Facilitation of picture naming after repetitive transcranial magnetic stimulation. Neurology, 53(8), 1806-1812.

Mottaghy, F. M., Sparing, R., \& Topper, R. (2006). Enhancing picture naming with transcranial magnetic stimulation. Behavioral Neurology, 17(3-4), 177-186.

Naeser, M. A., Martin, P. I., Nicholas, M., Baker, E. H., Seekins, H., Kobayashi, M., et al. (2005). Improved picture naming in chronic aphasia after TMS to part of right Broca's area: an openprotocol study. Brain and Language, 93(1), 95-105.

Nixon, P., Lazarova, J., Hodinott-Hill, I., Gough, P., \& Passingham, R. (2004). The inferior frontal gyrus and phonological processing: an investigation using rTMS. Journal of Cognitive Neuroscience, 16(2), 289-300.

Ojemann, G. A. (1979). Individual variability in cortical localization of language. Journal of Neurosurgery, 50(2), 164-169.

Pascual-Leone, A., Gates, J. R., \& Dhuna, A. (1991). Induction of speech arrest and counting errors with rapid-rate transcranial magnetic stimulation. Neurology, 41(5), 697-702.

Paus, T., Jech, R., Thompson, C. J., Comeau, R., Peters, T., \& Evans, A. C. (1997). Transcranial Magnetic Stimulation during Positron Emission Tomography: A New Method for Studying Connectivity of the Human Cerebral Cortex. Journal of Neuroscience, 17(9), 3178-3184.

Penfield, W., \& Jasper, H. H. (1954). Epilepsy and the functional anatomy of the human brain. Boston: Little, Brown, and Co.

Polson, M., Barker, A., \& Freeston, I. (1982). Stimulation of nerve trunks with time-varying magnetic fields. Medical and Biological Engineering and Computing, 20(2), 243-244.

Rossi, S., Hallett, M., Rossini, P. M., \& Pascual-Leone, A. (2009). Safety, ethical considerations, and application guidelines for the use of transcranial magnetic stimulation in clinical practice and research. Clinical Neurophysiology, 120(12), 2008-2039.

Rudiak, D., \& Marg, E. (1994). Finding the depth of magnetic brain stimulation: a re-evaluation. Electroencephalography and Clinincal Neurophysiology, 93(5), 358-371.

Ruff, C. C., Blankenburg, F., Bjoertomt, O., Bestmann, S., Weiskopf, N., \& Driver, J. (2009). Hemispheric differences in frontal and parietal influences on human occipital cortex: direct confirmation with concurrent TMS-fMRI. Journal of Cognitive Neuroscience, 21(6), 1146-1161.

Ruff, C. C., Driver, J., \& Bestmann, S. (2009). Combining TMS and fMRI: from 'virtual lesions' to functional-network accounts of cognition. Cortex, 45(9), 1043-1049.

Sack, A. T., Kohler, A., Bestmann, S., Linden, D. E., Dechent, P., Goebel, R., et al. (2007). Imaging the brain activity changes underlying impaired visuospatial judgments: simultaneous FMRI, TMS, and behavioral studies. Cerebral Cortex, 17(12), 2841-2852.

Sack, A. T., Kohler, A., Linden, D. E. J., Goebel, R., \& Muckli, L. (2006). The temporal characteristics of motion processing in hMT/V5+: Combining fMRI and neuronavigated TMS. NeuroImage, 29(4), 1326-1335.

Sack, A. T., \& Linden, D. E. (2003). Combining transcranial magnetic stimulation and functional imaging in cognitive brain research: possibilities and limitations. Brain Research. Cognitive Brain Research, 43(1), 41-56.

Sakai, K. L., Noguchi, Y., Takeuchi, T., \& Watanabe, E. (2002). Selective priming of syntactic processing by event-related transcranial magnetic stimulation of Broca's area. Neuron, 35(6), 1177-1182.

Shapiro, K. A., Pascual-Leone, A., Mottaghy, F. M., Gangitano, M., \& Caramazza, A. (2001). Grammatical distinctions in the left frontal cortex. Journal of Cognitive Neuroscience, 13(6), 713720. 
Silkis, I. G., Rapoport, S. S., \& Veber, N. V. (1994). Long-term posttetanic changes of the neuronal reactions in microareas of the cat's motor cortex. Žurnal vysšej nervnoj deâtel'nosti im. I.P. Pavlova, 44, 124-134.

Sparing, R., Hesse, M. D., \& Fink, G. R. (2010). Neuronavigation for transcranial magnetic stimulation (TMS): where we are and where we are going. Cortex, 46(1), 118-120.

Stanton, P. K., \& Sejnowski, T. J. (1989). Associative long-term depression in the hippocampus induced by hebbian covariance. Nature, 339(6221), 215-218.

Thompson, S. P. (1910). A Physiological Effect of an Alternating Magnetic Field. Proceedings of the Royal Society of London. Series B, Containing Papers of a Biological Character, 82(557), 396-398.

Töpper, R., Mottaghy, F. M., Brugmann, M., Noth, J., \& Huber, W. (1998). Facilitation of picture naming by focal transcranial magnetic stimulation of Wernicke's area. Experimental Brain Research, 121(4), 371-378.

Walsh, P. (1946). Magnetic stimulation of the human retina. Federation Proceedings, 5, 109-110.

Wassermann, E. M. (1998). Risk and safety of repetitive transcranial magnetic stimulation: report and suggested guidelines from the International Workshop on the Safety of Repetitive Transcranial Magnetic Stimulation, June 5-7, 1996. Electroencephalography and Clinical Neurophysiology, $108(1), 1-16$. 



\section{CHAPTER 2}

\section{OPTIMISING FUNCTIONALACCURACY OF TMS IN COGNITIVE STUDIES: A COMPARISON OF METHODS ${ }^{1}$}

1: Based on: Sack, A.T., Cohen Kadosh, R., Schuhmann, T., Moerel, M., Walsh, V., \& Goebel, R. (2008). Optimizing functional accuracy of TMS in cognitive studies: A comparison of methods. Journal of Cognitive Neuroscience, 22, 207-221. 


\begin{abstract}
Transcranial Magnetic Stimulation (TMS) is a tool for inducing transient disruptions of neural activity non-invasively in conscious human volunteers. In recent years, the investigative domain of TMS has expanded and now encompasses causal structure-function relationships across the whole gamut of cognitive functions and associated cortical brain regions. Consequently, the importance of how to determine the target stimulation site has increased and a number of alternative methods have emerged. Comparison across studies is precluded because different studies necessarily use different tasks, sites, TMS conditions and have different goals. Here, therefore, we systematically compare four commonly used TMS coil positioning approaches by using them to induce behavioural change in a single cognitive study. Specifically, we investigated the behavioural impact of right parietal TMS during a number comparison task, while basing TMS localisation either on i) individual fMRIguided TMS Neuronavigation, ii) individual MRI-guided TMS Neuronavigation, iii) group functional Talairach Coordinates, or iv) 10-20 EEG position P4. We quantified the exact behavioural effects induced by TMS using each approach, calculated the standardised experimental effect sizes, and conducted a statistical power analysis in order to calculate the optimal sample size required to reveal statistical significance. Our findings revealed a systematic difference between the four approaches, with the individual fMRI-guided TMS Neuronavigation yielding the strongest and the P4 stimulation approach yielding the smallest behavioural effect size. Accordingly, power analyses revealed that while in the fMRI-guided neuronavigation approach five participants were sufficient to reveal a significant behavioural effect, the number of necessary participants increased to $n=9$ when employing MRI-guided neuronavigation, to $\mathrm{n}=13$ in case of TMS based on group Talairach coordinates, and to $n=47$ when applying TMS over P4. We discuss these graded effect size differences in light of the revealed inter-individual variances in the actual target stimulation site within and between approaches.
\end{abstract}




\section{INTRODUCTION}

Transcranial Magnetic Stimulation (TMS) allows controlled manipulation of brain activity, with a quantifiable impact on behaviour or cognition (Hallett, 2000; Pascual-Leone, Bartres-Faz, \& Keenan, 1999; Pascual-Leone, Walsh, \& Rothwell, 2000; Sack, 2006), and is now a well established tool for inducing transient disruptions of neural activity non-invasively in conscious human volunteers (Di Lazzaro et al., 2004; Pascual-Leone, Walsh, \& Rothwell, 2000; Robertson, Théoret, \& PascualLeone, 2003; Walsh \& Pascual-Leone, 2003). In recent years, TMS has expanded its investigative domain which has brought to the forefront questions of how to optimally target stimulation sites in individual participants, and to establish optimal and sufficient methods. Some areas, when stimulated, have a signature output: TMS over motor cortex causes a twitch (Ziemann, 2004), and TMS over visual cortex can induce phosphenes (Epstein, Verson, \& Zangaladze, 1996; Epstein \& Zangaladze, 1996; Stewart, Battelli, Walsh, \& Cowey, 1999). But many of the areas of interest in cognitive TMS studies, for example, the posterior parietal cortex, occipital face area, extrastriate body area, prefrontal cortex, inferior frontal gyrus, etc., are behaviourally silent (Penfield, 1958) and do not produce an immediately observable response. Thus localisation of target stimulation sites and positioning of the TMS coil presents a problem.

One common approach of localising the TMS target stimulation site of "silent" brain areas is to place the coil according to the international 10-20 electrode scalp positioning system (Jasper, 1958). Although this method may be sufficient for some purposes it does not take into account inter-individual differences in the functional architecture of the brain or differences in the correspondence between scalp landmarks and underlying brain anatomy (Herwig, Satrapi, \& Schönfeldt-Lecuona, 2003; Okamoto et al., 2004). With the introduction of frameless stereotaxic systems, the TMS coil could be navigated to target specific anatomical sites based on individual participants' structural brain images. This requires a TMS Neuronavigation system which creates a virtual link between MR images and real anatomy, and allows three-dimensional (3D) orientation by interactive visual navigation (Boroojerdi et al., 1999; Ettinger et al., 1998; Gugino et al., 2001; Herwig et al., 2002; Krings et al., 1997; T. Paus, 1999). However, although this approach accounts for inter-individual differences in anatomical brain structures, it does not account for individual differences in structure-function relationships. Stereotaxic neuronavigation can also be based on fMRI coordinates reported in the literature ["probabilistic approach"; (Paus et al., 1997)]. Hence, a third approach of positioning the TMS coil is to use frameless stereotaxy in order to navigate the TMS coil to specific standardised brain coordinates, e.g., Talairach coordinates that have been reported in the literature to be activated during tasks similar to those to be used in a TMS experiment. The coordinates used are therefore generated either from group functional imaging data, or based on a meta analysis of several studies using different imaging techniques. This approach considers both structural and functional imaging data relevant to the cognitive task to be used, but is still prey to individual structure-function differences. Finally, stereotaxic neuronavigation may be based on a 
participant's individual functional MRI data for the respective cognitive function. Based on these individual imaging results, TMS subsequently probes whether the identified task-correlated activities in these areas are necessary for successful task performance (Andoh et al., 2006; Sack, Kohler, Linden, Goebel, \& Muckli, 2006; Thiel et al., 2005). This fourth approach thus accounts for inter-individual differences both in brain anatomy and in the functional architecture of the brain.

The question to be answered is how much pre TMS anatomy is sufficient to make inferences based on TMS. This gives rise to the question of how much effort is required to optimise the accuracy of TMS coil positioning given the limits of spatial resolution of TMS. Sparing et al. (2008) have addressed this question by evaluating the accuracy and efficiency of different localisation strategies for TMS-based primary motor cortex mappings (Sparing, Buelte, Meister, Pauscaron, \& Fink, 2008) and found the highest precision with fMRI-guided stimulation, which was accurate within the range of millimeters. These findings thus indicate that even in case of simple TMS motor mapping measurements, the strength and accuracy of the induced effects are systematically modulated by the way the TMS coil is positioned above the target site. The importance of coil positioning becomes more problematic when applying TMS to silent areas in studies of cognitive functions. A recent study by Feredoes and colleagues (2007) illustrated this point by using fMRI to localise TMS sites to disrupt short-term retention of verbal information. The authors demonstrated that fMRI-guided stereotaxic neuronavigation revealed significant effects only when based on the participant's individual fMRI data, whereas the determination of the TMS target site based on spatially normalised group-average fMRI data indicated a "wrong" brain region (Feredoes, Tononi, \& Postle, 2007). Feredoes and colleagues (2007) based their conclusion on a dichotomised all-or-nothing principle, i.e. fMRI-guided neuronavigation based on single-subject analyses reveals TMS effects, while fMRI-guided neuronavigation based on group-analyses does not. However, it may be much more realistic to expect that the difference between various coil positioning approaches is gradual rather than qualitative. In order to address these issues, a systematic and quantitative assessment of the differential effect of different TMS coil positioning approaches on the induced behavioural changes during cognitive studies is needed.

In a recent cognitive TMS study (Cohen Kadosh et al., 2007), we demonstrated that a TMS-induced disruption of the right, but not left, intraparietal sulcus (IPS) significantly impairs automatic magnitude processing as measured by a reduced facilitatory component of the size congruity effect (SCE). During this study, we investigated a small number of participants $(n=5)$ with maximum precision and a high number of trials, emphasising exact individual TMS coil positioning using fMRI-guided Neuronavigation. We believed that the imaging-guided TMS Neuronavigation was a methodological prerequisite for optimally revealing the specific and significant behavioural effects of right parietal TMS on magnitude processing. However, without a direct comparison between fMRI-guided TMS Neuronavigation with other ways of targeting TMS stimulation sites, the importance of our methodological approach on the effect strength and sample size was speculative. It thus also remained unclear to what extent the revealed TMS-induced automatic number 
processing impairments during right parietal TMS may depend on how we determined target stimulation site. In the current study, we investigated whether the effects of right parietal TMS on size congruity revealed in our previous study, can be obtained when using other approaches to determine the target stimulation site. We repeated our original study testing the same number of participants $(n=5)$ under four different experimental conditions, comparing the four methods of determining the target stimulation site. We hypothesised that the strengths of the behavioural effects of TMS in cognitive studies depends on the use of an appropriate localisation strategy for positioning the TMS coil above the "silent" target stimulation site.

\section{MATERIALS AND METHODS}

\section{PARTICIPANTS}

Twenty healthy volunteers (seven males, mean age $=24$, SD $=3.9$ ) were assigned to four experimental groups of five participants. A between-subject design had to be employed since preliminary psychophysical tests showed a strong learning effect, i.e. better task performance with practice. To avoid confounds between TMS effects and task familiarity, four separate groups of randomly assigned participants were tested. Each group underwent one method of coil localisation. All participants were medically approved, had normal or corrected-to-normal vision and had no history of neurological or psychiatric disorders. All gave written informed consent after being introduced to the procedure. The study was approved by the local Medial Ethical Commission.

\section{STIMULI AND PARADIGM}

Stimuli were two digits (vertical visual angle of $0.8^{\circ}$ or $1.1^{\circ}$ ) presented centrally. The centre-to-centre distance between the two digits subtended 40 horizontally. Presentation (Neurobehavioral Systems, San Francisco, CA) stimulus-presentation software was used. Both digits varied in numerical value as well as in physical size. Participants were instructed to decide which of two stimuli in a given display was numerically larger while ignoring the physical size difference between both numbers. The numerical comparisons were performed with congruent, neutral or incongruent digit pairs. In the congruent condition, one digit is numerically and physically larger or smaller, (e.g., 24 ). In the incongruent condition, one digit is physically larger while the other is numerically larger, or vice versa (e.g., 24 ). In the neutral condition there is no difference in physical size (e.g., 24 ). Participants were instructed to indicate as quickly and accurately as possible which digit is numerically larger by pressing one of two keys corresponding to the side of the display. Previous studies show that participants unintentionally process the irrelevant dimension (Cohen Kadosh et al., 2007; Cohen Kadosh, Lammertyn, \& Izard, 2008; Henik, 1982; Tzelgov, 1992), resulting in decreased response times to congruent 
trials (facilitation) and increased response times to incongruent trials (interference) when compared to the neutral condition.

\section{MAGNETIC RESONANCE IMAGING}

Prior to TMS, high-resolution anatomical images were obtained for each participant using a 3-T magnetic resonance imaging scanner (Siemens Allegra MR Tomograph (Siemens AG, Erlangen, Germany)). The T1-weighted data set was acquired with the help of a magnetisation-prepared rapid acquisition gradient echo (MPRAGE) sequence or a T1-weighted structural scan with an isotropic resolution of $1 \mathrm{~mm}$ using a modified driven equilibrium Fourier transform (MDEFT) sequence with optimised contrast for GM and WM and imaging parameters (echo time $=4 \mathrm{~ms}$, $256 \times 256 \times 192$ matrix, voxel dimensions $=1 \times 1 \times 1 \mathrm{~mm}$ ).

\section{CORTICAL-SURFACE RECONSTRUCTION}

MR data were analysed using the BrainVoyager QX 1.8 software package (BrainInnovation, Maastricht, The Netherlands). The high-resolution anatomical recordings were used for surface reconstruction of the right hemisphere of each participant. The surface reconstruction was performed in order to recover the exact spatial structure of the cortical sheet and to improve the visualisation of the anatomical gyrification. The white-grey-matter boundary was segmented with a region growing method preceded by inhomogeneity correction of signal intensity across space. The borders of the two resulting segmented subvolumes were tessellated to produce a surface reconstruction of the right hemisphere. Subsequently, inflated cortical surfaces were created.

\section{FMRI MEASUREMENTS AND ANALYSIS}

In five participants, the MR measurements also included the acquisition of functional images while participants performed the size congruity paradigm described above. The main purpose of these fMRI measurements was to localise the exact brain activity changes underlying the described SCE individually in each participant. Functional images were acquired using a gradient-echo echo-planar imaging sequence (16 axial slices; repetition time/echo time $=2500 / 30 \mathrm{~ms}$; flip angle $=90^{\circ}$, field of view $=192 \times 192 \mathrm{~mm}$, voxel size $=3 \times 3 \times 5 \mathrm{~mm}$ ). Each functional run contained 48 trials ( 24 trials $\times 2$ congruencies [incongruent/congruent]). Stimulus presentation was synchronised with the fMRI sequence at the beginning of each trial. The first two volumes of each run were discarded to allow for T1 equilibration. The remaining functional data sets were coregistered to Talairach-transformed anatomical data (Talairach \& Tournoux, 1988) and 3D motion-corrected for each participant. The 3D functional data set was re-sampled to a voxel size of $3 \times 3 \times 3$ $\mathrm{mm}$. Further preprocessing included spatial smoothing with a Gaussian kernel (fullwidth at half-maximum $[\mathrm{FWHM}]=8 \mathrm{~mm}$ for the group analysis and $\mathrm{FWHM}=3 \mathrm{~mm}$ for the individual analysis), linear trend removal, temporal high-pass filtering (high pass: $0.00647 \mathrm{~Hz}$ ), and autocorrelation removal. The predictor time courses (boxcar functions) were convolved with a gamma distribution to account for the shape 
and delay of the hemodynamic response (Boynton, Engel, Glover, \& Heeger, 1996). Each trial began with an asterisk fixation point, presented for $500 \mathrm{~ms}$ at the centre of the screen. Five hundred ms after the fixation point disappeared, a pair of digits appeared for $1 \mathrm{~s}$ (Figure 1). The inter trial interval was $6000 \mathrm{~ms}$, and the inter block interval was at least $15 \mathrm{~s}$. Stimuli were projected (Sanyo PLC-XT16) onto a frosted screen at the rear end of the scanner bore, and viewed through a mirror mounted onto the head coil. Participants' responses were registered by a hand held fibre-optic response system (LUMItouch fMRI Optical Response keypad, Photon Control, Burnaby, Canada, www.photonixco.com). At the group level, a fixed-effect analysis was employed, with a $2 \times 2$ factorial repeated-measures design. Group effects are shown if the $p$ value yielded $p<0.05$ (Bonferroni corrected for multiple comparisons). For the individual data, the effects are shown if the $p$ value yielded $p$ $<0.001$ (uncorrected).

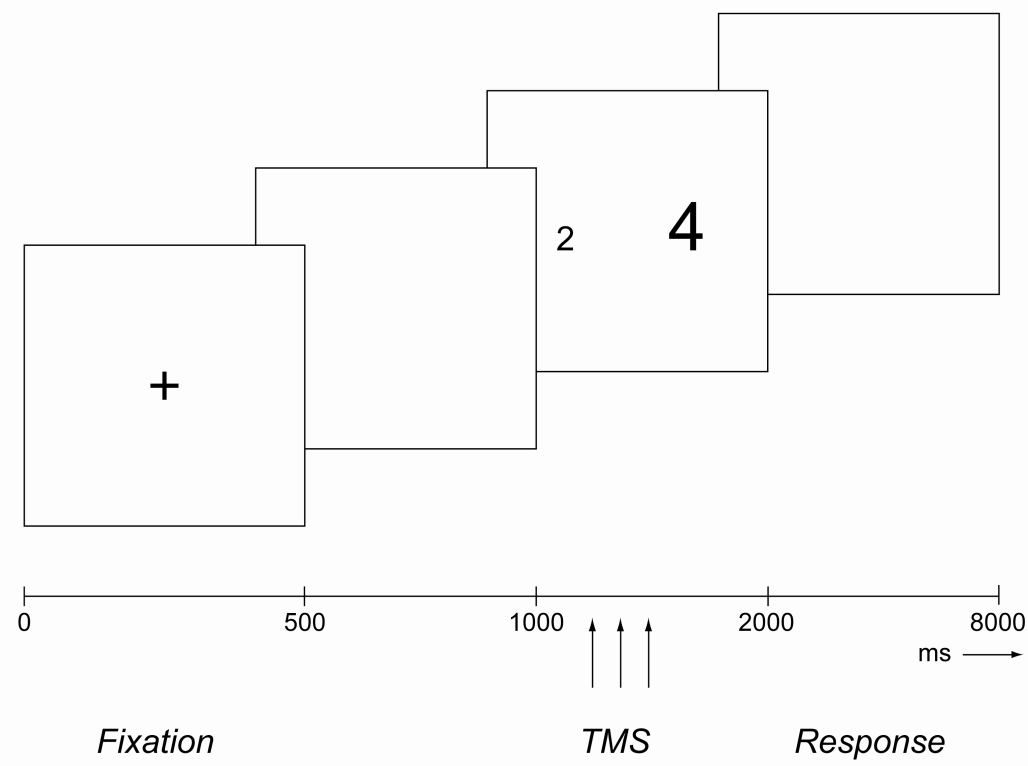

Figure 1. Size Congruity paradigm.

Each pair of stimuli was preceded by a fixation point and a blank screen (500 ms each) and remained visible for $1 \mathrm{~s}$. After $6 \mathrm{~s}$ (inter-trial interval) a new trial began with the presentation of a fixation point. The participants had to decide which stimulus was numerically larger while ignoring the physical size of the stimulus. The correct response was indicated by a button press.

\section{CO-REGISTRATION OF STEREOTAXIC AND MRI DATA}

For TMS recording and positioning we used the BrainVoyager TMS Neuronavigation system (BrainInnovation, Maastricht, The Netherlands). This system consists of 
several miniature ultrasound transmitters attached to the participant's head, and to the TMS coil. These ultrasound markers continuously transmit pulses to a receiving sensor device. Local spatial coordinate systems are created by linking the relative raw spatial position of the ultrasound senders to a set of fixed additional landmarks on the participant's head. The specification of these fixed landmarks is achieved via a digitising pen that also hosts two transmitting ultrasound markers in order to measure its relative position in 3-D space. The three anatomical landmarks used to define the local coordinate system were the nasion and the two incisurae intertragicae. The neuronavigation system thus provides topographic information of the head-based transmitters relative to a participant-based coordinate frame. After having defined the local spatial coordinate system for the participant's head and the TMS coil in real 3-D space, these coordinate systems have to be co-registered with the coordinate system of the MR space. For TMS-MRI co-registration, the same landmarks digitised on the participant's head are specified on the head reconstruction of the anatomical data from the MR sequence. After the landmarks specified on the real head have been co-registered with those on the reconstructed head, events occurring around the head of the participant in real space are registered online, and visualised and recorded in real-time at correct positions relative to the participant's anatomical reconstruction of the brain. The TMS coil can now be neuronavigated to a specific anatomical brain area or functional region of interest in every individual participant (Figure 2).

\section{LOCALISATION OF TMS TARGET REGION}

In accordance with previous findings using the identical size congruity paradigm (Cohen Kadosh et al., 2007; Cohen Kadosh, Lammertyn, \& Izard, 2008; Kaufmann et al., 2005; Pinel, Piazza, Le Bihan, \& Dehaene, 2004), we aimed to apply TMS to the right IPS in order to interfere with automatic magnitude processing. In our previous study we demonstrated that a TMS-induced disruption of right, but not left, IPS activity significantly impairs the SCE as measured by a significant decrease in the response speed facilitation during congruent stimuli. In the current study, however, we wanted to systematically investigate whether this previously revealed TMS-induced behavioural effect following fMRI-guided TMS Neuronavigation over right IPS could also be revealed with other methods of determining the right parietal target stimulation site. Therefore, we repeated our original study testing the same number of participants $(\mathrm{N}=5)$ under four different experimental conditions, systematically comparing the four different most commonly used approaches of determining the parietal target stimulation site in TMS studies. We intentionally decided to test only five participants in each of the approaches in order to investigate whether all approaches were able to reveal significant results when testing such a small number of participants, as individual fMRI based stereotaxic neuronavigation had accomplished. 


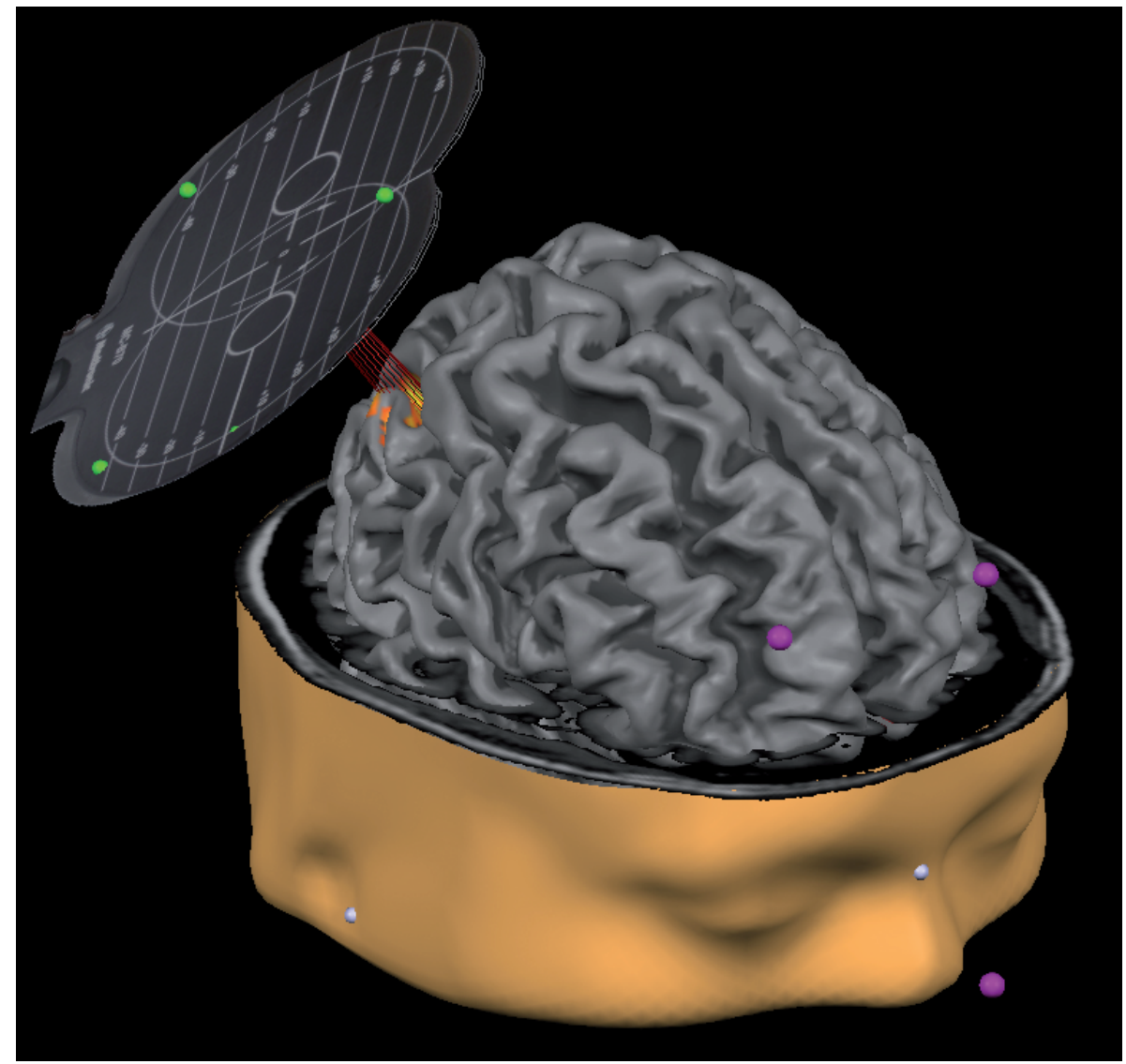

Figure 2. TMS Neuronavigation based on individual fMRI data.

An example of functional data superimposed on the anatomical reconstruction of the brain and the coregistered TMS coil.

\section{FOUR DIFFERENT APPROACHES OF DETERMINING THE PARIETAL TMS STIMULATION SITE}

\section{A) FMRI-guided TMS neuronavigation}

For one group the right parietal TMS target site was determined as the individual location within the intraparietal sulcus (IPS) exhibiting the strongest BOLD-signal contrast for the SCE. In order to achieve this precision, the functional data of each individual participant was superimposed on the individual anatomical reconstruction of the brain. In every participant, the TMS coil was then neuronavigated using frameless stereotaxy in order to stimulate the specific functional activation area in right IPS underlying the SCE. In contrast to the fMRI group analysis, TMS neuronavigation was based on individual data in AC-PC space (the cerebrum was rotated into the anterior commissure-posterior commissure plane). This was done in order to avoid any additional transformations that could distort the correspondence between MRI and stereotaxic points. 


\section{B) MRI-guided TMS neuronavigation}

The parietal TMS target site for the second group was determined based on individual MRI data. For each participant an anatomical reconstruction of the right hemisphere was created. Prior to the experiment, the target site was individually determined as the anterior part of the intraparietal sulcus (IPS). The TMS neuronavigation system was employed to navigate to this anatomically defined target region in each participant.

\section{C) TMS neuronavigation based on group Talairach coordinates}

For the third group, the parietal TMS target site was determined as the Talairach coordinates of the location in the fMRI group analysis which exhibited the strongest SCE within right IPS (Cohen Kadosh et al., 2007; Talairach coordinates $[x, y, z]=$ $[22,-68,39])$. These normalised stereotaxic coordinates were marked as target sites on the normalised brain of each participant. This location was then back transformed to the individual participant's brain coordinates in native space by reversing the native-to-Talairach transformation procedure. Finally, the TMS neuronavigation system was used to navigate to this group average target site on the basis of each individual brain anatomy in AC-PC space.

\section{D) TMS based on the 10-20 EEG system (anatomical landmark approach)}

For the fourth group, the International 10-20 system for EEG electrode placement (Jasper, 1958) was employed for determination of the parietal TMS target site. A lycra swimming cap was placed on the participant's head in order to mark the location of P4, which has been shown to correspond to right IPS (Herwig, Satrapi, \& Schönfeldt-Lecuona, 2003). Although placement of the TMS coil required no TMS neuronavigation, it was still used in order to determine in every single participant which exact anatomical brain structure was actually underlying P4 and thus stimulated during this approach.

\section{EXPERIMENTER BIAS}

The stereotaxic system employed in this study allows real-time and online TMS neuronavigation to any predefined TMS target site. Importantly, the accuracy of coil positioning is monitored and recorded throughout the entire experiment. Therefore it is certain that a target site is stimulated equally precise in all four conditions, excluding the possibility that differential results between conditions are due to differences in stimulation accuracy, e.g., due to random fluctuations or an experimenter bias.

\section{TMS PROTOCOL}

Biphasic TMS pulses were applied with a figure-of-eight coil (MCB70; inner and outer radii of the two coil loops $=1.2 \mathrm{~cm}$ and $5.4 \mathrm{~cm}$, respectively) and a Medtronic MagPro X100 stimulator (Medtronic Functional Diagnostics A/S, Skovlunde, Denmark; maximum stimulator output, $2 \mathrm{~T}$ ). The coil was fixed on a tripod and placed tangentially on the skull with a custom-made coil holder. The coil position was monitored online using the BrainVoyager TMS Neuronavigation system. Triple-pulse 
TMS at $10 \mathrm{~Hz}$ was applied at $60 \%$ of maximum stimulator output. The pulses were given at $220 \mathrm{~ms}, 320 \mathrm{~ms}$, and $420 \mathrm{~ms}$ after stimulus presentation. The timing of the pulses was chosen based on a recent ERP study that found increased amplitudes in the congruency conditions at the parietal electrode around $220 \mathrm{~ms}$ after stimulus presentation. These amplitudes peaked around 350-400 ms after stimulus presentation. For the sham condition, TMS pulses were applied using a placebo figure-of eight TMS coil (MC-P-B70 Placebo). The positioning and navigation of the placebo coil, as well as the applied stimulation parameters, were the same as those in the real TMS condition. Each participant underwent a TMS session, in which the order of real and sham stimulation was counterbalanced between the participants. Congruent, neutral, and incongruent conditions were randomly sampled, and there was an equal sampling for each condition. A total of 144 trials were presented to each participant (24 trials $\times 2$ sessions [TMS, sham] $\times 3$ congruencies [incongruent, neutral, and congruent]). Responses had to be made equally often with the left and right hand.

\section{STATISTICAL ANALYSIS OF BEHAVIOURAL DATA}

Mean RTs for every participant in each condition were calculated for correct trials only. RTs that were 2.5 standard deviations from the mean of each condition for each individual were excluded (less than $2 \%$ ). An outlier analysis was performed per approach (group mean +/- 2.5 SD), ensuring that the group means were not distorted by extreme values of a single participant. RTs were analysed for all four approaches together, and subsequently for the conditions separately using a $2 \times 3$ factorial repeated-measures ANOVA (Stimulation [TMS/sham] x Congruency [incongruent/neutral/congruent]). In order to asses the facilitatory effect more directly, a $2 \times 2$ factorial repeated-measures ANOVA was performed (Stimulation [TMS/sham] $x$ Congruency [neutral/congruent]). Additionally, one-sample t-tests were performed on the difference between sham and TMS in SCE (calculated as RT[incongruent] - RT[congruent]) for each of the approaches separately, which allowed an estimation of the effect-size obtained by each of the four approaches.

\section{RESULTS}

\section{BEHAVIOURAL RESULTS WITHOUT TMS: THE SIZE CONGRUITY EFFECT}

The task performance during baseline elicited a strong and significant SCE as measured by a significant difference between congruent and incongruent trials both in terms of reaction times ([RT(incongruent) $-\mathrm{RT}$ (congruent) $=104 \mathrm{~ms}, \mathrm{SD}=$ 44.3]; $\mathrm{t}=10.530, \mathrm{p}$ (one-tailed) <.001), and error rates ([error rate (incongruent) - error rate (congruent) $=.200, \mathrm{SD}=.142] ; \mathrm{t}=6.320, \mathrm{p}$ (one-tailed) < $.001)$. This significant SCE was comprised of a significant facilitatory component $([\mathrm{RT}$ (neutral) $-\mathrm{RT}$ (congruent) $=36 \mathrm{~ms}, \mathrm{SD}=20.9] ; \mathrm{t}=7.766, \mathrm{p}$ (one-tailed) < .001 ) as well as a significant interference component ([RT(incongruent) $\mathrm{RT}($ neutral) $=68 \mathrm{~ms}, \mathrm{SD}=41.4] ; \mathrm{t}=7.346, \mathrm{p}$ (one-tailed) $<.001$ ). Whereas the facilitatory effect reveals that participants were significantly faster in their decision 
when the task-irrelevant dimension of the stimuli (physical size) was in accordance with the task-relevant dimension (numerical size), the interference effect demonstrates a significant decrease in performance when the task-irrelevant dimension was in conflict with the task-relevant dimension.

\section{FMRI DATA}

FMRI was employed in order to localise the exact neural correlates underlying this behavioural SCE. A contrast analysis between congruent and incongruent trials during fMRI revealed higher activation in bilateral premotor regions (the putative frontal eye fields and the presupplementary motor area) and the right frontal operculum. Several of these areas have been reported in studies that utilised conflict situations (Aron, Robbins, \& Poldrack, 2004; Critchley, Tang, Glaser, Butterworth, \& Dolan, 2005; Picard \& Strick, 2001; Rushworth, Walton, Kennerley, \& Bannerman, 2004). Moreover, in line with previous studies (Kaufmann et al., 2005; Pinel, Piazza, Le Bihan, \& Dehaene, 2004) the SCE activated intraparietal sulcus (IPS) bilaterally (Figure 3 ). Our previous study ascertained that within this bilateral parietal activation network only the right IPS activity is functionally necessary for the facilitatory component of the SCE as revealed by fMRI-guided TMS Neuronavigation (Cohen Kadosh et al., 2007). Based on this result we targeted only the right IPS in the subsequent TMS studies, aiming to replicate our previous findings of a TMSinduced reduction of the facilitatory component of the SCE to compare the four different approaches of targeting the exact stimulation site within right IPS: i) fMRIguided TMS Neuronavigation, ii) MRI-guided TMS Neuronavigation, iii) TMS Neuronavigation based on group Talairach Coordinates, and iv) TMS over P4.

\section{EFFECTS OF FMRI-GUIDED TMS NEURONAVIGATION}

In order to accurately stimulate the functionally defined region of interest along the right IPS in each participant, the IPS SCE of the fMRI data was analysed separately for each individual participant. The results revealed considerable interindividual differences in the IPS activation both in extent and in spatial layout and, therefore, in the stimulated coordinates (Figure 3; Table 2).

\section{Sham TMS}

When stimulating this functionally defined right IPS activation using a TMS placebo coil (sham condition), we again revealed a clear and significant SCE $[t=4.073, p$ (one-tailed) <.01], calculated as [RT(incongruent) - RT(congruent)]. More concretely, RTs during congruent trials $(466 \mathrm{~ms}, \mathrm{SD}=39.4)$ were significantly faster as compared to incongruent trials ( $579 \mathrm{~ms}, \mathrm{SD}=88.7$ ). This SCE was comprised of both, a significant facilitatory component (44 ms, SD $=4.6 ; t=21.465, p$ (onetailed $)<.001)$, as well as a significant interference effect $(69 \mathrm{~ms}, \mathrm{SD}=62.9 ; \mathrm{t}=$ 2.467, p (one-tailed) <.05). 


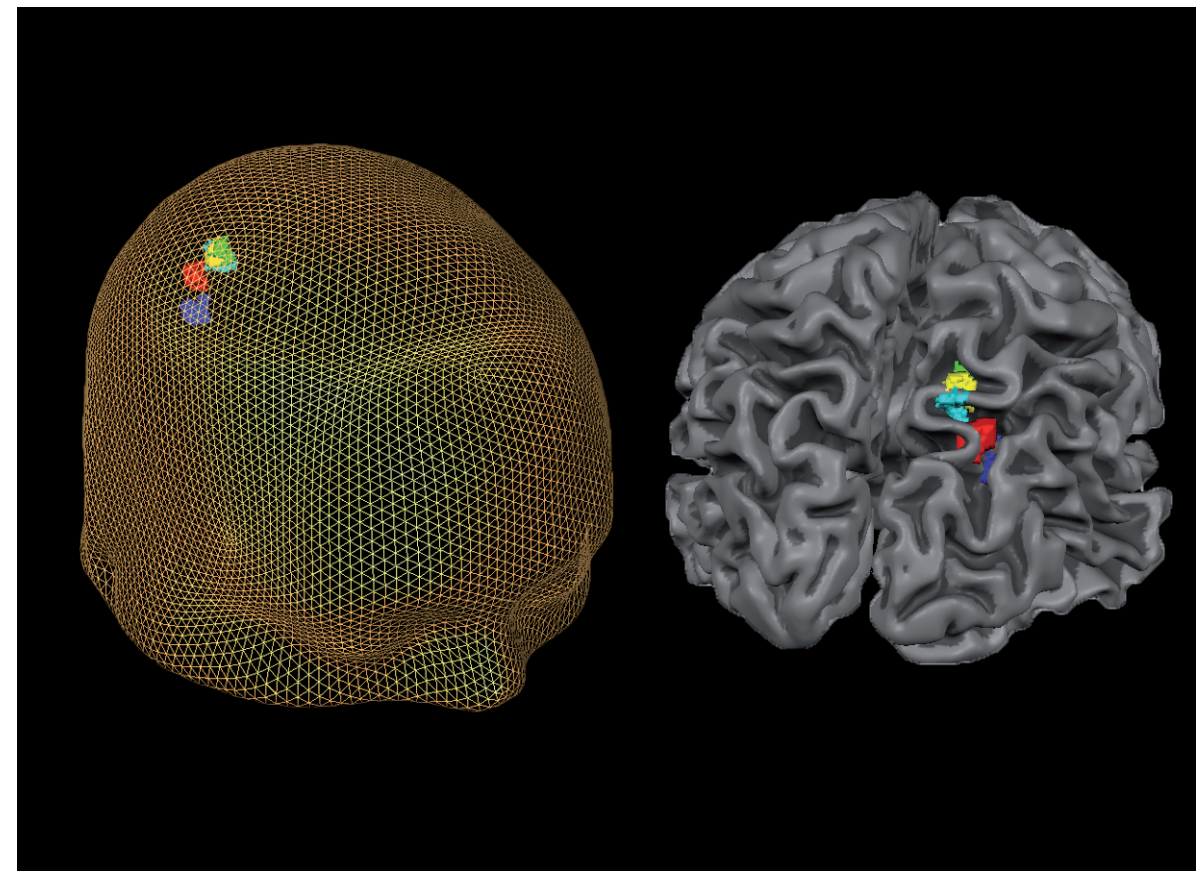

Figure 3. Individual differences in brain activity.

The different colours represent the neural correlates underlying the behavioural SCE for the individual participants.

\section{Right IPS}

A direct statistical contrast between fMRI-guided real versus sham TMS over right IPS revealed that during real TMS the overall SCE (RT(incongruent) $\mathrm{RT}$ (congruent) $=80 \mathrm{~ms}, \mathrm{SD}=48.0$ ) was significantly reduced $(\mathrm{t}=2.517, \mathrm{p}$ (onetailed) < 0.05; see Figure 4) as compared to sham TMS (RT(incongruent) $\mathrm{RT}$ (congruent) $=113 \mathrm{~ms}, \mathrm{SD}=62.4)$. Importantly, a two-way repeated measures ANOVA with TMS (sham versus real TMS) and congruency (neutral or congruent) as the two within subject factors revealed a significant interaction between TMS and congruency $[F(1,4)=7.677, p<.05]$.

\section{EFFECTS OF MRI-GUIDED TMS NEURONAVIGATION}

\section{Sham TMS}

During sham TMS we again revealed a clear and significant SCE $[t=39.617, p$ (one-tailed) <.001]. More concretely, RTs during congruent trials (439 ms, SD = 37.0) were significantly faster as compared to incongruent trials ( $564 \mathrm{~ms}, \mathrm{SD}=$ 37.2). This SCE was comprised of both, a significant facilitatory component ( $43 \mathrm{ms,}$ $\mathrm{SD}=24.4 ; \mathrm{t}=3.899, \mathrm{p}$ (one-tailed) $<.05$ ), as well as a significant interference effect $(82 \mathrm{~ms}, \mathrm{SD}=20.9 ; \mathrm{t}=8.743$, $\mathrm{p}$ (one-tailed) $<.01)$. 


\section{Right IPS}

When stimulating this anatomically defined right IPS using real TMS we also revealed a significant SCE $[t=8.143, \mathrm{p}$ (one-tailed) $<.001]$. Although the SCE was descriptively reduced during real TMS (RT(incongruent) $-\mathrm{RT}$ (congruent) $=102 \mathrm{~ms}$, $\mathrm{SD}=27.9$ ) as compared to sham TMS (RT(incongruent) $-\mathrm{RT}$ (congruent) $=124$ $\mathrm{ms}, \mathrm{SD}=7.0$ ), a direct post hoc contrast between both TMS conditions did not find this difference to be significant $(t=1.834, p$ (one-tailed) $=.071$; see Figure 4 ). Accordingly, a two-way repeated measures ANOVA with TMS (sham versus real TMS) and congruency (neutral or congruent) as the two within subject factors also failed to reveal a significant interaction $[F(1,4)=2.504, p=.189]$.

\section{EFFECTS OF TMS NEURONAVIGATION BASED ON TALAIRACH COORDINATES}

\section{Sham TMS}

During sham TMS we revealed a clear and significant SCE $[\mathrm{t}=4.183, \mathrm{p}$ (one-tailed) $<.01]$. More concretely, RTs during congruent trials $(407 \mathrm{~ms}, \mathrm{SD}=33.1$ ) were significantly faster as compared to incongruent trials (484 ms, SD $=71.3$ ). This SCE was comprised of both a significant facilitatory component ( $25 \mathrm{~ms}, \mathrm{SD}=24.3$; $\mathrm{t}=2.284$, $\mathrm{p}$ (one-tailed) <.05), as well as a significant interference effect (53 ms, $\mathrm{SD}=36.6 ; \mathrm{t}=3.235, \mathrm{p}$ (one-tailed) $<.05)$.

\section{Right IPS}

When stimulating right IPS based on Talairach group coordinates using real TMS we also revealed a significant SCE $[t=5.548, \mathrm{p}$ (one-tailed) $<.01]$. Although the SCE was descriptively reduced during real TMS (RT(incongruent) $-\mathrm{RT}$ (congruent) $=59$ $m s, S D=23.7$ ) as compared to sham TMS (RT(incongruent) $-\mathrm{RT}$ (congruent) $=78$ $\mathrm{ms}, \mathrm{SD}=41.6)$, a direct post hoc contrast between both TMS conditions did not find this difference to be significant $(t=1.487, p$ (one-tailed) $=.106$; see Figure 4). Accordingly, a two-way repeated measures ANOVA with TMS (sham versus real TMS) and congruency (neutral or congruent) as the two within subject factors also failed to reveal a significant interaction $[F(1,4)=0.10, p=.54]$.

\section{EFFECTS OF TMS OVER P4}

\section{Sham TMS}

During sham TMS we revealed a clear and significant SCE [ $\mathrm{t}=4.891, \mathrm{p}$ (one-tailed) $<.01]$. More concretely, RTs during congruent trials (426 ms, SD $=56.0$ ) were significantly faster as compared to incongruent trials (528 ms, SD $=90.5)$. This SCE was comprised of both a significant facilitatory component ( $34 \mathrm{~ms}, \mathrm{SD}=23.8$; $\mathrm{t}=3.159, \mathrm{p}$ (one-tailed) <.05), as well as a significant interference effect (68 ms, $\mathrm{SD}=43.4 ; \mathrm{t}=3.494, \mathrm{p}$ (one-tailed) $<.05)$.

\section{Right IPS}

When stimulating P4 based on the 10-20 EEG system using real TMS we also revealed a significant SCE $[t=3.338, \mathrm{p}$ (one-tailed) $<.05]$. Although the SCE was descriptively reduced during real TMS (RT(incongruent) $-\mathrm{RT}$ (congruent) $=86 \mathrm{~ms}$, 
$\mathrm{SD}=57.5)$ as compared to sham TMS (RT(incongruent) $-\mathrm{RT}$ (congruent) $=101$ ms, SD = 46.4), a direct post hoc contrast between both TMS conditions did not find this difference to be significant $(t=.749, p$ (one-tailed) $=.247$; see Figure 4 ). Accordingly, a multivariate two-way repeated measures ANOVA with TMS (sham versus real TMS) and congruency (neutral or congruent) as the two within subject factors also failed to reveal a significant interaction $[F(1,4)=.83, p=.41]$.

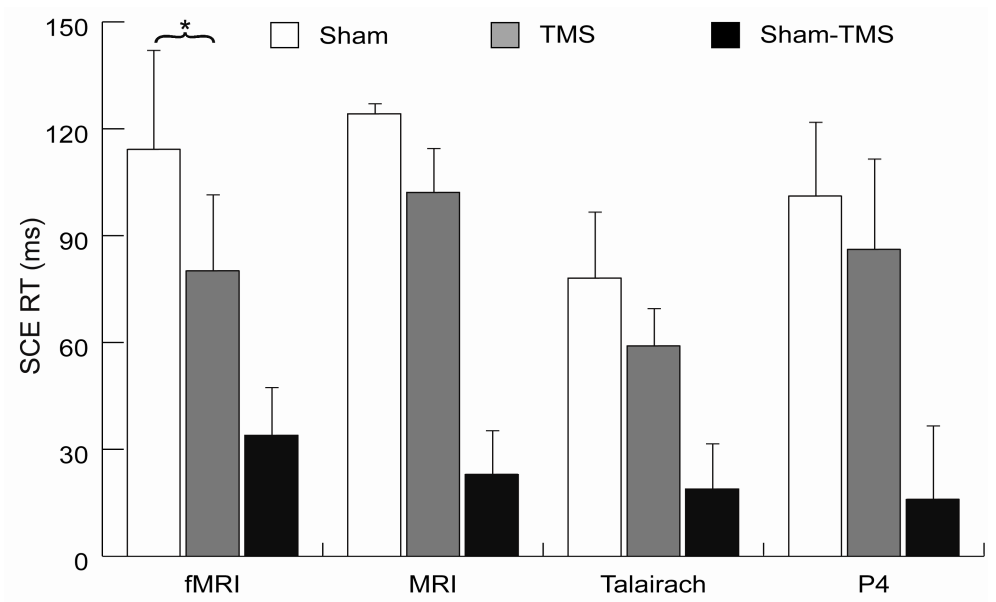

Figure 4. Size congruity effect as a function of the four approaches for TMS target site determination.

White, grey, and black bars reflect the SCE for sham, TMS, and the difference between sham and TMS respectively. Error bars depict one standard error of the mean (SEM). The asterisk indicates a significant difference between SCE(sham) and SCE(TMS) at $a=0.05$.

\section{DIRECTLY COMPARING THE EFFECT SIZES OF THE FOUR APPROACHES}

Our results indicate that when the right IPS is stimulated in the location indicated by the individual fMRI results, the SCE decreases significantly. Importantly, this effect was only found to be significant with $n=5$ when using fMRI-guided TMS neuronavigation to approach the TMS target site, but could not be replicated using a different approach for TMS target site determination testing the same number of participants. Although all applied TMS approaches showed the same trend as the fMRI-guided TMS neuronavigation, the effect size was not large enough to reach statistical significance. In the next stage of analysis we computed the power of these experiments to determine how many participants would be needed for each of the three reductions in SCE to become significant.

In contrast to just comparing significance differences between groups, the effect size is a standardised measure of the real effect which takes into account not only the observed difference in RTs, but also the variability, the alpha error, the beta error, the test power, and the sample size, making it a valid measure of the standardised strength of any observed effect (Cohen, 1988). Importantly, effect 
sizes enable a parametric differentiation and ordering within and between several significant or non-significant results.

When directly comparing the four approaches for TMS target site determination, a clear difference occurs in the strength of the effect size of TMS (Table 1). As discussed in the previous section, for all four approaches the SCE was reduced in the TMS condition as compared to the sham condition. However, when calculating the effect size of this difference, the effect in the fMRI-guided TMS neuronavigation was largest (Cohen's $d=1.13$ ), followed by the effect size for the MRI-guided TMS neuronavigation (Cohen's $d=0.82$ ), followed by the effect size for the TMS neuronavigation based on group Talairach coordinates (Cohen's $d=0.67$ ), and finally smallest for the TMS over P4 approach (Cohen's $d=0.34$ ). The effect sizes for the interaction between TMS and congruency as shown by the two-way repeated measures analysis of variance (ANOVA) with TMS (sham or TMS) and congruency (incongruent, neutral, or congruent) as within-subject factors showed the same order in effect sizes between the four approaches. Again, fMRI-guided TMS neuronavigation generated the largest effect size $\left(f^{2}=1.23\right)$, followed by MRI-guided TMS neuronavigation ( $f^{2}=0.91$ ), neuronavigation based on Talairach coordinates ( $f^{2}=$ $0.34)$, and TMS stimulation over P4 ( $\left.\mathrm{f}^{2}=0.13\right)$.

Table 1. Effect sizes of paired-samples t-tests on the difference in SCE between sham and TMS.

Effect size (Cohen's d)

\begin{tabular}{ll}
\hline fMRI & 1.13 \\
MRI & 0.82 \\
Talairach & 0.67 \\
Anatomical & 0.34 \\
\hline
\end{tabular}

\section{OPTIMAL SAMPLE SIZE}

The optimal sample size of an experiment can theoretically be defined as the exact number of participants necessary to reveal an a priori determined empirical effect of a certain size, or any greater size, as statistically significant. This implies that any smaller empirical effect, even though potentially statistically significant at a greater sample size, will not reach statistical significance under these circumstances. Statistical power analysis enables the determination of an optimal sample size by a priori specifying the $\mathrm{H} 1$ parameter and thus controlling not only for the alpha (a)- but also the beta ( $\beta$ )-error-probability of the statistical analysis. Speaking in statistical terms, the exact pre-determination of a $\mathrm{HO}$ and a $\mathrm{H} 1$ parameter enables one to calculate the probability of an empirical effect (and all higher effects) in case of a valid HO (a-error-probability), as well as to calculate the probability of an empirical effect (and all smaller effects) in case of a valid H1 ( $\beta$-errorprobability). The significance level $(a)$, the test power $(1-\beta)$, the experimental effect $\left(n^{2}\right)$ and the sample size $(n)$ are interdependent, and thus, after determining 
any three of these parameters, it is possible to calculate the fourth. In the current study, the significance level for both, $a$ - and $\beta$-error-probability, are conventionally defined. We moreover calculated the exact standardised effect size for each of the four approaches. Hence, three of the four parameters are set, enabling the calculation of the exact number of participants required for yielding each of the described effect sizes as being significant. This power analysis revealed that while in the fMRIguided neuronavigation experiment five participants were sufficient for finding a significant difference between sham and TMS, this number of necessary participants increased to $n=9$ when employing MRI-guided neuronavigation, to $n=13$ in case of TMS based on group Talairach coordinates, and finally to $n=47$ when applying TMS over P4 (Figure 5). Hence, although all four approaches reveal the same direction of effect, they differ in the number of participants needed to make any claims regarding statistical significance.

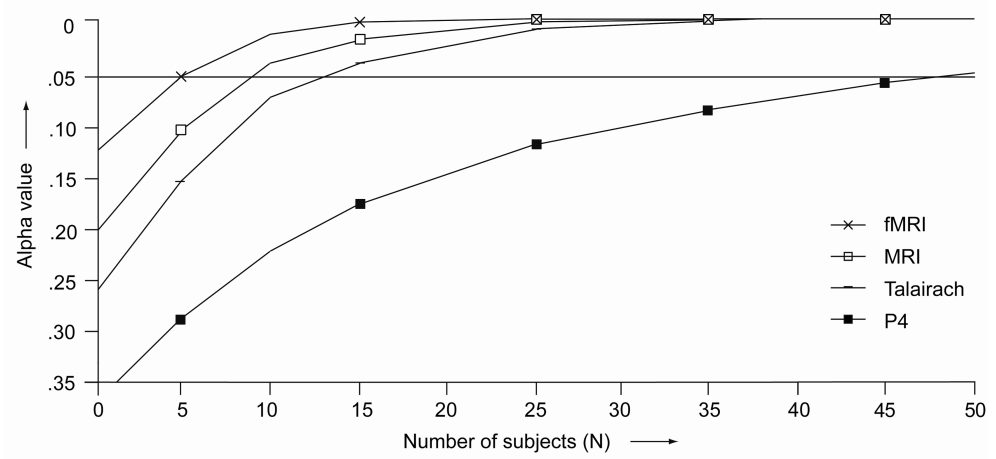

Figure 5. Relation between sample size and alpha (a) value for each of the four approaches. The different markers indicate the four approaches for determination of TMS stimulation site. The horizontal line indicates the alpha (a) value of 0.05 .

\section{QUANTIFICATION OF INDIVIDUAL VARIABILITY IN TMS TARGET SITE}

While all four approaches of determining the optimal TMS target stimulation site attempted to denote the same area within right IPS as target site, their systematic difference in TMS-induced behavioural effect sizes suggest that this has not been the case. In order to quantify the difference in the actual stimulation site between approaches that might give rise to these systematic effect size differences, the coordinates of the exact actual stimulation site in each individual participant were assessed and analysed using frameless stereotaxy (Figure 6; Table 2). 


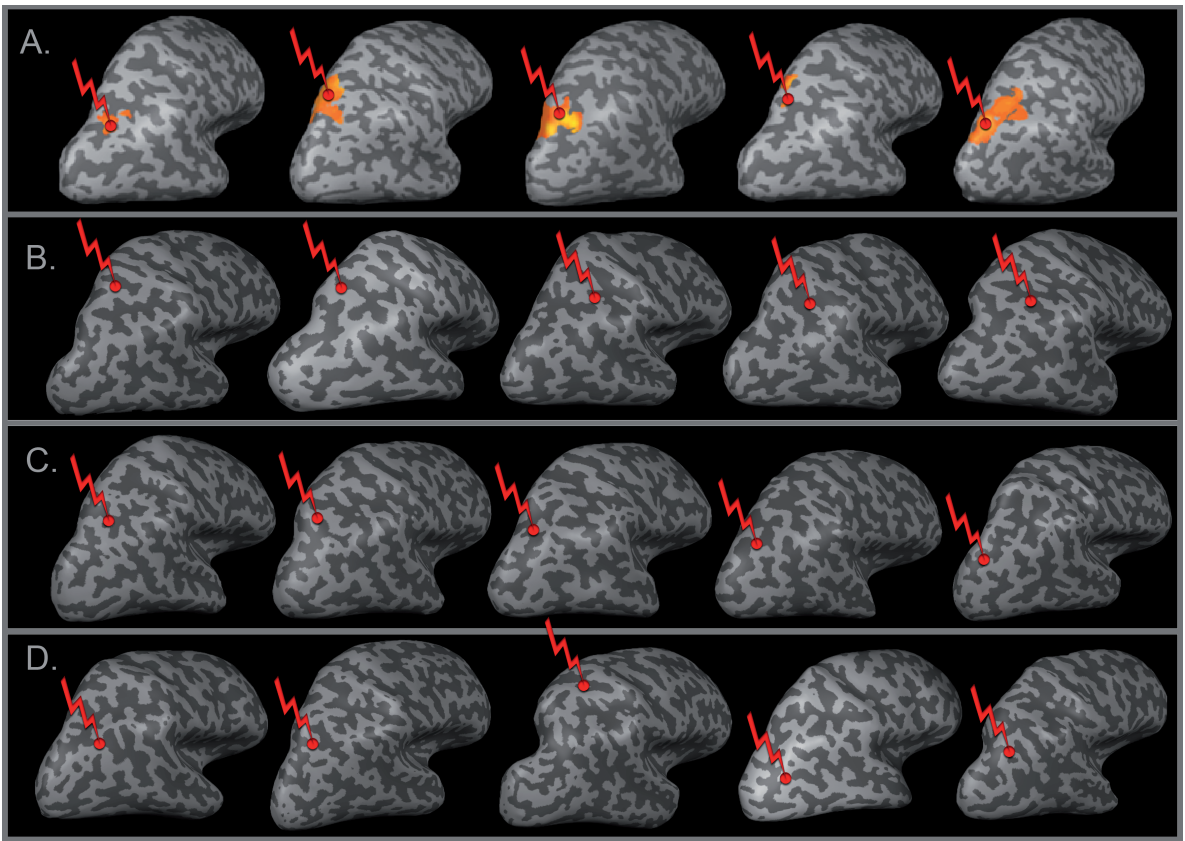

Figure 6. Segmentations of the individual participants and their TMS stimulation site.

The top row shows IPS activation as a function of the SCE in right hemisphere (A). The capital letters indicate the approaches for TMS target site determination, with (A) for fMRI-guided Neuronavigation, (B) for MRI-guided Neuronavigation, (C) for Neuronavigation based on Talairach coordinates, and (D) for TMS based on the 10-20 EEG system. The red spheres indicate the site of the maximal stimulation.

As can be seen in Table 2 (see also Figure 6), the actual TMS target site was considerably different in each participant. During the fMRI-guided TMS Neuronavigation approach, the individual fMRI results led to a large interindividual variability of the stimulated "hot spot" defined as the strongest fMRI activity underlying the SCE (SD in $x=5.6, y=4.7, z=8.6)$. The anatomically defined right IPS target site resulted in a considerable interindividual variance in corresponding Talairach coordinates (SD in $x=10.9, y=6.2, z=2.4$ ), and even larger interindividual variances were revealed in the individual Talairach coordinates underlying the P4 target TMS stimulation site (SD in $x=7.0, y=19.8, z=19.2$ ). 
Table 2. Talairach coordinates of target sites for each participant and Euclidian distance of the individual target site to site I (Talairach coordinates $[22,-68,39]$ ) and to site II (Talairach coordinates [37, $46,42])$.

\begin{tabular}{|c|c|c|c|c|c|c|}
\hline Approach & Participant & $\mathbf{x}$ & $\mathbf{y}$ & $\mathbf{z}$ & $\begin{array}{c}\text { Distance I } \\
(\mathbf{m m})\end{array}$ & $\begin{array}{c}\text { Distance II } \\
(\mathbf{m m})\end{array}$ \\
\hline \multirow[t]{5}{*}{ fMRI } & $\mathrm{P} 1$ & 29 & -67 & 28 & 13 & 26 \\
\hline & $\mathrm{P} 2$ & 16 & -70 & 47 & 13 & 32 \\
\hline & P3 & 23 & -72 & 37 & 5 & 30 \\
\hline & P4 & 18 & -60 & 47 & 12 & 24 \\
\hline & P5 & 16 & -70 & 47 & 10 & 32 \\
\hline \multirow[t]{5}{*}{ Anatomical } & P6 & 21 & -58 & 52 & 16 & 22 \\
\hline & P7 & 20 & -59 & 51 & 15 & 23 \\
\hline & P8 & 41 & -46 & 47 & 30 & 6 \\
\hline & P9 & 40 & -48 & 47 & 28 & 6 \\
\hline & P10 & 40 & -48 & 48 & 28 & 7 \\
\hline Talairach & P11-15 & 22 & -68 & 39 & 0 & 27 \\
\hline \multirow[t]{5}{*}{ P4 } & P16 & 37 & -73 & 37 & 16 & 27 \\
\hline & P17 & 32 & -82 & 28 & 17 & 39 \\
\hline & P18 & 19 & -37 & 67 & 42 & 32 \\
\hline & P19 & 33 & -86 & 15 & 32 & 48 \\
\hline & P20 & 34 & -79 & 33 & 19 & 34 \\
\hline
\end{tabular}

Notes. Talairach conventions: $\mathrm{x}$-left to right, $\mathrm{y}$-back to front, $\mathrm{z}$-bottom to top.

In order to assess and better compare the variability of the TMS target site between the four different TMS approaches, Euclidian Distances (ED) between the actual target site of stimulation and two reference target points were calculated. The first reference target point was defined as the mean Talairach coordinates of the fMRI group analysis which exhibited the strongest SCE (Site I; Talairach coordinates $[x$, $y, z]=22,-68,39)$. Although this site is likely to most closely resemble the true target area, it may bias calculations of variability. This reference target point is calculated from fMRI data acquired from participants in the first approach, which may lead to an underestimation of variability in this approach. Therefore, Euclidian Distances were additionally calculated to a reference target point based on a recent meta-analysis (Cohen Kadosh, Lammertyn, \& Izard, 2008; Site II; Talairach coordinates $[x, y, z]=37,-46,42)$. This enabled us to indicate in millimetres the exact distance from each actual TMS target stimulation site per participant and per approach to both of these reference points. For the fMRI-guided TMS approach, the average ED between each individual target TMS site and site I was $11 \mathrm{~mm}$ (SD = 3.5; variance $=12.2$ ). For the MRI-guided TMS approach, the average ED was 24 $\mathrm{mm}(\mathrm{SD}=7.2$; variance $=52.4)$, and for the P4 approach $25 \mathrm{~mm}(\mathrm{SD}=11.7$, variance $=136.7)$. Euclidian distance to site II was $29 \mathrm{~mm}(\mathrm{SD}=3.6$; variance $=$ 
13.1) for the fMRI-guided TMS approach, $13 \mathrm{~mm}(\mathrm{SD}=8.9$; variance $=79.6)$ for the MRI-guided TMS approach, and $36 \mathrm{~mm}(\mathrm{SD}=8.0$, variance $=63.4)$ for the P4 approach (Table 3 ). This again clearly demonstrated how much the exact site of stimulation can vary between participants and between approaches. The maximal difference between the reference target point (site I) and the actual TMS stimulation site was found to be as large as $42 \mathrm{~mm}$, revealed in one participant stimulated with TMS over P4 (Table 3).

Table 3. Averaged Euclidian distance of the individual target site to site I (Talairach coordinates [22, -68 , 39]) and site II (Talairach coordinates [37, -46, 42]), averaged per approach for TMS target site determination.

\begin{tabular}{lcccccc}
\hline Approach & $\begin{array}{c}\text { Distance I } \\
(\mathbf{m m})\end{array}$ & SD & variance & $\begin{array}{c}\text { Distance II } \\
(\mathbf{m m})\end{array}$ & SD & variance \\
\hline fMRI & 11 & 3.5 & 12.2 & 29 & 3.6 & 13.1 \\
Anatomical & 24 & 7.2 & 52.4 & 13 & 8.9 & 79.6 \\
Talairach & 0 & 0 & 0 & 27 & 0 & 0 \\
P4 & 25 & 11.7 & 136.7 & 36 & 8.0 & 63.4 \\
\hline
\end{tabular}

\section{DISCUSSION}

Our aim in this study was to quantify the influence of different TMS coil positioning approaches on TMS-induced behavioural effects in cognitive studies. In all four TMS positioning approaches, participants were stimulated using either real or sham TMS. In addition, a no-TMS baseline was recorded. The results and subsequent power calculations revealed the relative efficacy of the four methods compared.

Without TMS, participants showed a significant SCE as measured by a significant difference between congruent and incongruent trials for both reaction times and errors. This significant SCE was comprised of a facilitatory component, revealing that participants were faster in their decision when the task-irrelevant dimension of the stimuli (physical size) was congruent with the task-relevant dimension (numerical size), as well as a interference component, revealing a decrease in performance in case of a dimension conflict. In all four approaches sham TMS had no effect on this SCE. However, when contrasting real TMS versus sham TMS, thus calculating the net impact of right parietal TMS on the SCE, a systematic difference between the four approaches was found. For the individual fMRI-guided TMS Neuronavigation approach, a significant difference between real and sham TMS in terms of a significantly reduced SCE was revealed. Moreover, there was a significant interaction between TMS and congruency. The other three approaches of TMS coil positioning showed a reduced SCE smaller than that obtained with individual fMRIguided TMS Neuronavigation. This suggests that the four different TMS coil positioning approaches do not differ qualitatively in their TMS-induced effects but in the magnitude of their respective effect sizes and thus in the number of participants required to reveal the observed TMS-induced changes as being statistically signifi- 
cant. Accordingly, when calculating the effect size of the SCE reduction per approach, the effect size in the fMRI-guided TMS neuronavigation was largest (Cohen's $d=1.13$ ), followed by the effect size for the MRI-guided TMS neuronavigation (Cohen's $d=0.82$ ), the effect size for the TMS neuronavigation based on group Talairach coordinates (Cohen's $d=0.67$ ), and finally smallest for the TMS over P4 approach (Cohen's $d=0.34$ ). A subsequent power analysis revealed that while for fMRI-guided neuronavigation five participants were sufficient to find a significant effects, the number of necessary participants increased to $n=9$ for MRIguided neuronavigation; to $n=13$ for TMS based on group Talairach coordinates; and to $n=47$ when applying TMS over P4.

Initially, all four approaches of positioning the TMS coil attempted to denote the same area within right IPS. Yet, the differences in the TMS-induced effect sizes show this was not the case. In order to quantify the differences in the parietal target stimulation site within and between the four approaches that might underlie these effect size differences, we also assessed the brain coordinates of the actual parietal stimulation site in each participant using frameless stereotaxy. This calculation revealed that the actual TMS target site differed considerably between participants and within methods. The variability between participants and within methods was assessed by calculating the variance in Euclidian Distance to two reference sites: the mean group fMRI coordinates of the current participants sample (site I) and standardised Talairach coordinates of a respective meta study derived from the literature (site II). Although mean distances indeed differed between these two references, both sites supported the same conclusion of the variability being largest when targeting P4 and structural MRI guided anatomical sites, followed by fMRI guided sites.

In general, this inter-individual variance within each approach is neither surprising nor necessarily problematic. Large inter-subject variability has been reported along the IPS, both anatomically (Zilles, Eickhoff, \& Palomero-Gallagher, 2003) and functionally (Cohen Kadosh et al., 2007). With regard to the question of an optimal TMS coil positioning approach, this quantified inter-individual difference of parietal fMRI activity in fact demonstrates the necessity of considering this variability in order to avoid sub optimal stimulation. Indeed, neglect of this functional inter-subject variability and the resulting mistargetting of magnetic stimulation may have contributed, at least partly, to the null results of previous TMS interference studies using magnitude comparison tasks ( $T$. Gebuis, unpublished Master thesis, Maastricht University, The Netherlands), including the size congruity paradigm (Walsh, 2003). Here, we accounted for this functional variability using individual fMRI-guided TMS Neuronavigation, and by doing so, we indeed revealed the strongest behavioural effects compared to all other TMS coil positioning approaches. The second strongest effect sizes were revealed when using MRI-guided TMS Neuronavigation. Although this approach does consider the inter-individual variance in anatomical brain structure, it, unlike the fMRI-guided Neuronavigation approach, neglects potential inter-individual differences in structure-function relationships. Hence, although the exact same anatomical brain region along the IPS was targeted in each participant, the revealed inter-individual differences in the 
functional "hot spot" of task-related brain activity were neglected. This might have resulted in a slightly sub optimal stimulation in some of the participants, leading to a reduced effect size of the TMS-induced behavioural changes. However, as the power analysis revealed, this approach would have resulted in a significant behavioural effect when increasing the sample size from 5 to 9 participants.

When applying TMS based on the group Talairach coordinates, the TMSinduced behavioural effect sizes were also slightly reduced, both in comparison to the individual fMRI-guided as well as the individual MRI-guided Neuronavigation approaches. This "probabilistic" approach (Paus, 1999; Paus et al., 1997) uses frameless stereotaxy in order to navigate the TMS coil to specific standardised brain coordinates, e.g., Talairach coordinates that have been reported in the literature to be functionally activated during the execution of the respective task. Although this approach considers not only structural but also functional imaging data of the respective cognitive task, it still neglects the unique individual structure-function relationship by assuming perfect consistency across individuals in the location of task-related "activations" in standardised stereotaxic space. In our study, the revealed large inter-individual variance in task-related functional parietal activity changes considerably hampered the accuracy of this approach. As a consequence, the TMS-induced behavioural effect sizes during this TMS positioning approach were again smaller as compared to the individual imaging-guided results. However, as the power analysis revealed, this approach would also have resulted in a significant behavioural effect when increasing the sample size to 13 participants.

Finally, while the revealed inter-individual variance of the actual TMS target stimulation site during the individual fMRI-guided and MRI-guided TMS Neuronavigation approach represent the useful and important consideration of brain and taskrelevant inter-individual differences, the large inter-individual variance of the actual parietal target region during the P4 stimulation approach was mainly caused by the oversimplified assumption of inter-subject consistency between scalp landmarks and underlying anatomical brain regions. This anatomical landmark approach neither accounted for inter-individual differences in the functional architecture of the brain, i.e., inter-individual differences in structure-function relationships, nor for the existing inter-individual differences in the actual correspondence between specific scalp landmarks and underlying brain anatomy (Herwig, Satrapi, \& SchönfeldtLecuona, 2003; Okamoto et al., 2004). Although the 10-20 EEG system considers differences in absolute head sizes between participants by measuring the relative distances between individual cranial landmarks, inter-individual asymmetries and deformities of the skull and brain still cause considerable inaccuracy of electrode positioning (Habib, 1984; Myslobodsky \& Bar-Ziv, 1989; Towle et al., 1993). Accordingly, Herwig et al. (2003) evaluated and quantified several electrode positions and found interindividual variations concerning the actual correspondence to underlying anatomical brain regions. Here, our cognitive TMS study indeed showed that during this TMS coil positioning approach the revealed TMS-induced behavioural effect sizes were the smallest as compared to the other approaches. In fact, the conducted power analysis revealed that in order to still reveal a significant behav- 
ioural effect despite the inaccurate coil positioning, 47 participants would need to be tested.

Our findings clearly document a graded difference between the four TMS coil positioning approaches with the individual fMRI-guided TMS Neuronavigation yielding the strongest and the P4 stimulation approach yielding the smallest behavioural effect size. While all four approaches account for different aspects and different levels of brain- and task-related inter-individual variances, some still either underestimated (probabilistic approach) or overestimated (P4 approach) the variability in the location of the SCE that is present between individuals. In this respect, our findings suggest that in case of cognitive studies or during structure-function mappings of so-called "silent" brain regions, the optimal method, where possible, is to use TMS on the basis of individual functional brain imaging coordinates.

However, some words of caution are required. Although individually fMRI guided TMS is the most accurate method, our power calculations show that structural MRI guidance or mean Talairach coordinates with frameless stereotaxy are sufficient if the group size is large enough. Moreover, we investigated the functional relevance of one particular region of the IPS for one cognitive function and the extent to which our findings can be applied to other studies is uncertain. In fact, given the particularly large inter-individual variability along the IPS, (Zilles, Eickhoff, \& Palomero-Gallagher, 2003), the particular cognitive function investigated in this study may have biased our findings by specifically emphasising the advantages of an individual fMRI-guided TMS Neuronavigation as the only approach which explicitly accounts for these inter-individual differences in the functional architecture of the brain. It may be that for other brain regions or other cognitive tasks, the inter-individual differences in brain anatomy and structure-function relationships are less pronounced, thus reducing the difference between fMRI guided, MRI guided and probabilistic approaches making these latter two approaches an acceptable compromise between stimulation accuracy and pre-experimental effort. However, one can be confident that individual fMRI-guided TMS will be the most accurate and that $10-20$ system co-ordinates will have least power in cognitive studies.

It is also important to note that the question of determining the optimal TMS stimulation approach during cognitive studies is not sufficiently addressed by merely focusing on positioning the coil above the respective target stimulation site. In addition to the differential influence of different TMS coil positioning approaches as described above, different coil rotations above the exact same target stimulation site similarly influence the revealed behavioural effect sizes of TMS. In a recent study, Kammer, Vorwerg and Herrnberger (2007) used TMS to stimulate the visual cortex and investigated the dependency of phosphene thresholds on TMS current orientation. Importantly, with the aim of an advanced TMS neuronavigation system which allows storing the exact position and orientation of the TMS coil relative to an individual 3D anatomical MR scan, the authors were able to empirically relate the individual neuroanatomy in terms of site and orientation of the stimulated gyrus in the occipital cortex to the optimal current direction of TMS. They revealed that the optimal current orientation runs perpendicular to the underlying gyrus. Based on these findings, Kammer and colleagues (2007) conclude that similar to the motor 
system, the visual system behaves anisotropically when depolarised by TMS, and since this anisotropy might very well be a general feature all over the cortex, TMS coil orientation should always be considered when stimulating any cortical region. In addition, inter-individual differences in scalp-to-cortex distance requires consideration of individually-dosed TMS intensities as the exact same target site and coil orientation might still result in different TMS-induced electric field strengths between participants with varying absolute distances between the TMS coil and the respective target stimulation site (Knecht, Sommer, Deppe, \& Steinstrater, 2005).

Finally, in a series of studies, Wagner and colleagues (2008) demonstrated the need to account for the effects of the specific electric characteristics of cortical tissue. The authors evaluated the induced current density in a realistic model of the human head when being exposed to a time varying magnetic field. The tissue electric properties were varied to ascertain their influence on the induced currents. Interestingly, current density components normal to the tissue interface were shown to exist in all solutions within the cortex, contrary to the predictions of models that rely on symmetrical geometries. Additionally, modifications in the cortical geometry were shown to perturb the field and current density distributions in magnitude, location, and orientation such that the site of activation could be considerably altered, particularly in the case of, e.g., electrical and anatomical changes following a stroke (Wagner et al., 2006), or brain atrophy (Wagner et al., 2008).

In light of these and our own findings, future software developments are still needed in order to simultaneously account for all of these influencing factors. In this respect, an idealised TMS coil positioning approach would thus be based on individual fMRI data of a given participant, using a frameless stereotaxic system which allows online and real-time TMS neuronavigation to a specific target stimulation site while at the same time enabling real-time determination of the optimal target TMS coil orientation, online-dosing of the optimal TMS intensity based on individual scalp-to-target distance measurements, as well as online and real-time modelling of the induced current density distributions and their interaction with the specific tissue electric properties as present at the specific TMS target stimulation site.

\section{ACKNOWLEDGEMENTS}

A. T. S. and T. S. were supported by a grant from the Netherlands Organization for Scientific Research (NWO; grants 452-06-003 and 400-04-215). We thank our medical supervisor Cees van Leeuwen and our independent physician Martin van Boxtel. 


\section{REFERENCES}

Andoh, J., Artiges, E., Pallier, C., Rivière, D., Mangin, J. F., Cachia, A., et al. (2006). Modulation of language areas with functional MR image-guided magnetic stimulation. NeuroImage, 29(2), 619627.

Aron, A. R., Robbins, T. W., \& Poldrack, R. A. (2004). Inhibition and the right inferior frontal cortex. Trends in Cognitive Sciences, 8(4), 170-177.

Boroojerdi, B., Foltys, H., Krings, T., Spetzger, U., Thron, A., \& Topper, R. (1999). Localization of the motor hand area using transcranial magnetic stimulation and functional magnetic resonance imaging. Clinical Neurophysiology, 110(4), 699-704.

Boynton, G. M., Engel, S. A., Glover, G. H., \& Heeger, D. J. (1996). Linear Systems Analysis of Functional Magnetic Resonance Imaging in Human V1. Journal of Neuroscience, 16(13), 4207-4221.

Cohen, J. (1988). Statistical power analysis for the behavioral sciences (2 ed.). Hillsdale, NJ.

Cohen Kadosh, R., Cohen Kadosh, K., Schuhmann, T., Kaas, A., Goebel, R., Henik, A., et al. (2007). Virtual Dyscalculia Induced by Parietal-Lobe TMS Impairs Automatic Magnitude Processing. Current Biology, 17(8), 689-693.

Cohen Kadosh, R., Lammertyn, J., \& Izard, V. (2008). Are numbers special? An overview of chronometric, neuroimaging, developmental and comparative studies of magnitude representation. Progress in Neurobiology, 84(2), 132-147.

Critchley, H. D., Tang, J., Glaser, D., Butterworth, B., \& Dolan, R. J. (2005). Anterior cingulate activity during error and autonomic response. NeuroImage, 27(4), 885-895.

Di Lazzaro, V., Oliviero, A., Pilato, F., Saturno, E., Dileone, M., Mazzone, P., et al. (2004). The physiological basis of transcranial motor cortex stimulation in conscious humans. Clinical Neurophysiology, 115(2), 255-266.

Epstein, C. M., Verson, R., \& Zangaladze, A. (1996). Magnetic coil suppression of visual perception at an extracalcarine site. Journal of Clinical Neurophysiology, 13(3), 247-252.

Epstein, C. M., \& Zangaladze, A. (1996). Magnetic coil suppression of extrafoveal visual perception using disappearance targets. Journal of Clinical Neurophysiology, 13(3), 242-246.

Ettinger, G. J., Leventon, M. E., Grimson, W. E. L., Kikinis, R., Gugino, L., Cote, W., et al. (1998). Experimentation with a transcranial magnetic stimulation system for functional brain mapping. Medical Image Analysis, 2(2), 133-142.

Feredoes, E., Tononi, G., \& Postle, B. R. (2007). The Neural Bases of the Short-Term Storage of Verbal Information Are Anatomically Variable across Individuals. Jornal of Neuroscience, 27(41), 1100311008.

Gugino, L. D., Rafael Romero, J., Aglio, L., Titone, D., Ramirez, M., Pascual-Leone, A., et al. (2001). Transcranial magnetic stimulation coregistered with MRI: a comparison of a guided versus blind stimulation technique and its effect on evoked compound muscle action potentials. Clinical Neurophysiology, 112(10), 1781-1792.

Habib, M. (1984). CT assessment of right-left asymmetries in the human cerebral cortex. Journal of computer assisted tomography, 8(5), 922-922.

Hallett, M. (2000). Transcranial magnetic stimulation and the human brain. Nature, 406(6792), 147150.

Henik, A. (1982). Is three greater than five: The relation between physical and semantic size in comparison tasks. Memory \& Cognition, 10(4), 389-389.

Herwig, U., Kölbel, K., Wunderlich, A. P., Thielscher, A., von Tiesenhausen, C., Spitzer, M., et al. (2002). Spatial congruence of neuronavigated transcranial magnetic stimulation and functional neuroimaging. Clinical Neurophysiology, 113(4), 462-468.

Herwig, U., Satrapi, P., \& Schönfeldt-Lecuona, C. (2003). Using the International 10-20 EEG System for Positioning of Transcranial Magnetic Stimulation. Brain Topography, 16(2), 95-99.

Jasper, H. H. (1958). Appendix to report to Committee on Clinical Examination in EEG: the ten-twenty electrode system of the International Federation. Electroencephalography and Clinical Neurophysiology, 10, 371-371.

Kammer, T., Vorwerg, M., \& Herrnberger, B. (2007). Anisotropy in the visual cortex investigated by neuronavigated transcranial magnetic stimulation. NeuroImage, 36(2), 313-321. 
Kaufmann, L., Koppelstaetter, F., Delazer, M., Siedentopf, C., Rhomberg, P., Golaszewski, S., et al. (2005). Neural correlates of distance and congruity effects in a numerical Stroop task: an eventrelated fMRI study. NeuroImage, 25(3), 888-898.

Knecht, S., Sommer, J., Deppe, M., \& Steinstrater, O. (2005). Scalp position and efficacy of transcranial magnetic stimulation. Clinical Neurophysiology, 116(8), 1988-1993.

Krings, T., Buchbinder, B. R., Butler, W. E., Chiappa, K. H., Jiang, H. J., Cosgrove, G. R., et al. (1997). Functional magnetic resonance imaging and transcranial magnetic stimulation: Complementary approaches in the evaluation of cortical motor function. Neurology, 48(5), 1406-1416.

Myslobodsky, M. S., \& Bar-Ziv, J. (1989). Locations of occipital EEG electrodes verified by computed tomography. Electroencephalography and Clinical Neurophysiology, 72(4), 362-366.

Okamoto, M., Dan, H., Sakamoto, K., Takeo, K., Shimizu, K., Kohno, S., et al. (2004). Threedimensional probabilistic anatomical cranio-cerebral correlation via the international 10-20 system oriented for transcranial functional brain mapping. NeuroImage, 21(1), 99-111.

Pascual-Leone, A., Bartres-Faz, D., \& Keenan, J. P. (1999). Transcranial magnetic stimulation: studying the brain-behaviour relationship by induction of 'virtual lesions'. Philosophical Transactions of the Royal Society of London. Series B: Biological Sciences, 354(1387), 1229-1238.

Pascual-Leone, A., Walsh, V., \& Rothwell, J. (2000). Transcranial magnetic stimulation in cognitive neuroscience - virtual lesion, chronometry, and functional connectivity. Current Opinion in Neurobiology, 10(2), 232-237.

Paus, T. (1999). Imaging the brain before, during, and after transcranial magnetic stimulation. Neuropsychologia, 37(2), 219-224.

Paus, T., Jech, R., Thompson, C. J., Comeau, R., Peters, T., \& Evans, A. C. (1997). Transcranial Magnetic Stimulation during Positron Emission Tomography: A New Method for Studying Connectivity of the Human Cerebral Cortex. Journal of Neuroscience, 17(9), 3178-3184.

Penfield, W. (1958). Some mechanisms of consciousness discovered during electrical stimulation of the brain. Proceedings of the National Academy of Sciences of the United States of America, 44(2), 5151.

Picard, N., \& Strick, P. L. (2001). Imaging the premotor areas. Current Opinion in Neurobiology, 11(6), 663-672.

Pinel, P., Piazza, M., Le Bihan, D., \& Dehaene, S. (2004). Distributed and Overlapping Cerebral Representations of Number, Size, and Luminance during Comparative Judgments. Neuron, 41(6), 983-993.

Robertson, E. M., Théoret, H., \& Pascual-Leone, A. (2003). Studies in Cognition: The Problems Solved and Created by Transcranial Magnetic Stimulation. Journal of Cognitive Neuroscience, 15(7), 948960.

Rushworth, M. F. S., Walton, M. E., Kennerley, S. W., \& Bannerman, D. M. (2004). Action sets and decisions in the medial frontal cortex. Trends in Cognitive Sciences, 8(9), 410-417.

Sack, A. T. (2006). Transcranial magnetic stimulation, causal structure-function mapping and networks of functional relevance. Current Opinion in Neurobiology, 16(5), 593-599.

Sack, A. T., Kohler, A., Linden, D. E., Goebel, R., \& Muckli, L. (2006). The temporal characteristics of motion processing in hMT/V5+: combining fMRI and neuronavigated TMS. Neuroimage, 29(4), 1326-1335.

Sparing, R., Buelte, D., Meister, I. G., Pauscaron, T., \& Fink, G. R. (2008). Transcranial magnetic stimulation and the challenge of coil placement: A comparison of conventional and stereotaxic neuronavigational strategies. Human Brain Mapping, 29(1), 82-96.

Stewart, L., Battelli, L., Walsh, V., \& Cowey, A. (1999). Motion perception and perceptual learning studied by magnetic stimulation. Electroencephalography and Clinical Neurophysiology Suppl, 51, 334-350.

Talairach, J., \& Tournoux, P. (1988). Co-planar stereotaxic atlas of the human brain: New York: Thieme.

Thiel, A., Haupt, W. F., Habedank, B., Winhuisen, L., Herholz, K., Kessler, J., et al. (2005). Neuroimaging-guided rTMS of the left inferior frontal gyrus interferes with repetition priming. Neuroimage, 25(3), 815-823.

Towle, V. L., Bolaños, J., Suarez, D., Tan, K., Grzeszczuk, R., Levin, D. N., et al. (1993). The spatial location of EEG electrodes: locating the best-fitting sphere relative to cortical anatomy. Electroencephalography and Clinical Neurophysiology, 86(1), 1-6. 
Tzelgov, J. (1992). Automatic and intentional processing of numerical information. Journal of Experimental Psychology. Learning, memory, and cognition, 18(1), 166-166.

Wagner, T., Eden, U., Fregni, F., Valero-Cabre, A., Ramos-Estebanez, C., Pronio-Stelluto, V., et al. (2008). Transcranial magnetic stimulation and brain atrophy: a computer-based human brain model study. Experimental Brain Research, 186(4), 539-550.

Wagner, T., Fregni, F., Eden, U., Ramos-Estebanez, C., Grodzinsky, A., Zahn, M., et al. (2006). Transcranial magnetic stimulation and stroke: a computer-based human model study. Neuroimage, 30(3), 857-870.

Walsh, V., \& Pascual-Leone, A. (2003). Transcranial magnetic stimulation: A neurochronometrics of mind. Massachusetts: MIT Press.

Ziemann, U. (2004). TMS induced plasticity in human cortex. Reviews in the Neurosciences, 15(4), 253-266.

Zilles, K., Eickhoff, S., \& Palomero-Gallagher, N. (2003). The human parietal cortex: a novel approach to its architectonic mapping. Advances in Neurology, 93, 1-21. 



\section{CHAPTER 3}

\section{THE TEMPORAL CHARACTERISTICS OF FUNCTIONAL ACTIVATION IN BROCA'S AREA DURING OVERT PICTURE NAMING ${ }^{1}$}

\footnotetext{
${ }^{1}$ Based on: Schuhmann, T., Schiller, N.O, Goebel, R., \& Sack, A.T. (2009). The temporal characteristics of Broca's area during overt picture naming. Cortex, 45, 1111-1116.
} 


\begin{abstract}
The opercular and triangular sections of the inferior frontal gyrus, also known as Broca's area, have been shown to be involved in various language tasks. In the current study, we investigated both the functional role as well as the precise temporal involvement of Broca's area during language production, as taking place during picture naming. We applied online event-related TMS to Broca's area at five different time points after picture presentation, aiming to cover the complete language production process. Applying real TMS at $300 \mathrm{~ms}$ after picture presentation lead to an increase in picture naming latency, whereas sham stimulation and real stimulation at earlier and later time windows did not result in any changes in reaction time. Our methodological approach enabled us to get insight into the temporal characteristics of the involvement of this brain area during picture naming. Making use of this information and directly relating it to psycholinguistic models, we conclude that Broca's area may be involved in the process of syllabification during overt speech production.
\end{abstract}




\section{INTRODUCTION}

Our ability to communicate and comprehend verbal language represents one of the most crucial and complex human cognitive skills. By employing single case lesion studies, Broca (1861) and Wernicke (1874) pioneered in the attempt to directly relate different aspects of language processing to the proper functioning of distinct regions of the cerebral cortex. With the advent of modern functional brain imaging techniques, a systematic non-invasive investigation of the neural correlates of language became available also in healthy volunteers. Until today, a vast amount of studies have continued to reveal detailed insights into the exact functional neuroanatomy underlying speech production, using electrophysiological (MEG (Salmelin, 2007) and EEG (Schiller, Bles, \& Jansma, 2003; Schmitt, Münte, \& Kutas, 2000)) and metabolic brain imaging techniques (see for example Binder et al., 1997; Gernsbacher \& Kaschak, 2003). Other studies have utilised transcranial magnetic stimulation (TMS) to transiently disrupt neural processing, thereby effectuating a quantifiable change in behavioural performance when applying it to an area functionally involved in the task at question (Pascual-Leone, Walsh, \& Rothwell, 2000). These studies contributed to the understanding of the functional relevance of language-related brain areas to the behavioural execution of various language tasks (Andoh et al., 2006; Devlin, Matthews, \& Rushworth, 2003; Mottaghy et al., 1999; Mottaghy, Sparing, \& Topper, 2006; Naeser et al., 2005; Nixon, Lazarova, Hodinott-Hill, Gough, \& Passingham, 2004; Sakai, Noguchi, Takeuchi, \& Watanabe, 2002; Shapiro, Pascual-Leone, Mottaghy, Gangitano, \& Caramazza, 2001). However, only few of these TMS studies employed the advantage of event-related TMS to track the exact time point of functionally relevant neural activity during language production. Moreover, some neglected the high inter-individual variance in the anatomy of participants' brains. They applied TMS to sites that were defined by anatomical landmarks of the skull or standardised group coordinates, instead of making use of individual anatomical brain mapping.

Here, we investigated both the functional role as well as the precise temporal involvement of Broca's area during language production, as taking place during picture naming. Broca's area is an anatomically complex region including the pars opercularis (F3Op), the pars triangularis (F3Tr), and the pars orbitalis, which show anatomical variability between participants. We used individual anatomical mapping and frameless stereotaxy to precisely map and target Broca's area in each individual participant. We applied online event-related triple-pulse TMS (tpTMS), thus while participants were performing a picture naming task and at various time points after picture presentation. This enabled us to not only receive information about whether Broca's area is functionally relevant during picture naming, but to also exactly identify at which precise point in time the neural activity in Broca's area contributes to the processes of picture naming (Sack \& Linden, 2003; Walsh \& Pascual-Leone, 2003). Event related tpTMS was shown to be a good compromise between achieving sufficient temporal resolution and induced behavioural effect in higher cognitive functions (Sack, Camprodon, Pascual-Leone, \& Goebel, 2005). To 
control for effects caused by other than the direct magnetic stimulation of the brain, we included sham stimulation.

\section{METHODS}

\section{PARTICIPANTS}

Twelve healthy volunteers (5 men; mean age 23.2 years, standard deviation 2.08, range 20-26) participated in the study. All participants were native Dutch speakers, had normal or corrected-to-normal vision and had no history of neurological or psychiatric disorders. They received medical approval for participation and gave their written informed consent after being introduced to the procedure. The study was approved by the local Medical Ethical Commission.

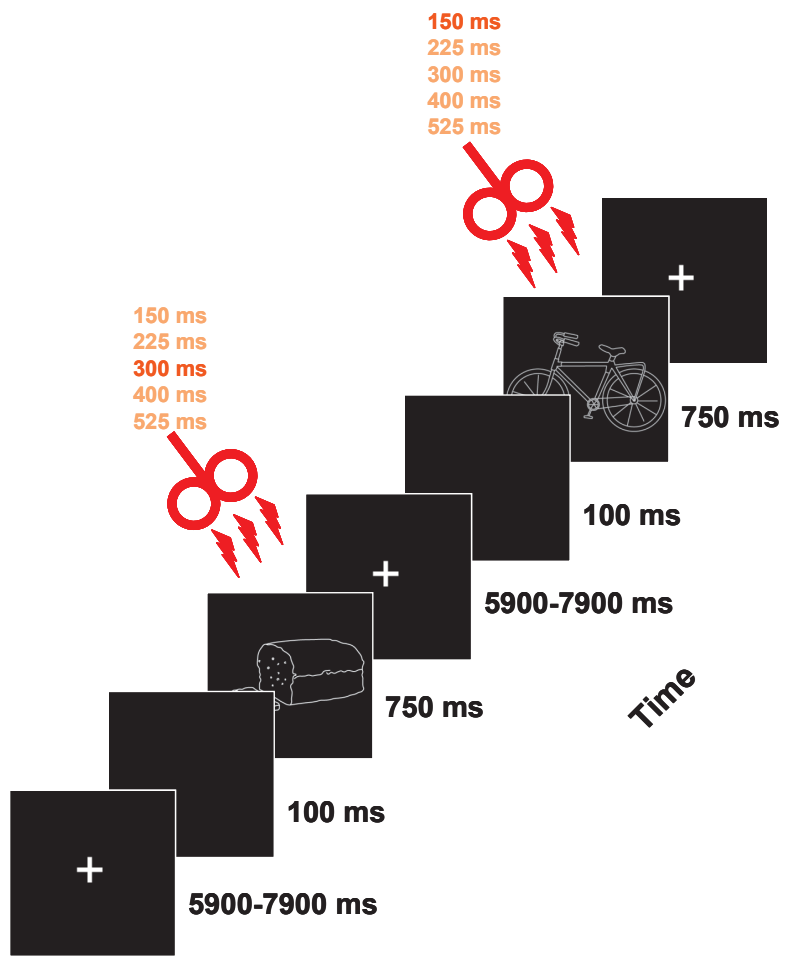

Figure 1. Experimental paradigm.

A trial consisted of the presentation of the fixation cross followed by a black screen, followed by the presentation of the picture. After picture presentation, tpTMS was applied randomly at one out of 5 different time windows. In the first trial shown in the figure, the TMS pulses were applied $300 \mathrm{~ms}$ after picture presentation and $150 \mathrm{~ms}$ after picture presentation in the second trial shown. Participants were instructed to name the presented picture as quickly as possible by responding into a microphone. After a jittered delay between 6-8 seconds, a new trial began. 


\section{OVERALL STUDY DESIGN}

We combined the methods of structural MRI and neuronavigated TMS to study the influence of a controlled neural activity disruption of Broca's area on picture naming latencies. Participants were tested in three separate sessions. In the first session, we obtained anatomical measurements of all participants using MRI. In the second and third session, participants were either stimulated with triple pulse real TMS or with triple pulse sham TMS, respectively, while performing a behaviourally controlled picture naming task. This study design and methodological approach enabled us to first define the target brain area based on the individual anatomical data and to subsequently neuronavigate the TMS coil to the anatomically defined stimulation site in each participant. The MRI-guided TMS neuronavigation was monitored online throughout the experiment, allowing for a precise determination of the actual stimulation site during task execution.

\section{STIMULI, PARADIGM AND PROCEDURE}

A set of ten simple white-on-black line drawings was used as target pictures. All items corresponded to monomorphemic, monosyllabic Dutch nouns. They were taken from the picture database of the Max Planck Institute for Psycholinguistics in Nijmegen, The Netherlands. All picture names had a length between three and five segments (phonemes). Each picture had a mean frequency of occurrence between 10 and 73 per million as determined by CELEX (Baayen, Piepenbrock, \& Gulikers, 1995), i.e., all pictures were of moderate frequency. The drawings were presented on a computer screen in front of the participant. The stimuli subtended a visual angle of $2.82^{\circ} \times 4.57^{\circ}$ and were displayed in the centre of the monitor. Each trial consisted of a fixation cross presented between 5,900 and $7,900 \mathrm{~ms}$, followed by a black screen for $100 \mathrm{~ms}$. Thereafter, one of the pictures was presented for $750 \mathrm{~ms}$. Participants were instructed to name the presented picture as quickly as possible by responding into a microphone. After a jittered delay between 6-8 seconds, a new trial began (see Figure 1).

\section{RESPONSE TIME ANALYSIS}

The entire experiment was recorded with a microphone positioned on the table in front of the participant. Acoustic information was digitised with the digital audio editing software GoldWave v 5.17 (GoldWave, New Foundland, Canada) with a sampling rate of $44 \mathrm{kHz}$. Prior to determining the speech onset, the acoustic signal was filtered to reduce noise. The latency of the verbal responses (reaction time) was measured on the screen using speech wave envelopes (see Figure 2). 


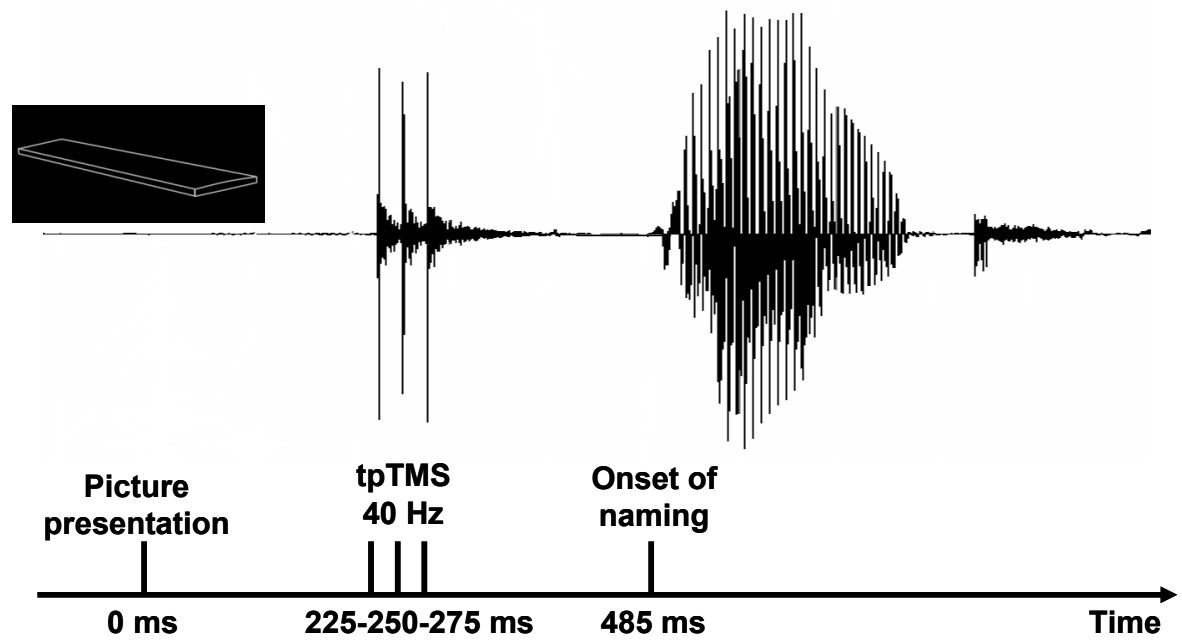

Figure 2. Trial-by-trial naming latency analysis.

The latency of the verbal responses (reaction time) was measured on the screen using speech wave envelopes. Onset of naming was defined as the first detectable amplitude in the digitised speechwaves.

\section{MRI MEASUREMENTS}

A high-resolution anatomical image was obtained from each participant in a 3-T magnetic resonance scanner (Siemens Allegra MR Tomograph; Siemens AG, Erlangen, Germany) at the Faculty of Psychology, Maastricht University, The Netherlands. The T1-weighted data set was acquired with the help of a magnetisationprepared rapid acquisition gradient echo (MPRAGE) sequence or a T1-weighted structural scan with an isotropic resolution of $1 \mathrm{~mm}$ using a modified driven equilibrium Fourier transform (MDEFT) sequence with optimised contrast for grey matter and white matter and imaging parameters.

\section{Cortical-surface reconstruction}

Data were analysed using the BrainVoyager QX 1.8 software package (BrainInnovation, Maastricht, The Netherlands). The high-resolution anatomical recordings were used for surface reconstruction of the left hemisphere of each participant. The surface reconstruction was performed in order to recover the exact spatial structure of the cortical sheet and to improve the visualisation of the anatomical gyrification.

\section{Co-registration of stereotaxic and MRI data}

For a precise positioning of the stimulation coil, we made use of the BrainVoyager TMS Neuronavigator (BrainInnovation, Maastricht, The Netherlands). This frameless stereotaxic system allows for an online individual navigation of a TMS coil above a specific anatomical area of the brain, as well as an imaging-guided navigation of the TMS coil to functionally defined brain regions-of-interest (see Sack, Kohler, Linden, Goebel, \& Muckli, 2006). 


\section{TMS MEASUREMENTS}

\section{TMS apparatus and stimulation parameters}

Biphasic TMS pulses were applied using the MagProX100 stimulator (Medtronic Functional Diagnostics A/S, Sklovunde, Denmark) and a figure-of-eight coil (MC$B 70$, inner radius $10 \mathrm{~mm}$, outer radius $50 \mathrm{~mm}$ ). The maximum output of this coil and stimulator combination is approximately 1.9 Tesla and $150 \mathrm{~A} / \mu \mathrm{S}$. A specific figure-of-eight placebo coil (MC-P-B70) was also employed in order to reproduce the same acoustic stimulation as the active coil while not inducing the magnetic field (sham stimulation). The coils were manually held tangentially to the skull with the coil handle oriented perpendicular to the opercular part of the inferior frontal gyrus using the online visualisation function of the BrainVoyager TMS Neuronavigator. Event-related tpTMS was applied with an inter-pulse-interval of $25 \mathrm{~ms}(40 \mathrm{~Hz})$ at $120 \%$ motor threshold.

\section{TMS localisation}

Broca's area is typically defined in terms of the pars opercularis and pars triangularis of the inferior frontal gyrus, represented in Brodmann's (Brodmann, 1909) cytoarchitectonic map as areas 44 and 45 . We targeted the pars opercularis of the inferior frontal gyrus (thus the posterior part of Broca's area) for stimulation. The stimulation site was localised using frameless stereotaxy (TMS neuronavigation (Cohen Kadosh et al., 2007; Sack, Kohler, Linden, Goebel, \& Muckli, 2006)) for both real and sham stimulation. Using such a TMS neuronavigation system enabled us to account for inter-individual differences in anatomical brain structures while stimulating. The average stimulation site coordinates in Talairach space were: $x=-49 ; y=13 ; z=26$.

\section{TMS procedure}

Individual motor thresholds (MTs) were determined as the intensity at which the stimulation of the left motor cortex with single-pulse TMS resulted in a visible movement of the contralateral thumb in $50 \%$ of the trials. The motor thresholds of the participants ranged from $27 \%$ to $42 \%$ of maximum stimulator output (mean = $35.40 \%(51.6 \mathrm{~A} / \mu \mathrm{S}), \mathrm{SD}=4.6)$. The mean stimulation intensity was set at $120 \%$ of the MT and therefore resulted in $42.5 \%(63.75 \mathrm{~A} / \mu \mathrm{S})$ of maximum stimulator output (range $33 \%$ to $50 \%$, SD $=5.3$ ). Throughout the entire experiment, participants were wearing earplugs to protect their ears from the clicking sound and to minimise the interference of the sounds during the task.

Participants were tested with event-related triple-pulse TMS in two separate sessions. In one session, participants were stimulated with real TMS, in the other session with sham TMS. The sequence of stimulation type was counterbalanced across participants. Prior to starting with the experiment, participants were familiarised with the stimuli and practised naming the stimuli repeatedly to reach a stable performance level in naming latency. Each experimental session consisted of 60 trials, divided into 4 blocks of fifteen trials each. Stimuli were presented and pulses were triggered using the software package "Presentation" (http://nbs.neurobs.com). 
Event-related tpTMS was applied at five different points in time following picture presentation onset, namely at 1) $150-175-200 \mathrm{~ms}, 2) 225-250-275 \mathrm{~ms}, 3$ ) $300-$ 325-350 ms, 4) 400-425-450 ms, and 5) 525-550-575 ms. In a sixth condition, no TMS pulses were applied during the trial. This latter condition served as an online baseline measurement. The presentation of the pictures and the TMS time window conditions were fully randomised across trials within each session.

\section{RESULTS}

Two participants did not go through the entire experimental TMS session since they experienced discomfort due to strong contractions of face muscles and were therefore excluded from the analysis. Incorrect trials of the remaining ten participants were excluded from the naming latency analysis. Incorrect trials (errors) were defined as semantically incorrect responses, hesitations, and extremely delayed responses (responses taking longer than $2000 \mathrm{~ms}$ ).

The effect of TMS on Broca's area was tested at the above-mentioned five time intervals. The same set of intervals was also tested applying sham TMS. To correct for the positive skew of the response times distribution, a logarithmic transformation of the data was performed. Moreover, response times that were above or below 2 standard deviations of the mean were defined as outliers and excluded from the naming latency analysis.

\section{TMS-INDUCED CHANGES IN PICTURE NAMING ACCURACY}

The mean error rates were calculated for each time window for both the sham and real stimulation. The mean amount of errors during the real TMS experiment ranged from $.01(\mathrm{SD}=.03)$ up to $.05(\mathrm{SD}=.07)$ in the different time windows. During the sham TMS experiment, the mean amount of errors was comparable, ranging from $.01(S D=.04)$ to $.04(S D=.07)$. A two-factorial ANOVA with stimulation type (real TMS versus sham TMS) and time window (no stimulation, stimulation at $150,225,300,400$, and $525 \mathrm{~ms}$ after stimulus presentation) as the two within-subject factors did not reveal a significant effect of time window $(F(5,45)=$ $.769 ; p=.577)$ nor stimulation type $(F(1,9)=1.569 ; p=.242)$. Moreover, there was no interaction between stimulation type and time window $(F(5,45)=1.058 ; p$ $=.396)$, revealing that the error rate not only did not differ between time windows within one stimulation type, but that the time windows did also not differ between the two stimulation types.

\section{TMS-INDUCED CHANGES IN PICTURE NAMING LATENCY}

The mean reaction times (RTs) were calculated for each time window for sham and real stimulation. As can be seen in Figure 3, during the sham TMS condition, mean RTs did not vary considerably at any of the time points of stimulation. In fact, the RTs during sham stimulation for every time window (TMS at 150: $453 \mathrm{~ms}$, SD = 44.08; TMS at 225: $453 \mathrm{~ms}, \mathrm{SD}=32.91$; TMS at 300: $461 \mathrm{~ms}, \mathrm{SD}=32.64$; TMS at 400: $466 \mathrm{~ms}, \mathrm{SD}=29.89$; TMS at 525: $457 \mathrm{~ms}, \mathrm{SD}=39.31$ ) were almost iden- 
tical to the mean RT revealed without TMS pulses (449 ms; SD $=41.22$ ). This implies that applying no TMS and sham TMS at various time intervals after picture onset similarly do not result in behavioural changes in picture naming.

In contrast, real TMS over Broca's area did increase the reaction times during picture naming compared to no TMS ( $454 \mathrm{~ms}$; SD $=43.89$ ). The RTs increase by approximately $15 \mathrm{~ms}$ for the time windows at $150 \mathrm{~ms}(470 \mathrm{~ms}, \mathrm{SD}=45.88)$ and $225 \mathrm{~ms}(471 \mathrm{~ms}, \mathrm{SD}=43.19)$. In contrast, the time window of $300 \mathrm{~ms}$ was characterised by a large effect of TMS on reaction times of approximately $50 \mathrm{~ms}$ ( $505 \mathrm{~ms}$, $\mathrm{SD}=75.02$ ) compared to no TMS. At 400 and $525 \mathrm{~ms}$, however, RTs rapidly decreased again and went back to baseline (no TMS) level. This indicates that real TMS over Broca's area has a very time-specific effect on picture naming, only interfering with behaviour when applying tpTMS at $300 \mathrm{~ms}$ after the onset of picture presentation. In Figure 4, it can be seen that the reaction times do not differ between real and sham TMS in the no TMS condition, whereas the reaction times then slowly diverge, finding their peak difference in the time window of TMS at $300 \mathrm{ms,}$ and finally go back to the same level again in the last two time windows, namely at TMS at $400 \mathrm{~ms}$ and TMS at $525 \mathrm{~ms}$.

In order to test whether the time- and stimulation type-specific changes in reaction times also reached statistical significance, we entered the data into a twofactorial ANOVA. The two-factorial ANOVA with stimulation type (real TMS versus sham TMS) and time window (no stimulation, stimulation at 150, 225, 300, 400, and $525 \mathrm{~ms}$ after stimulus presentation) as the two within-subject factors revealed a main effect of time window $(F(5,45)=3.63 ; p<.01)$, indicating that the effect of TMS over Broca's area differed between the various time points of stimulation. The analysis did not reveal a main effect of stimulation type $(F(1,9)=.98 ; p=.348)$. Importantly, the analysis yielded a significant interaction of stimulation type and time window $(F(5,45)=4.22 ; p<.01)$, demonstrating that the time-specific effect of TMS differed between real and sham TMS. To break down the interaction, simple contrasts were performed comparing the different time windows between the stimulation types, using LSD correction. These revealed a significant effect of stimulation type only in the time window of $300 \mathrm{~ms}(F(1,9)=7.90 ; p<.05)$, while all other time windows showed no significant difference compared to baseline. Moreover, a significant interaction between stimulation type and time window was again only found in the time window of $300 \mathrm{~ms}(F(1,9)=7.52 ; \mathrm{p}<.05)$, while all time-points before and after 300 ms showed no significant difference between real and sham TMS.

This finding was further confirmed by separate one-factorial analyses of both real and sham TMS. Under sham stimulation, no difference between the time windows could be found $(F(5,45)=1.27, p=.293)$. Under real stimulation, however, a main effect of condition was revealed $(F(4,45)=5.55 ; p<.001)$. Simple contrasts were performed to compare the five conditions in which stimulation was applied at several time windows with the baseline condition in which no pulses were applied. This revealed a significant difference in RTs only for the condition in which stimulation took place at $300 \mathrm{~ms}$ after stimulus presentation $(F(1,9)=9.04 \mathrm{p}<.05)$. 


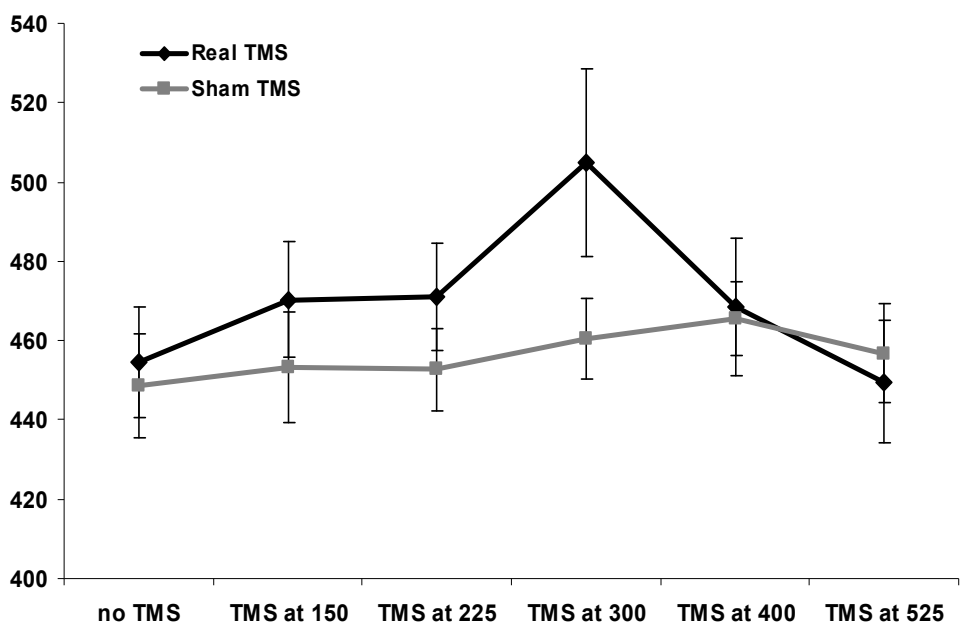

Figure 3. Behavioural results.

Mean reaction times (naming latencies) in ms (plus standard errors) for all time windows, separately shown for real (black line) versus sham (grey line) TMS. Only when applying real TMS at $300 \mathrm{~ms}$ after picture presentation, a significant increase in RTs was found.

\section{DISCUSSION}

Making use of neuronavigated event-related TMS, we were able to define the exact time period at which Broca's area is critically engaged in the process of picture naming. Applying online triple-pulse TMS over Broca's area resulted in an increase in RTs in a picture naming task only when applied $300 \mathrm{~ms}$ after picture presentation. In contrast, stimulating Broca's area at earlier time-windows (150 ms and 225 $\mathrm{ms}$ ) or at later time windows ( $400 \mathrm{~ms}$ and $525 \mathrm{~ms}$ ) did not have a significant effect on RTs. Sham stimulation over Broca's area did not affect RTs at any time. Our data thus confirm the relevance of Broca's area during picture naming as suggested by previous studies (see Vigneau et al., 2006 for an overview), and provides new insights into the exact spatio-temporal characteristics of its specific contribution during speech production.

The question arises which of the cognitive processes involved in speech production is disturbed at 300-350 ms after picture presentation. Based on chronometric behavioural and electrophysiological data, an estimation of the entire time course of speech production has been made (Indefrey \& Levelt, 2004). It is likely that accessing the lexical concept, i.e. the meaning of an object upon presenting a picture of that object takes about 150-200 ms (Schmitt, Munte, \& Kutas, 2000; Thorpe, Fize, \& Marlot, 1996). Retrieving the corresponding lexical entry or lemma from the mental lexicon takes on average another $75 \mathrm{~ms}$ (Glaser \& Dungelhoff, 1984; Schmitt, Munte, \& Kutas, 2000). Putting the order into shape, i.e. phonological encoding including segmental and metrical retrieval as well as syllabification can 
start about 80 ms later (Schmitt, Schiltz, Zaake, Kutas, \& Münte, 2001; Van Turennout, Hagoort, \& Brown, 1998). The process of syllabification of a word, i.e. the assignment of previously selected segments to syllable positions such as onset, nucleus, and coda, depends on the length of a word. Evidence from lateralised readiness potentials showed that syllabification of a word proceeds at a speed of approximately $25 \mathrm{~ms}$ per segment (Van Turennout, Hagoort, \& Brown, 1997). In other words, to syllabify a word consisting of five segments, such as "tafel" (table), takes about $125 \mathrm{~ms}$. The final step before articulation, namely phonetic encoding lasts about $145 \mathrm{~ms}$. Thus, the overall time to name an object from a picture takes between 550 and 600 ms on average (Levelt, Praamstra, Meyer, Helenius, \& Salmelin, 1998). However, since we only used monomorphemic, monosyllabic, and relatively familiar objects, and since naming these objects was well-trained, we obtained faster mean reaction times of approximately 470 ms. Presumably, shorter RTs reflect an equidistant shortening of all the processes involved in speech production. On the basis of that assumption, tpTMS at $300 \mathrm{~ms}$ after picture onset is likely to disturb Broca's area either in the process of syllabification. This interpretation is supported by Indefrey and Levelt's (2004) study. They performed a meta-analysis of the relevant imaging literature on word production combined with an analysis of data on time course activation and reported specific regions and time windows of activation for the core processes of word production, based on which they proposed an involvement of Broca's area in the process of syllabification. However, even though the interpretation of Broca's area being involved in the process of syllabification is compatible with our findings, it still remains speculative and follow-up studies are needed to provide further evidence with respect to this interpretation.

Also note that this interpretation is framed within the theoretical assumptions made in Levelt's (1999) discrete serial view of word production. The reason this theoretical framework was selected for the current study is that it is one of the most explicit models making precise predictions which can be tested empirically. However, we would like to mention that there are other approaches to word production as well, including interactive activation theories (i.e. Dell, 1986). In Dell's theory, strictly speaking all levels of word encoding, e.g. the conceptual level, the lexical level, the morphological level and the phonological level, are activated simultaneously. Therefore, interactive activation theories would not predict a specific time window for the process of e.g. syllabification.

\section{ACKNOWLEDGEMENTS}

T.S., N.O.S., and A.T.S. were supported by grants from the Netherlands Organisation for Scientific Research (NWO; grant number 452-06-003, 400-04-215, and 453-02-006). We thank our medical supervisor Cees van Leeuwen, and our independent physician Martin van Boxtel. 


\section{REFERENCES}

Andoh, J., Artiges, E., Pallier, C., Riviere, D., Mangin, J. F., Cachia, A., et al. (2006). Modulation of language areas with functional MR image-guided magnetic stimulation. Neuroimage, 29(2), 619627.

Binder, J. R., Frost, J. A., Hammeke, T. A., Cox, R. W., Rao, S. M., \& Prieto, T. (1997). Human brain language areas identified by functional magnetic resonance imaging. Journal of Neuroscience, $17(1), 353-362$.

Broca, P. (1861). Remarques sur le siège de la faculté du langage articule, suivies d'une observation d'aphémie (Perte de la Parole). Bulletin de la Société Anatomique de Paris, 6, 330-357.

Brodmann, K. (1909). Vergleichende Lokalisationslehre der Großhirnrinde in ihren Prinzipien dargestellt auf Grund des Zellenbaues: Leipzig: Johann Ambrosius Barth.

Cohen Kadosh, R., Cohen Kadosh, K., Schuhmann, T., Kaas, A., Goebel, R., Henik, A., et al. (2007). Virtual Dyscalculia Induced by Parietal-Lobe TMS Impairs Automatic Magnitude Processing. Current Biology, 17(8), 689-693.

Dell, G. S. (1986). A spreading-activation theory of retrieval in sentence production. Psychol Rev, 93(3), 283-321.

Devlin, J. T., Matthews, P. M., \& Rushworth, M. F. (2003). Semantic processing in the left inferior prefrontal cortex: a combined functional magnetic resonance imaging and transcranial magnetic stimulation study. Journal of Cognitive Neuroscience, 15(1), 71-84.

Gernsbacher, M. A., \& Kaschak, M. P. (2003). Neuroimaging studies of language production and comprehension. Annual Review of Psychology, 54, 91-114.

Glaser, W. R., \& Dungelhoff, F. J. (1984). The time course of picture-word interference. Journal of Experimental Psychology. Human preception and Performance, 10(5), 640-654.

Indefrey, P., \& Levelt, W. J. (2004). The spatial and temporal signatures of word production components. Cognition, 92(1-2), 101-144.

Levelt, W. J., Praamstra, P., Meyer, A. S., Helenius, P., \& Salmelin, R. (1998). An MEG study of picture naming. Journal of Cognitive Neuroscience, 10(5), 553-567.

Levelt, W. J., Roelofs, A., \& Meyer, A. S. (1999). A theory of lexical access in speech production. The Behavioral and Brain Sciences, 22(1), 1-38; discussion 38-75.

Mottaghy, F. M., Hungs, M., Brugmann, M., Sparing, R., Boroojerdi, B., Foltys, H., et al. (1999). Facilitation of picture naming after repetitive transcranial magnetic stimulation. Neurology, 53(8), 1806-1812.

Mottaghy, F. M., Sparing, R., \& Topper, R. (2006). Enhancing picture naming with transcranial magnetic stimulation. Behavioral Neurology, 17(3-4), 177-186.

Naeser, M. A., Martin, P. I., Nicholas, M., Baker, E. H., Seekins, H., Kobayashi, M., et al. (2005). Improved picture naming in chronic aphasia after TMS to part of right Broca's area: an openprotocol study. Brain and Language, 93(1), 95-105.

Nixon, P., Lazarova, J., Hodinott-Hill, I., Gough, P., \& Passingham, R. (2004). The inferior frontal gyrus and phonological processing: an investigation using rTMS. Journal of Cognitive Neuroscience, 16(2), 289-300.

Pascual-Leone, A., Walsh, V., \& Rothwell, J. (2000). Transcranial magnetic stimulation in cognitive neuroscience--virtual lesion, chronometry, and functional connectivity. Current Opinion in Neurobiology, 10(2), 232-237.

Sack, A. T., Camprodon, J. A., Pascual-Leone, A., \& Goebel, R. (2005). The dynamics of interhemispheric compensatory processes in mental imagery. Science, 308(5722), 702-704.

Sack, A. T., Kohler, A., Linden, D. E., Goebel, R., \& Muckli, L. (2006). The temporal characteristics of motion processing in hMT/V5+: combining fMRI and neuronavigated TMS. Neuroimage, 29(4), 1326-1335.

Sack, A. T., \& Linden, D. E. (2003). Combining transcranial magnetic stimulation and functional imaging in cognitive brain research: possibilities and limitations. Brain Research. Cognitive Brain Research, 43(1), 41-56.

Sakai, K. L., Noguchi, Y., Takeuchi, T., \& Watanabe, E. (2002). Selective priming of syntactic processing by event-related transcranial magnetic stimulation of Broca's area. Neuron, 35(6), 1177-1182. 
Salmelin, R. (2007). Clinical neurophysiology of language: the MEG approach. Clinical Neurophysiology, 118(2), 237-254.

Schiller, N. O., Bles, M., \& Jansma, B. M. (2003). Tracking the time course of phonological encoding in speech production: an event-related brain potential study. Brain Research. Cognitive Brain Research, 17(3), 819-831.

Schmitt, B. M., Munte, T. F., \& Kutas, M. (2000). Electrophysiological estimates of the time course of semantic and phonological encoding during implicit picture naming. Psychophysiology, 37(4), 473484.

Schmitt, B. M., Münte, T. F., \& Kutas, M. (2000). Electrophysiological estimates of the time course of semantic and phonological encoding during implicit picture naming. Psychophysiology, 37(4), 473484.

Schmitt, B. M., Schiltz, K., Zaake, W., Kutas, M., \& Münte, T. F. (2001). An electrophysiological analysis of the time course of conceptual and syntactic encoding during tacit picture naming. Journal of Cognitive Neuroscience, 13(4), 510-522.

Shapiro, K. A., Pascual-Leone, A., Mottaghy, F. M., Gangitano, M., \& Caramazza, A. (2001). Grammatical distinctions in the left frontal cortex. Journal of Cognitive Neuroscience, 13(6), 713720.

Thorpe, S., Fize, D., \& Marlot, C. (1996). Speed of processing in the human visual system. Nature, 381(6582), 520-522.

Van Turennout, M., Hagoort, P., \& Brown, C. M. (1997). Electrophysiological evidence on the time course of semantic and phonological processes in speech production. Journal of Experimental Psychology. Learning, Memory, and Cognition, 23(4), 787-806.

Van Turennout, M., Hagoort, P., \& Brown, C. M. (1998). Brain activity during speaking: from syntax to phonology in 40 milliseconds. Science, 280(5363), 572-574.

Vigneau, M., Beaucousin, V., Herve, P. Y., Duffau, H., Crivello, F., Houde, O., et al. (2006). Metaanalyzing left hemisphere language areas: phonology, semantics, and sentence processing. Neuroimage, 30(4), 1414-1432.

Walsh, V., \& Pascual-Leone, A. (2003). Neurochronometrics of Mind: Transcranial magnetic stimulation in Cognitive Science. Cambridge, MA: MIT Press.

Wernicke, C. (1874). Der aphasische Symptomencomplex, eine physiologische Studie auf anatomischer Basis. Breslau: Cohn \& Weigert. 



\section{CHAPTER 4}

\section{SPEAKING OF WHICH: DISSECTING THE NEUROCOGNITIVE NETWORK OF LANGUAGE PRODUCTION ${ }^{1}$}

${ }^{1}$ Based on: Schuhmann, T., Schiller, N. O., Goebel, R., \& Sack, A. T. (submitted). Speaking of which: Dissecting the neurocognitive network of language production. 


\begin{abstract}
Speaking is the most important human skill for communication. Yet, the precise neurocognitive mechanisms underlying language production are far from being understood. We probed the behavioural relevance of Broca's area, Wernicke's area, and MTG for successful language production by directly interfering with their local neural activity changes online, i.e., during overt picture naming, using MRI-guided chronometric TMS. This enabled us to test whether neural activity in these regions is functionally relevant for picture naming and to chart the exact time point at which this neural activity is critical. We demonstrate that the midsection of the left MTG becomes functionally relevant first at $225 \mathrm{~ms}$ post-picture-onset, followed by Broca's area at $300 \mathrm{~ms}$, and Wernicke's area at $400 \mathrm{~ms}$. Interestingly, during this late time window, MTG shows a second peak of functional relevance. Each area thus contributed at different stages during the speech production process, suggesting distinct underlying functional roles within this complex, multi-componential skill. We propose that the first peak of functional relevance in MTG represents the early process of word retrieval. This information is then processed in Broca's area for subsequent phonological encoding, which in turn back-projects the phonologically encoded word to MTG, resulting in its second peak of functional relevance. At the same time, the retrieved information is forward-projected to Wernicke's area for internal self-monitoring. We thus propose a neurobiological model of speech production according to which successful speaking relies on intact, spatiotemporally specific feed-forward and recurrent feedback loops within a left-hemispheric frontotemporal brain network.
\end{abstract}




\section{INTRODUCTION}

Speaking is one of the most complex cognitive skills. A seemingly simple task like naming an object requires the coordination of a series of processes such as the selection of meanings, the retrieval of words, syntactic and phonological encoding, and articulation. The time course of these processes is described in models of speech production based on speech error data (Caramazza, 1997; Dell et al., 1997) and/or chronometric behavioural studies (Levelt et al., 1999).

More recently, imaging studies aimed at identifying which brain areas underlie the process of speaking. Based on a meta-analysis of 82 neuroimaging studies Indefrey and Levelt (2004) suggested a detailed description of both the time course and location of cerebral activations during speech production. According to their neurocognitive model of speech production, the mid section of the left MTG is functionally relevant first for lexical retrieval, followed by Wernicke's area for phonological code retrieval, and finally Broca's area for syllabification. The model, although influential in psycholinguistics, is still debated in the field of cognitive neuroscience, and so far no empirical brain research study has yet directly tested these spatiotemporal network predictions within controlled experimental conditions.

Here, we apply MRI-guided chronometric transcranial magnetic stimulation (TMS) online, i.e., during overt picture naming in order to identify the functional relevance and spatiotemporal dynamics underlying speaking on an individual brainsystem level. Applying chronometric TMS over several distinct MRI-identified language regions in the left hemisphere, namely the posterior part of the superior temporal gyrus (STG), which is part of Wernicke's area, the inferior frontal gyrus (IFG), which is part of Broca's area, and the midsection of the left middle temporal gyrus (MTG), enabled us to unravel the precise temporal involvement of these different areas within the language brain network. We applied MRI-guided TMS while participants were performing a picture naming task (online) and at various time points between picture onset and overt speech production (at 150ms, 225ms, $300 \mathrm{~ms}, 400 \mathrm{~ms}$ and $525 \mathrm{~ms}$ after picture presentation). This permitted us to not only receive information about whether these areas are functionally relevant during the picture naming task, but also to exactly identify at which precise point(s) in time the neural activity in these areas functionally contributed to the process of speech production.

Based on the speech production model presented by Indefrey and Levelt (2004), we hypothesised that posterior STG, IFG, and the midsection of the left MTG, all represent functionally relevant nodes of the effective brain connectivity network underlying speech production. We therefore expected that TMS to each of these regions should significantly interfere with successful overt picture naming (Hypothesis 1). We moreover hypothesised that each region has a distinct functional role and contributes at specific stages during the complex, multicomponential process of speech production. Therefore, neural activation in these three brain regions should be functionally relevant at distinct time points during picture naming (Hypothesis 2). 


\section{METHODS}

\section{PARTICIPANTS}

Twelve healthy volunteers ( 5 men; mean age 23.2 years, standard deviation 2.08 , ranging from 20 to 26 years) participated in the study. All participants were native Dutch speakers, had normal or corrected-to-normal vision and had no history of neurological or psychiatric disorders. They received medical approval for participation and gave their written informed consent after being introduced to the procedure. The study was approved by the local Medical Ethical Commission.

\section{OVERALL STUDY DESIGN}

Participants were tested in four separate sessions. In the first session, we obtained anatomical brain measurements of all participants using MRI. We performed a surface reconstruction to recover the spatial structure of the cortical sheet based on the white-grey-matter boundary. We then identified three prominent languagerelated areas in the left hemisphere, namely IFG (Broca's area), posterior STG (Wernicke's area), and the midsection of the left middle temporal gyrus (MTG), on the basis of each individual brain gyrification. In the following three sessions, we used frameless stereotaxy for MRI-guided TMS neuronavigation to these target regions, and applied triple-pulse chronometric TMS during the execution of a behaviourally-controlled picture naming task in order to study the influence of a controlled neural activity disruption on picture naming latencies. The order of stimulation site was randomised. This study design and methodological approach enabled us to first define the target brain area based on the individual anatomical data and to subsequently neuronavigate the TMS coil to the anatomically defined stimulation sites in each participant. The MRI-guided TMS neuronavigation was monitored online throughout the experiment, allowing for a precise determination of the actual stimulation site during task execution.

\section{STIMULI, PARADIGM AND PROCEDURE}

A set of ten simple white-on-black line drawings was used as target pictures. All items corresponded to monomorphemic, monosyllabic Dutch nouns. They were taken from the picture database of the Max Planck Institute for Psycholinguistics in Nijmegen, The Netherlands. All picture names had a length between three and five segments (phonemes). Each picture had a mean frequency of occurrence between 10 and 73 per million as determined by CELEX (Baayen, Piepenbrock, \& Gulikers, 1995), i.e., all target picture names were of moderate frequency.

The drawings were presented on a computer screen in front of the participant. The stimuli subtended a visual angle of $2.82^{\circ} \times 4.57^{\circ}$ and were displayed in the centre of the monitor on a black background. Each trial consisted of a fixation cross presented between 5900 and 7900 ms, followed by a black screen for 100 ms. Thereafter, one of the pictures was presented for $750 \mathrm{~ms}$. Participants were instructed to name the presented picture as quickly as possible by responding into a 
microphone. After a jittered delay between 6-8 seconds, the next trial began (see Figure 1).

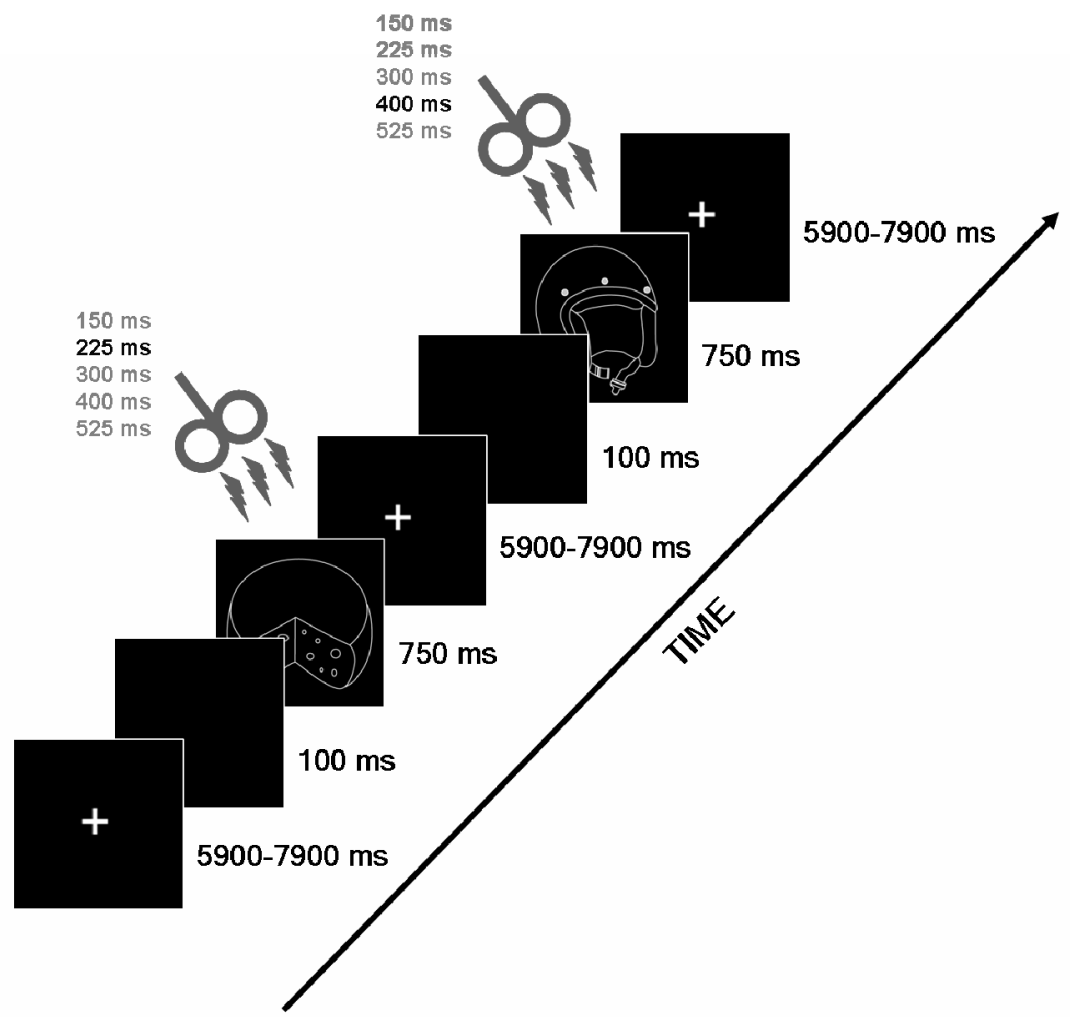

Figure 1. Experimental paradigm.

A trial consisted of the presentation of the fixation cross followed by a black screen, followed by the presentation of the picture. After picture presentation, triple pulse erTMS was applied randomly at one out of 5 different time windows. In the first trial shown in the figure, the TMS pulses were applied 225 $\mathrm{ms}$ after picture presentation and in the second trial shown, at $400 \mathrm{~ms}$ after picture presentation. Participants were instructed to name the presented picture as quickly as possible by responding into a microphone. After a jittered delay between 6-8 seconds, a new trial began.

\section{RESPONSE TIME ANALYSIS}

The entire experiment was recorded with a microphone positioned on the table in front of the participant. Acoustic information was digitised with the digital audio editing software GoldWave v 5.17 (GoldWave, New Foundland, Canada) with a sampling rate of $44 \mathrm{kHz}$. Prior to determining the speech onset, the acoustic signal was filtered to reduce noise. The latency of the verbal responses (reaction time) was measured on the screen using speech wave envelopes (see Figure 2). 

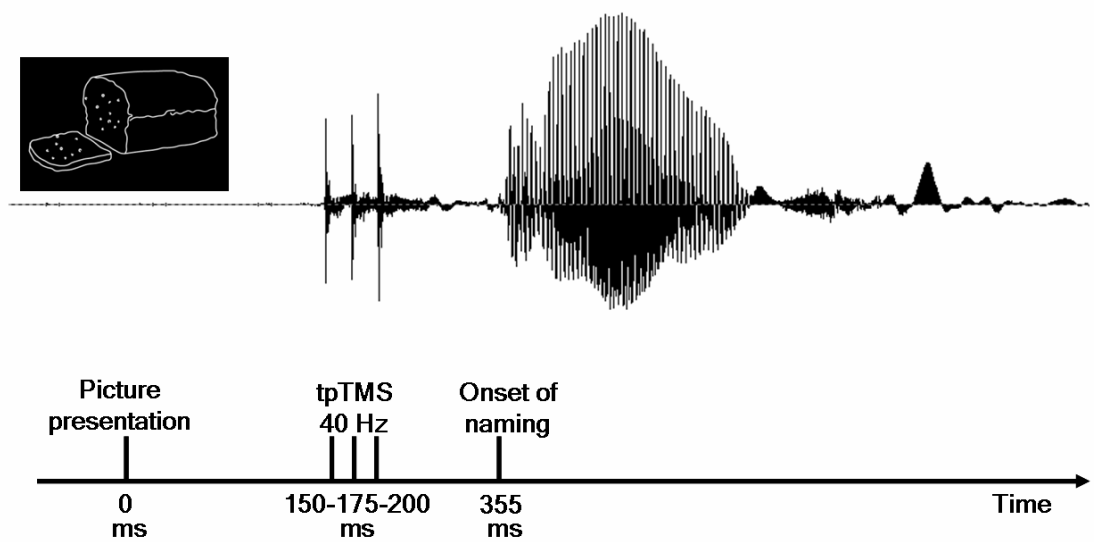

Figure 2. Trial-by-trial naming latency analysis.

The latency of the verbal responses (RT) was measured on the screen using speech wave envelopes. Onset of naming was defined as the first detectable amplitude in the digitised speech-waves.

\section{MRI MEASUREMENTS}

A high-resolution anatomical image was obtained from each participant in a 3-T magnetic resonance scanner (Siemens Allegra MR Tomograph; Siemens AG, Erlangen, Germany) at the Faculty of Psychology and Neuroscience, Maastricht University, The Netherlands. The T1-weighted data set was acquired with the help of a magnetisation-prepared rapid acquisition gradient echo (MPRAGE) sequence or a T1-weighted structural scan with an isotropic resolution of $1 \mathrm{~mm}$ using a modified driven equilibrium Fourier transform (MDEFT) sequence with optimised contrast for GM and WM and imaging parameters.

Cortical-surface reconstruction

Data were analysed using the BrainVoyager QX 1.8 software package (BrainInnovation, Maastricht, The Netherlands). The high-resolution anatomical recordings were used for surface reconstruction of the left hemisphere of each participant (Kriegeskorte and Goebel, 2001). The surface reconstruction was performed in order to recover the exact spatial structure of the cortical sheet and to improve the visualisation of the anatomical gyrification. The white-grey-matter boundary was segmented with a region growing method preceded by inhomogeneity correction of signal intensity across space. The borders of the two resulting segmented subvolumes were tessellated to produce a surface reconstruction of the left hemisphere.

Co-registration of stereotaxic and MRI data

For a precise positioning of the stimulation coil, we made use of the BrainVoyager TMS Neuronavigator (BrainInnovation, Maastricht, The Netherlands). This neuro- 
navigator system consists of several miniature ultrasound transmitters which are attached to a participant's head, as well as the TMS coil. The ultrasound markers continuously transmit ultrasonic pulses to a receiving sensor device. The measurement of the relative spatial position of these transmitters in 3-D space is based on travel time of the transmitted ultrasonic pulses to three microphones of the receiving sensor device. Local spatial coordinate systems are created by linking the relative raw spatial position of the ultrasound senders to a set of fixed additional landmarks on the participant's head. The specification of these fixed landmarks is achieved via a digitising pen that also hosts two transmitting ultrasound markers in order to measure its relative position in 3-D space. The three anatomical landmarks we used to define the local coordinate system were the nasion and the two incisurae intertragicae. The neuronavigation system then provides topographic information of the head ultrasound transmitters relative to a participant-based coordinate frame. Similarly, the TMS coil also hosts a set of ultrasound transmitters whose relative spatial positions are linked to fixed landmarks specified on the coil in order to calculate another local coordinate system. After having defined the local spatial coordinate system for the participant's head and the TMS coil in real 3-D space, these coordinate systems have to be co-registered with the coordinate system of the MR space. For TMS-MRI co-registration, the same landmarks digitised on the participant's head are specified on the head reconstruction of the anatomical data from the MR sequence. After the landmarks specified on the real head have been co-registered with those on the reconstructed head, events occurring around the head of the participant in real space are registered online and visualised in realtime at correct positions relative to the participant's anatomical reconstruction of the brain. The TMS coil can now be neuronavigated to a specific anatomical area of each participant. TMS neuronavigation was based on data in AC-PC space (rotating the cerebrum into the anterior commissure - posterior commissure plane) in order to avoid any additional transformations that could distort the correspondence between MRI and stereotaxic points.

\section{TMS MEASUREMENTS}

TMS apparatus and stimulation parameters

Biphasic TMS pulses were applied using the MagProX100 stimulator (Medtronic Functional Diagnostics A/S, Sklovunde, Denmark) and a figure-of-eight coil (MC$B 70$, inner radius $10 \mathrm{~mm}$, outer radius $50 \mathrm{~mm}$ ). The maximum output of this coil and stimulator combination is approximately 1.9 Tesla and $150 \mathrm{~A} / \mu \mathrm{S}$. The coil was manually held tangentially to the skull with the coil handle oriented perpendicular to the opercular part of the inferior frontal gyrus using the online visualisation function of the BrainVoyager TMS Neuronavigator. Chronometric triple-pulse TMS was applied with an inter-pulse-interval of $25 \mathrm{~ms}(40 \mathrm{~Hz})$ at $120 \%$ motor threshold.

\section{TMS localisation}

Broca's area is typically defined in terms of the pars opercularis and pars triangularis of the inferior frontal gyrus, corresponding to areas 44 and 45 in Brodmann's 
cytoarchitectonic map (Brodmann, 1909). We targeted the pars opercularis of the inferior frontal gyrus (thus the posterior part of Broca's area) for stimulation.

Wernicke's speech region extends over the left inferior parietal lobule. The core of this region occupies the left temporal plane behind the transverse gyrus of Heschl and the posterior part of the left superior temporal gyrus. For stimulation, we aimed for the posterior part of the left superior temporal gyrus. This can also be described as the posterior part of Brodmann area (BA) 22 (Brodmann, 1909).

The final region, according to Indefrey and Levelt (2004) responsible for conceptual preparation and lexical selection, was the mid section of the left middle temporal gyrus (MTG). This stimulation site, as well as IFG and posterior STG, was localised using the BrainVoyager TMS Neuronavigator. This enabled us to account for intra-individual differences in anatomical brain structures when stimulating. For precise localisation of target points on each individual participant, see Figure 3.

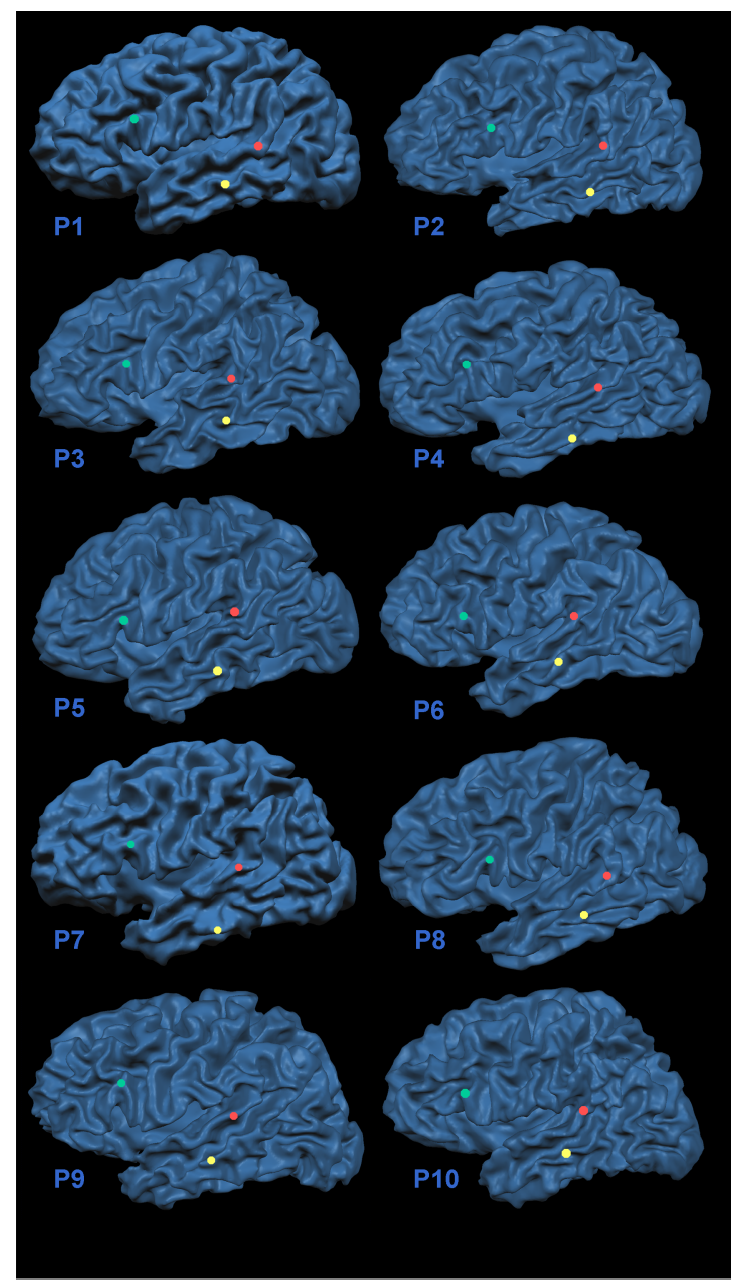

Figure 3. Localisation of target points for each individual participant.

Surface reconstruction based on the white-grey-matter boundary of each individual participant with target points of stimulation. IFG displayed in green, MTG displayed in yellow, STG displayed in red. Standardised mean coordinates in TAL space for each stimulation site were the following: IFG: $x=-49 ; y=13, z=26$; MTG: $x$ $=-57 ; y=-44 ; z=18$; STG: -55 ; $44 ; z=18$. 


\section{TMS procedure}

Individual motor thresholds (MTs) were determined as the intensity at which the stimulation of the left motor cortex with single-pulse TMS resulted in a visible movement of the contra-lateral thumb in $50 \%$ of the trials. The motor thresholds of the participants ranged from $27 \%$ to $42 \%$ of maximum stimulator output (mean = $35.40 \%[51.6 \mathrm{~A} / \mu \mathrm{S}], \mathrm{SD}=4.6)$. The mean stimulation intensity was set at $120 \%$ of the MT and therefore resulted in $42.5 \%(63.75 \mathrm{~A} / \mu \mathrm{S})$ of maximum stimulator output (range $33 \%$ to $50 \%$, SD $=5.3$ ). Throughout the entire experiment, participants were wearing earplugs to protect their ears from the clicking sound and to minimise the interference of the sounds during the task.

Participants were tested with chronometric triple-pulse TMS in three separate sessions. Prior to starting with the experiment, participants were familiarised with the stimuli and practiced naming the stimuli repeatedly to reach a stable performance level in naming latency. Each experimental session consisted of 60 trials, divided into 4 blocks of fifteen trials each. Stimuli were presented and pulses were triggered using the software package "Presentation" (http://nbs.neuro-bs.com).

Chronometric TMS was applied at five different points in time following picture presentation onset, namely at 1) $150-175-200 \mathrm{~ms}, 2) 225-250-275 \mathrm{~ms}, 3) 300-$ $325-350 \mathrm{~ms}$, 4) 400-425-450 ms, and 5) 525-550-575 ms. In a sixth condition, no TMS pulses were applied during the trial. This latter condition served as an online baseline measurement. The presentation of the pictures and the TMS time window conditions were fully randomised across trials within each session.

\section{Data analysis}

Two of the participants did not go through the entire experimental TMS session since they experienced discomfort due to strong contractions of face muscles and were therefore excluded from the analysis. Incorrect trials of the remaining ten participants were also excluded from the analysis. Incorrect trials (errors) were defined as semantically incorrect responses, hesitations, and extremely delayed responses (responses taking longer than $2000 \mathrm{~ms}$ ).

The effect of TMS on the three different areas was tested at the abovementioned five time intervals between stimulus onset and the TMS pulse, ranging from $150 \mathrm{~ms}$ to $525 \mathrm{~ms}$ following picture presentation. The RT data of the correct responses were further tested for normal distribution and variance homogeneity. These tests revealed that the RT data were positively skewed. In order to obtain a normal distribution, the entire data set underwent a logarithmic LN transformation. This ensured the suitability of the RT data for parametric statistical testing. Moreover, response times that were above or below 2 standard deviations of the mean were defined as outliers and were excluded from the analysis. 


\section{RESULTS}

\section{ERROR ANALYSIS}

Delayed responses and errors were analysed together. We performed a twofactorial repeated measures ANOVA with stimulation site and time window as within-subject factors on the error rate. Neither the main effect of site $(F=0.85 ; p$ $=0.44)$, nor the main effect of time window $(F=0.15 ; p=0.98)$ or the interaction of the two factors $(F=0.77 ; p=0.65)$ were significant, indicating that the amount of errors did not differ between stimulation sites and time windows.

\section{TMS-INDUCED CHANGES IN PICTURE NAMING LATENCY}

We analysed the picture naming latency data based on the full factorial model according to our experimental design, thus performing a two-factorial repeated measures ANOVA with stimulation site (IFG, MTG, STG) and time window (no stimulation, stimulation at $150,225,300,400$, and 525 ms after stimulus presentation) as the two within-subject factors. This analysis revealed no main effect of stimulation site $(F(2,18)=.86 ; p=.44)$, and a significant main effect of time window $(F(5,45)$ $=7.23 ; \mathrm{p}<.0001)$, indicating that the effect of TMS differed between the various time points of stimulation. Importantly, the analysis also revealed a significant interaction of stimulation site and time window $(F(10,90)=3.20 ; p<.01)$, showing that the time-specific effect of TMS differed between the stimulation sites. This significant interaction statistically supports the notion that the difference between the different time windows of TMS application is significantly different between stimulation sites, or in other words, that stimulation sites differ in their neural activity if functionally relevant for picture naming. Simple contrast analyses revealed that this interaction between both factors was mainly driven by the difference between sites in time window 225 ms, 300 ms, and 400 ms. Hence, the difference between sites in the temporal profile across all time windows is mainly on these three levels.

The interaction term was further investigated making use of separate onefactorial analyses of IFG, STG, and MTG stimulation sessions. When stimulating IFG, a main effect of time window was revealed $(F(4,45)=5.55 ; p<.001)$. Simple contrasts were performed to compare the five conditions in which stimulation was applied at different time windows, with the baseline condition in which no pulses were applied. This revealed a significant difference in response time only for the condition in which stimulation took place at $300 \mathrm{~ms}$ after stimulus presentation $(F(1,9)=9.04 ; p<0.05$; see Figure $4 A)$. When stimulating the MTG, also a main effect of time window was revealed $(F(4,45)=5.13 p<.01)$. Simple contrast analyses demonstrated a significant difference in reaction times between the no TMS condition and the time window of $225 \mathrm{~ms}(\mathrm{~F}(1,9)=1.77, \mathrm{p}<.01)$ and between the no TMS condition and the time window of $400 \mathrm{~ms}(F(1,9)=29.45 \mathrm{p}<$ .001 ; see Figure $4 \mathrm{~B}$ ). The one-factorial ANOVA of Wernicke's stimulation also revealed a main effect of time window $(F(5,45)=2.59 ; p<.05)$. Simple contrasts analyses showed that, compared to the no TMS condition, applying TMS only had a 
significant effect on reaction times in the time window of $400 \mathrm{~ms}(F(1,9)=13.6$; $\mathrm{p}$ $<.01$; see Figure $4 \mathrm{C}$ ).

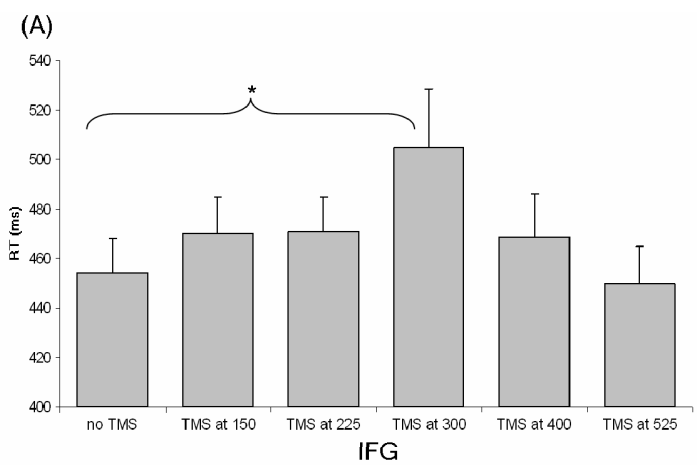

(B)

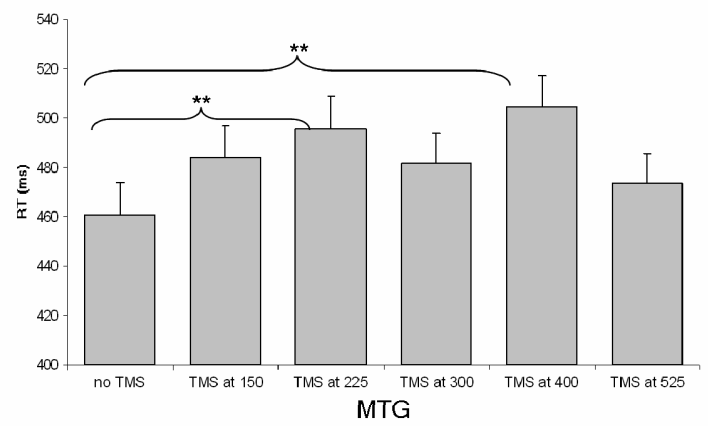

(C)

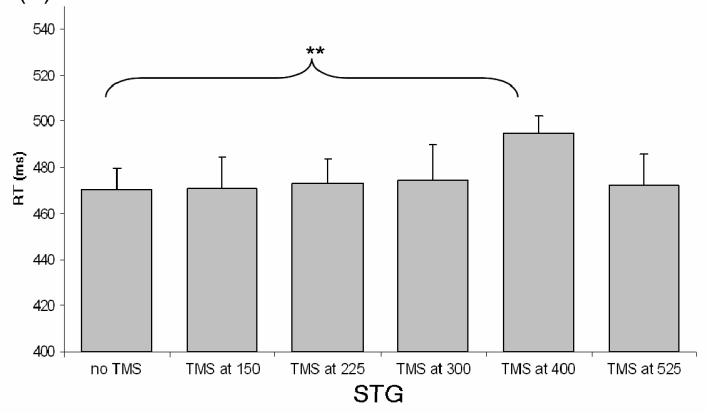

Figure 4. Mean RTs (naming latencies) in ms (plus standard errors) for all time windows.

Single asterisk indicate a significant difference of $p<0.05$, double asterisk indicate a significant difference of $p<0.001$. (A) TMS results of IFG interference. (B) TMS results of interference of the midsection of the left middle temporal gyrus. (C) TMS results of posterior STG interference. 


\section{DISCUSSION}

The current study provides direct empirical evidence for the functional relevance and temporal specificity of three prominent language-related areas in the left hemisphere, namely IFG (Broca's area), posterior STG (Wernicke's area), and the midsection of the left MTG, for successful speech production. By using individualised MRI-guided neuronavigated chronometric TMS over all three regions in an withinsubject design, we were able to show that, as suggested by current speech production theorists (e.g. Indefrey and Levelt, 2004), IFG, posterior STG and the midsection of the left MTG all represent functionally relevant nodes of a complex brain connectivity network underlying successful overt picture naming. This aspect is in clear agreement with Hypothesis 1 of our study, providing direct empirical evidence regarding the functionally relevant cognitive contribution of a widely distributed specific neurocognitive network underlying speech production. We moreover revealed that all three regions are functionally relevant at different points in time during speech production, suggesting a specific temporal order in which neural activity within these regions is critical for successful overt picture naming. This finding is again in general agreement with the idea of a specific spatiotemporal organisation within the speech production network in terms of relative time course and effective brain connectivity as proposed by speech production models (evidence in favour of Hypothesis 2). Concretely, we could show that left MTG is relevant at two distinct time points during picture naming, namely at an early stage, exactly as suggested by current modular speech production models, but also at a later stage again, as documented in our data by a second peak of functional relevance in left MTG. This second peak at around $400 \mathrm{~ms}$ temporally coincides with the functional relevance of posterior STG (Wernicke's area). IFG (Broca's area), on the other hand, seems to be functionally relevant between the early and late MTG activity, and thus slightly prior to the late functional relevance of posterior STG.

Although our study did reveal that all three brain areas are functionally relevant at different stages during the speech production process, as predicted by the neurocognitive model of speech production presented by Indefrey and Levelt (2004), our empirical data do not agree in all aspects with these predictions. According to the model, left MTG is functionally relevant first for lexical retrieval, which is indeed what we found. In contrast to our findings, however, the model also predicts that Wernicke's area is relevant prior to Broca's area, underlying the cognitive subprocess of phonological code retrieval. Moreover, no second (late) functional relevance of MTG as revealed in our study is discussed in current speech production models.

In the following, we would like to make an attempt in interpreting and integrating our empirical findings with the existing literature and different models of speech production in order to account for this partial discrepancy. Moreover, we would like to offer possible alternative (maybe additional) functional roles of Broca's area, Wernicke's area and MTG during speech production. It should be noted that it naturally becomes a matter of speculation and interpretation to post-hoc assign a specific functional role to each of the stimulated brain regions based on our empiri- 
cal findings (reverse-inference, (Poldrack, 2006) at this point, but we do believe that such a speculation is appropriate and useful.

According to the speech production model by Levelt and colleagues (Levelt et al., 1999), the first step in speech production planning is called conceptualisation. In this phase, the content of an utterance is represented as pre-linguistic units or concepts. During the next step, called formalisation, concepts become lexicalised, i.e. lexical entries corresponding to the concepts are retrieved from the mental lexicon. Formalisation can be divided into two separate processes, namely grammatical (or syntactic) and phonological encoding. During grammatical encoding, the syntactic structure of an utterance is specified. In contrast, during phonological encoding, the phonological form or sound of a word is specified (e.g., the phonemes or segments and the lexical stress) and so-called "phonological words" are created. After formalisation is completed, each phonological word has to be converted into a format that can be used to control neuromuscular commands necessary for the execution of articulatory-motor movements. The phonological word forms the basis for the retrieval of pre-compiled articulatory motor programs from a mental syllabary (Levelt and Wheeldon, 1994; Cholin et al., 2006). These motor programs may be represented in terms of gestural scores, which specify the relevant articulatory gestures and their timing. The final step includes the execution of these gestures by the articulatory apparatus, which results in overtly produced speech.

We propose that the early effect at $225 \mathrm{~ms}$ in left MTG could represent the early process of lexical retrieval during which concepts become lexicalised (Salmelin et al., 1994). Regarding the functional relevance of IFG at around 300 ms, we argued on the basis of the results of an earlier study that the process being disturbed at this time point is likely to be the process of syllabification (Schuhmann et al., 2008). However, although other TMS studies over IFG have similarly and consistently revealed its functional importance for speech production (Mottaghy et al., 1999; Shapiro et al., 2001; Sakai et al., 2002; Devlin et al., 2003; Nixon et al., 2004; Naeser et al., 2005; Andoh et al., 2006; Mottaghy et al., 2006), our current data, and especially the relative timing of the functional relevance of IFG with regard to posterior STG and the left MTG, makes the exact functional contribution of IFG during the process of overt picture naming less straightforward and clear-cut as previously thought.

Our findings may indicate the involvement of IFG in various aspects during speech production, including phonological, syntactic and semantic aspects. In accordance with this interpretation, recent studies suggest that respective subdivisions of IFG need to be considered which may constitute a functional segregation and contribution of IFG during speaking. According to Hagoort (2005), e.g., the inferior frontal gyrus 'binds' phonological, syntactic and semantic aspects with a function-location mapping from more posterior to anterior, respectively. Our data suggest that besides the mere involvement of Broca's area in retrieving precompiled articulatory motor programs from a mental syllabary (Levelt and Wheeldon, 1994; Cholin et al., 2006), some parts of Broca's area may also be involved in the process of phonological encoding, during which the phonological form or sound 
of a word is specified (e.g., the phonemes or segments and the lexical stress) or the phonetic encoding of these phonological segments, where fully syllabified socalled "phonological words" are created. Similar claims have been made by Friederici et al. (2009) and Schnur et al. (2009). Assuming that the early MTG effect at $225 \mathrm{~ms}$ represents lexical retrieval and the later effect at $300 \mathrm{~ms}$ observed in IFG the subsequent phonological encoding, the second peak of functional relevance found in left MTG and the peak in posterior STG at around $400 \mathrm{~ms}$ possibly indicate that the phonologically encoded concept may then be back-projected from IFG to the left MTG and at the same time also forward-projected to posterior STG. We propose that this feedback likely represents the neural connectivity mechanisms underlying internal speech monitoring (Hohenberger et al., 2002; Hohenberger and Keller, 2002; Leuninger et al., 2004; Christoffels et al., 2007; Schiller et al., 2009).

Most speakers produce numerous words per second, seemingly without effort or conscious control of the speaking process. Nevertheless, we constantly monitor our own speech output on aspects such as content, grammaticality, fluency and volume. Without monitoring, producing speech can potentially lead to embarrassment, for instance, when taboo words are uttered unintentionally (Motley et al., 1982) or speech output can result in awkward mishearing (Garnes and Bond, 1980). In word production, all critical sub-processes such as conceptual preparation, lexical and syntactic encoding, phonological encoding, and articulation (see Levelt et al., 1999) are likely to be subject to such internal monitoring mechanisms. According to one of the most influential models of speech production (Levelt et al., 1999), self-monitoring is a centrally controlled process with limited capacity, which evaluates the quality of the speech by means of the speech comprehension process. The speech comprehension system, used for understanding speech of others, also sub serves verbal external self-monitoring. In a similar vein, the abstract phonological code is presumably used for internal self-monitoring. Thus, it has been proposed that at the level of phonological encoding, potential speech production errors are controlled for via internal self-monitoring processes (see Levelt et al., 1999; Postma, 2000; Hartsuiker and Kolk, 2001) during which information is delivered to the speech comprehension system (posterior STG), where it is parsed and then transferred to the verbal monitor. The verbal monitor compares the parsed speech and the intentions of the speaker to the linguistic standards.

This interpretation is in line with the relative timing and feedback projections between IFG, left MTG, and posterior STG, as revealed in our study. We concretely identified an early effect in left MTG supposedly being the neural substrate underlying the process of lexical retrieval, after which neural information is sent forward to IFG for subsequent phonological encoding. IFG in turn back-projects the phonologically encoded concept to left MTG while at the same time forward-projecting it to posterior STG, thus the speech comprehension system, for internal self-monitoring purposes (see Figure 5). Studies documenting the existence of direct and effective white matter fiber connections between Broca's area and Wernicke's date back to 1895 when Dejerine (1895) defined the arcuate fasciculus (AF) as the prominent fiber tract connecting these two areas based on post-mortem dissections. More recently, diffusion tensor imaging studies empirically identified and described these 
white matter connections in the healthy living brain (Basser et al., 1994; Makris et al., 1997; Basser et al., 2000; Catani et al., 2002; Makris et al., 2005).

These studies indicate that the AF is not the only white matter tract connecting Broca's area and Wernicke's area, but that there are additional dorsal and ventral pathways. The dorsal pathway runs from Broca's area via the superior longitudinal fasciculus to posterior temporal lobe with connectivities to the lateral STG and MTG (Parker et al., 2005; Frey et al., 2008). Our data suggest that the existence of such white matter pathway connections between these prominent language-related brain regions such as IFG, MTG and posterior STG, might be of particular functional relevance during speech production and that the human language function is thus not only based on the grey matter of circumscribed brain regions in the frontal and the temporal cortex, but that instead, successful speech production largely depends on intact white matter fiber tracts connecting these adjacent as well as distant language-related brain regions.

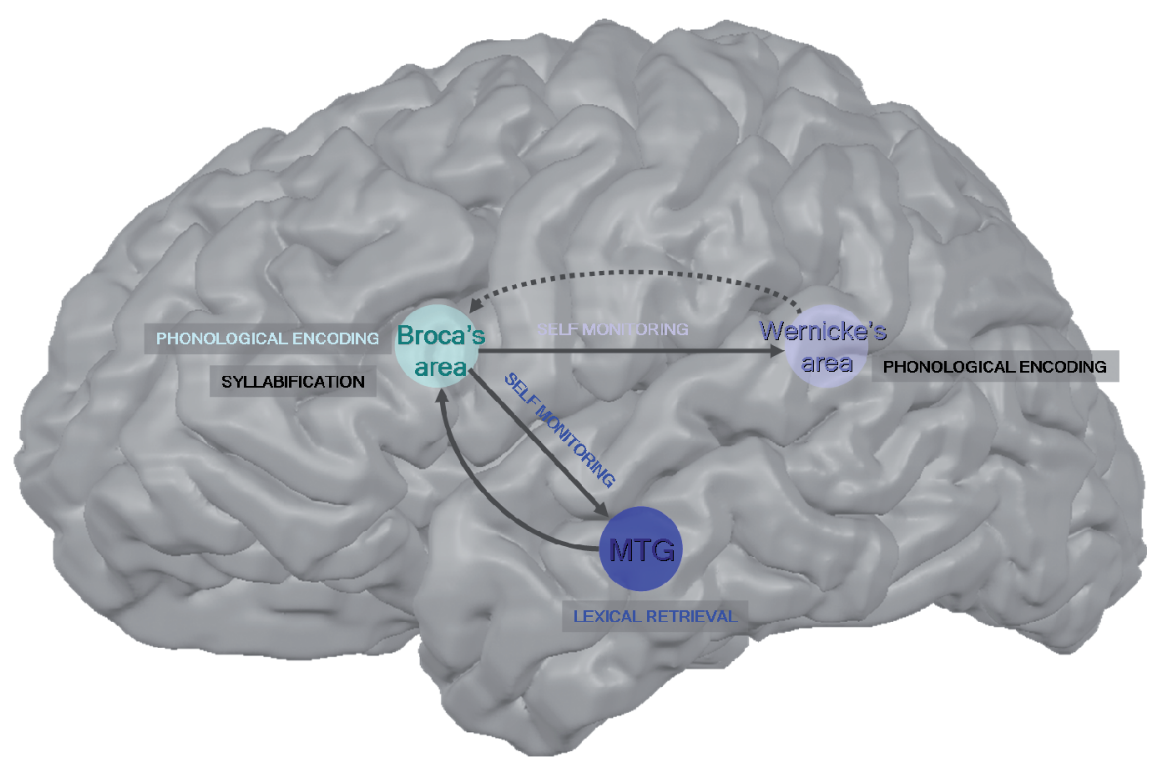

Figure 5. Proposed network of processing components and pathways involved in speech production.

During speaking, the midsection of the left middle temporal gyrus (MTG) becomes functionally relevant first (first peak at $225 \mathrm{~ms}$ ), underlying the process of lexical retrieval. From there, the neural information is sent forward to IFG for subsequent phonological encoding (300 ms). IFG in turn back-projects the phonologically encoded concept to left MTG (second peak at $400 \mathrm{~ms}$ ) while at the same time forward-projecting it to posterior STG for internal self-monitoring purposes (400 ms). posterior STG then back-projects the evaluated neural information to IFG in order to initiate the motor planning process (pathway indicated in dashed arrow; not backed up by our current data).

Color-coded fond = direct evidence revealed in current study design; black fond = evidence from other studies and/or general concepts of the speech production process. 


\section{REFERENCES}

Andoh, J., Artiges, E., Pallier, C., Riviere, D., Mangin, J. F., Cachia, A., et al. (2006). Modulation of language areas with functional MR image-guided magnetic stimulation. Neuroimage, 29(2), 619627.

Basser, P. J., Mattiello, J., \& LeBihan, D. (1994). MR diffusion tensor spectroscopy and imaging. Biophysical Journal, 66(1), 259-267.

Basser, P. J., Pajevic, S., Pierpaoli, C., Duda, J., \& Aldroubi, A. (2000). In vivo fiber tractography using DT-MRI data. Magnetic Resonance in Medicine, 44(4), 625-632.

Brodmann, K. (1909). Vergleichende Lokalisationslehre der Großhirnrinde in ihren Prinzipien dargestellt auf Grund des Zellenbaues: Leipzig: Johann Ambrosius Barth.

Caramazza, A. (1997). How many levels of processing are there in lexical access? Cognitive Neuropsychology, 14(1), 177-208.

Catani, M., Howard, R. J., Pajevic, S., \& Jones, D. K. (2002). Virtual in vivo interactive dissection of white matter fasciculi in the human brain. Neuroimage, 17(1), 77-94.

Cholin, J., Levelt, W. J., \& Schiller, N. O. (2006). Effects of syllable frequency in speech production. Cognition, 99(2), 205-235.

Christoffels, I. K., Formisano, E., \& Schiller, N. O. (2007). Neural correlates of verbal feedback processing: an fMRI study employing overt speech. Human Brain Mapping, 28(9), 868-879.

Dejerine, J. J. (1895). Anatomie des centres nerveux. Paris: Rueff et Cie.

Dell, G. S., Schwartz, M. F., Martin, N., Saffran, E. M., \& Gagnon, D. A. (1997). Lexical access in aphasic and nonaphasic speakers. Psychological Review, 104(4), 801-838.

Devlin, J. T., Matthews, P. M., \& Rushworth, M. F. (2003). Semantic processing in the left inferior prefrontal cortex: a combined functional magnetic resonance imaging and transcranial magnetic stimulation study. Journal of Cognitive Neuroscience, 15(1), 71-84.

Frey, S., Campbell, J. S., Pike, G. B., \& Petrides, M. (2008). Dissociating the human language pathways with high angular resolution diffusion fiber tractography. Journal of Neuroscience, 28(45), $11435-11444$.

Friederici, A. D. (2009). Pathways to language: fiber tracts in the human brain. Trends in Cognitive Science, 13(4), 175-181.

Garnes, S., \& Bond, Z. S. (1980). A slips of the ear? A snip of the ear? A slip of the year? In V. A. Fromkin (Ed.), Errors in Linguistic Performance: Slips of the Tongue, Ear, Pen and Hand (pp. 231239). New York: Academic Press.

Hagoort, P. (2005). On Broca, brain, and binding: a new framework. Trends in Cognitive Science, 9(9), 416-423.

Hartsuiker, R. J., \& Kolk, H. H. (2001). Error monitoring in speech production: a computational test of the perceptual loop theory. Cognitive Psychology, 42(2), 113-157.

Hohenberger, A., Happ, D., Leuninger, H., Meier, R. P., Cormier, K., \& Quinto-Pozos, D. (2002). Modality-dependent aspects of sign language production: Evidence from slips of the hands and their repairs in German Sign Language. In Modality and structure in signed and spoken languages. ( $\mathrm{pp}$. 112-142). New York, NY US: Cambridge University Press.

Hohenberger, A., \& Keller, J. (2002). On the amodal nature of the monitor: Sign vs. spoken language processing. Paper presented at the Conference Name|. Retrieved Access Date|. from URL|.

Indefrey, P., \& Levelt, W. J. (2004). The spatial and temporal signatures of word production components. Cognition, 92(1-2), 101-144.

Kriegeskorte, N., \& Goebel, R. (2001). An efficient algorithm for topologically correct segmentation of the cortical sheet in anatomical mr volumes. Neuroimage, 14(2), 329-346.

Leuninger, H., Hohenberger, A., Waleschkowski, E., Menges, E., \& Happ, D. (2004). The impact of modality on language production: Evidence from slips of the tongue and hand. In T. Pechman \& $\mathrm{C}$. Habel (Eds.), Multidisciplinary approaches to language production (pp. 219-277). Berlin: Mouton de Gruyter.

Levelt, W. J., Roelofs, A., \& Meyer, A. S. (1999). A theory of lexical access in speech production. The Behavioral and Brain Sciences, 22(1), 1-38; discussion 38-75.

Levelt, W. J., \& Wheeldon, L. (1994). Do speakers have access to a mental syllabary? Cognition, 50(13), 239-269. 
Makris, N., Kennedy, D. N., McInerney, S., Sorensen, A. G., Wang, R., Caviness, V. S., Jr., et al. (2005). Segmentation of subcomponents within the superior longitudinal fascicle in humans: a quantitative, in vivo, DT-MRI study. Cerebral Cortex, 15(6), 854-869.

Makris, N., Worth, A. J., Sorensen, A. G., Papadimitriou, G. M., Wu, O., Reese, T. G., et al. (1997). Morphometry of in vivo human white matter association pathways with diffusion-weighted magnetic resonance imaging. Annals of Neurology, 42(6), 951-962.

Motley, M. T., Camden, C. T., \& Baars, B. J. (1982). Covert formulation and editing of anomalies in speech production: Evidence from experimentally elicited slips of the tongue. Journal of Verbal Learning \& Verbal Behavior, 21(5), 578-594.

Mottaghy, F. M., Hungs, M., Brugmann, M., Sparing, R., Boroojerdi, B., Foltys, H., et al. (1999). Facilitation of picture naming after repetitive transcranial magnetic stimulation. Neurology, 53(8), 1806-1812.

Mottaghy, F. M., Sparing, R., \& Topper, R. (2006). Enhancing picture naming with transcranial magnetic stimulation. Behavioral Neurology, 17(3-4), 177-186.

Naeser, M. A., Martin, P. I., Nicholas, M., Baker, E. H., Seekins, H., Kobayashi, M., et al. (2005). Improved picture naming in chronic aphasia after TMS to part of right Broca's area: an openprotocol study. Brain and Language, 93(1), 95-105.

Nixon, P., Lazarova, J., Hodinott-Hill, I., Gough, P., \& Passingham, R. (2004). The inferior frontal gyrus and phonological processing: an investigation using rTMS. Journal of Cognitive Neuroscience, 16(2), 289-300.

Parker, G. J., Luzzi, S., Alexander, D. C., Wheeler-Kingshott, C. A., Ciccarelli, O., \& Lambon Ralph, M. A. (2005). Lateralization of ventral and dorsal auditory-language pathways in the human brain. Neuroimage, 24(3), 656-666.

Poldrack, R. A. (2006). Can cognitive processes be inferred from neuroimaging data? Trends Cognitive Science, 10(2), 59-63.

Postma, A. (2000). Detection of errors during speech production: a review of speech monitoring models. Cognition, 77(2), 97-132.

Sakai, K. L., Noguchi, Y., Takeuchi, T., \& Watanabe, E. (2002). Selective priming of syntactic processing by event-related transcranial magnetic stimulation of Broca's area. Neuron, 35(6), 1177-1182.

Salmelin, R., Hari, R., Lounasmaa, O. V., \& Sams, M. (1994). Dynamics of brain activation during picture naming. Nature, 368(6470), 463-465.

Schiller, N. O., Horemans, I., Ganushchak, L., \& Koester, D. (2009). Event-related brain potentials during the monitoring of speech errors. Neuroimage, 44(2), 520-530.

Schnur, T. T., Schwartz, M. F., Kimberg, D. Y., Hirshorn, E., Coslett, H. B., \& Thompson-Schill, S. L. (2009). Localizing interference during naming: convergent neuroimaging and neuropsychological evidence for the function of Broca's area. Proceedings of the National Academy of Science. U S A, 106(1), 322-327.

Schuhmann, T., Schiller, N. O., Goebel, R., \& Sack, A. T. (2008). The temporal characteristics of functional activation in Broca's area during overt picture naming. Cortex.

Shapiro, K. A., Pascual-Leone, A., Mottaghy, F. M., Gangitano, M., \& Caramazza, A. (2001). Grammatical distinctions in the left frontal cortex. Journal of Cognitive Neuroscience, 13(6), 713720. 



\section{CHAPTER 5}

\section{THE FUNCTIONAL ROLE OF INFERIOR}

FRONTAL SULCUS IN DECEPTION:

\section{A NEURONAVIGATED THETA-BURST}

\section{TRANSCRANIAL MAGNETIC STIMULATION} STUDY ${ }^{1}$

${ }^{1}$ Based on: Schuhmann, T.*, Verschuere, B.*, \& Sack, A.T. (in preparation). The functional role of inferior frontal sulcus in deception: A neuronavigated theta-burst transcranial magnetic stimulation study.

* the authors contributed equally to the manuscript 


\begin{abstract}
By definition, lying involves withholding the truth. Response inhibition may therefore be the cognitive function at the heart of deception. In line with this notion, neuroimaging research has shown that the same brain region that is activated during response inhibition tasks, namely the inferior frontal region, is also activated during deception paradigms. These findings lead to the hypothesis that in the context of deception, the inferior frontal region is the neural substrate critically involved in withholding the truth. To examine the functional necessity of the inferior frontal region in deception, we experimentally manipulated the neural activity level in the inferior frontal sulcus (IFS) using a novel protocol of repetitive transcranial magnetic stimulation (rTMS), continuous theta burst stimulation (cTBS). Individual structural magnetic resonance brain images (MRI) were used to allow precise stimulation of the inferior frontal lobe in each participant. Sixteen participants answered autobiographical questions truthfully or deceptively before and after placebo (sham) or real cTBS. Deception was reliably associated with longer response times than truth telling. The cTBS induced virtual lesion in the IFS did not affect performance. Implications for the role of the IFS in response inhibition and deception are discussed.
\end{abstract}




\section{INTRODUCTION}

Many deception detection techniques rely upon the idea that deception is associated with strong emotional responding. The exact nature of this responding has often not been articulated or has been loosely and variously referred to with concepts like stress, arousal, fear, fight/flight or guilt. The straightforward hypothesis was that deception would evoke stronger emotional responding compared to truth-telling. A large number of studies have tested predictions derived from this hypothesis with regard to verbal, non-verbal, and physiological reactions (DePaulo et al., 2003). The overall conclusion of this work, however, is somewhat disappointing: The association between deception and emotional responding proves to be modest, at best. Deception is not always associated with emotional responding, whereas truth-telling may be. As a result, the validity of deception detection techniques such as voice stress analysis, Screening Passengers by Observation Technique (SPOT), or the Comparison Question polygraph Technique ("lie detector") is highly questionable. Absent a strong psychological theory on deception, technological advancement is unlikely to lead to more accurate lie detection (Meijer et al., 2009).

In recent years, deception researchers have focused upon the cognitive processes involved in deception. Formulated broadly, the cognitive perspective on deception departs from the idea that deception is cognitively more demanding than truth telling (Vrij, 2008). Deception often involves one or more of the following mental operations: the decision to lie, withholding the truth, fabrication of the lie, monitoring whether the receiver believes the lie and, if necessary, adjusting the fabricated story, and keeping the lying consistent. These operations make lying a cognitively demanding task. Indirect evidence supports the cognitive perspective on deception. For example, lying participants were judged by observers to think harder than truthful participants, and participants subjectively reported more cognitive load when lying compared to truth telling (Vrij, Fisher, Mann, \& Leal, 2006). Furthermore, compared to truth telling, lying is associated with an increase in response time (Sartori, Agosta, Zogmaister, Ferrara, \& Castiello, 2008; Verschuere, Prati, \& De Houwer, 2009). Recently, several studies used brain imaging techniques such as fMRI (Abe et al., 2008; Ganis, Kosslyn, Stose, Thompson, \& Yurgelun-Todd, 2003; Kozel et al., 2005; Langleben et al., 2002; Monteleone et al., 2006; Phan et al., 2005; Spence et al., 2001), PET (Abe et al., 2006), and fNIRS (Tian, Sharma, Kozel, \& Liu, 2009) to identify which brain regions are associated with deception. Common across these studies is the greater activation in the prefrontal cortex during lying compared to truth telling (Christ, Essen, Watson, Brubaker, \& McDermott, 2009), thereby supporting the idea that deception requires greater executive control than truth telling.

Since deception by definition involves withholding the truth, response inhibition may be the cognitive function at the heart of deception. Indeed, liars may or may not overtly express a deceitful answer, but they definitely need to refrain from telling the truth. Response inhibition can be defined as the cognitive function that allows one to intentionally inhibit a dominant, automatic or prepotent response (Miyake et al., 2000). The truth, then, is regarded as the dominant response that 
needs to be actively inhibited in order to lie (Spence, Kaylor-Hughes, Farrow, \& Wilkinson, 2008). Noteworthy from the perspective of the association between response inhibition and deception, is the observation that the same brain regions are critically involved in response inhibition and in deception. Examining the neural correlates of response inhibition, imaging studies have examined brain activity during tasks that require active suppression of a dominant response such as the Go/No-Go task and the Stop-signal task. The Go/No-Go task requires a speeded response to frequently presented Go trials (e.g., the letter Q), but inhibition of responding to the rarely presented No-Go trials (e.g., the letter 0 ). In the Stopsignal, responding to the go task (e.g., press left for circle, and right for square) has to be inhibited when an auditory signal is presented. A particular region in the prefrontal cortex, the right inferior frontal region, is consistently and most strongly activated during such tasks (Aron, Robbins, \& Poldrack, 2004; Brass, Derrfuss, Forstmann, \& von Cramon, 2005; Garavan, Ross, \& Stein, 1999; Konishi et al., 1999). In 18 patients with right frontal lobe damage, it was found that the greater the damage to the inferior frontal gyrus (IFG), the worse response inhibition performance in the Stop-signal task (Aron, Fletcher, Bullmore, Sahakian, \& Robbins, 2003). Further support for the functional necessity of the IFG in response inhibition comes from recent work using repetitive transcranial magnetic stimulation (rTMS). rTMS is a non-invasive brain stimulation technique that allows to induce a transient and reversible "virtual lesion". rTMS to the IFG, but not to mid frontal or parietal regions, impaired response inhibition capacity in healthy volunteers (Chambers et al., 2006). As the inferior frontal region is also consistently activated in deception paradigms (Christ, Essen, Watson, Brubaker, \& McDermott, 2009; Gamer, Bauermann, Stoeter, \& Vossel, 2007; Kozel et al., 2005; Phan et al., 2005; Spence et al., 2001), it may be this region that is responsible for inhibiting the truth during deception (Spence et al., 2004). However, since imaging studies are in essence correlation studies, they do not allow conclusions with regard to the functional necessity of brain regions. In order to really investigate the functional necessity of this region for deception, one would need to experimentally manipulate its activity level and investigate the impact on deception (Luber, Fisher, Appelbaum, Ploesser, \& Lisanby, 2009; Sack, 2006).

Here, we present the first study that used rTMS to unravel the functional relevance of the right inferior frontal region for deception. Following recent imaging data (Brass, Derrfuss, Forstmann, \& von Cramon, 2005), we focused upon the right inferior frontal sulcus (IFS). We collected structural images of the brain using magnetic resonance imaging (MRI). These individual anatomical brain structure images were used as a basis for a frameless stereotaxic TMS neuronavigation system, allowing us to precisely map and target the IFS with TMS in each individual. Furthermore, we used an innovative TMS protocol, continuous theta-burst rTMS (cTBS) that requires a much shorter stimulation time yet leads to more robust inhibitory after-effects than conventional rTMS protocols (Huang, Edwards, Rounis, Bhatia, \& Rothwell, 2005; Thut \& Pascual-Leone, 2010). In sum, we applied MRI-guided cTBS neuronavigation to transiently disrupt neural processing in the right IFG to gather direct evidence for the functional role of IFG in deception. 


\section{EXPERIMENT 1: BEHAVIOURAL STUDY}

Our rTMS design requires repeated (i.e., 4x) assessment of the deception task: Pre and post real and placebo (sham) stimulation. No study to date, however, has assessed the effect of such repeated testing on the validity of the deception test. We were concerned that through practice, the difference in response times between deception and truth telling would disappear. Experiment 1 sought to investigate whether valid repeated assessment of the deception test is possible and did therefore not include any rTMS stimulation, yet. Trying to circumvent practice effects, we used comparable, but different sentences in the pre and post test.

\section{EXPERIMENT 1: METHOD}

\section{Participants}

Fifteen right-handed students ( 5 male; $M$ age $=23.33$ years, $S D=2.35$ years, range 20 to 25 years) from the Psychology and Neurosciences Department of Maastricht University received course credits for participation. Prior to the experiment, informed consent was obtained from all participants. The study was approved by the Department's Ethical Commission.

\section{Apparatus}

Stimuli were presented by a PC using Inquisit 3.0 software (Inquisit, 2009). This software also recorded RTs with millisecond accuracy.

\section{Procedure}

Testing occurred individually in a sound attenuated room in two sessions, separated by a $M$ of 5.19 days ( $S D=4.37$ days; range 1 to 14 days). The procedure for the two days was identical. After signing informed consent, participants filled in an autobiographical questionnaire. This questionnaire listed 72 specific behaviours (e.g., "Bought a newspaper?", "Travelled by train?", "Had a bath?"), and participants were asked to indicate whether they had performed those actions that day by marking them Yes or No. Half of these questions came from the study by Spence and colleagues (Spence et al., 2001) the remaining half were developed for the present study. Next, participants performed the deception test (Spence et al., 2001) a first time. Trials consisted of statements from the autobiographical questionnaire presented in white in the middle of a black screen for 5s. Participants answer the statements with a right-hand Yes or No response. The Yes and No reminder labels remain on screen throughout the test. Crucially, their colour varied after every 6 trials. One colour (e.g., yellow) indicated the participant to answer truthfully, whereas the other (e.g., blue) was the signal to lie, with colours counterbalanced across participants. Meaning of the colours was assigned in the instructions, and checked in a practice phase with statements for which ground truth was known (e.g., "Are you in France?", "Are you in the Netherlands?"). The test consisted of 72 trials, with each of 36 statements appearing once with blue and once with yellow reminder labels. After a 5 minute break, participants took the deception test again, this time without practice at the beginning. One set of 36 questions was 
used in the first test, and one set in the second test, with sets counterbalanced across participants. These sets were tested beforehand to result in a deception effect of similar magnitude.

\section{EXPERIMENT 1: RESULTS}

A separate $2 \times 2 \times 2$ ANOVA with day 1 vs. day2, pre vs. post and lie vs. truth as the within-subjects factors was conducted on error percentage (\%) and on correct response times (RTs).

\section{ERROR ANALYSIS}

Responses that did not match with the autobiographical questionnaire were considered behavioural errors. Deception was associated with more errors than truth telling, $F(1,14)=14.06, p<.01$, and participants tended to make more errors in the first session of the day compared to the second session of that day, $F(1,14)=$ $3.17, p=.10$, see Table 1 . No other effects reached significance.

Table 1. Mean percentage of errors (\%; with standard deviations between parentheses) for lie and truth trials for each session in Experiment 1.

\begin{tabular}{llllll}
\hline & & Day 1 & & \multicolumn{2}{c}{ Day 2 } \\
& Truth & & Lie & Truth & Lie \\
\hline Session 1 & 5.66 & 9.29 & 2.43 & 6.24 \\
& $(7.70)$ & $(8.81)$ & $(3.01)$ & $(9.29)$ \\
Session 2 & 5.22 & 8.15 & 7.92 & 10.25 \\
& $(4.21)$ & $(6.33)$ & $(18.71)$ & $(16.46)$ \\
\hline
\end{tabular}

Table 2. Mean response latencies (in ms; with standard deviations between parentheses) for lie and truth trials for each session in Experiment 1.

\begin{tabular}{lllll}
\hline & & Day 1 & & \multicolumn{2}{c}{ Day 2 } \\
& Truth & Lie & Truth & Lie \\
\hline Session 1 & 1583 & 1713 & 1397 & 1519 \\
& $(424)$ & $(378)$ & $(407)$ & $(432)$ \\
Session 2 & 1588 & 1747 & 1449 & 1476 \\
& $(394)$ & $(402)$ & $(460)$ & $(375)$ \\
\hline
\end{tabular}

\section{$R T$ analysis}

RTs that deviated more than 2.5 SDs from the individual conditional mean (Ratcliff, 1993), and behavioural errors were excluded from the RT analyses. Participants were slower when lying than when telling the truth, $F(1,14)=17.20, p<.01$, and were slower on day1 compared to day $2, F(1,14)=15.91, p<.01$, see Table 2 . No other effects reached significance. 


\section{EXPERIMENT 1: DISCUSSION}

Experiment 1 showed that valid repeated assessment of the deception test is possible, with significant lie-truth response time differences across 4 test sessions over two days. These data confirm that the deception test could be validly applied in our rTMS design.

\section{EXPERIMENT 2: MRI GUIDED NEURONAVIGATED CTBS STUDY}

\section{METHOD}

\section{Participants}

Sixteen right-handed participants ( 2 male; $M$ age $=26.62$ years, $S D=9.44$ years, range 18 - 51 years) participated in this study and were paid $€ 15$ per hour for participation. All participants had normal or corrected-to-normal vision and had no history of neurological or psychiatric disorders. They received medical approval for participation and gave their written informed consent after being introduced to the procedure. The study was approved by the local Medical Ethical Commission.

\section{Procedure}

Participants were tested in three separate sessions. In session one, we obtained anatomical brain measurements of all participants using MRI. We performed a surface reconstruction to recover the spatial structure of the cortical sheet based on the white-grey-matter boundary. We then identified the right inferior frontal lobe by visual inspection. Specifically, for every participant we placed the TMS target point at the posterior part of IFS. This target site was saved as a digital anatomical target point for each participant (see Figure 1).

In session 2, participants were informed about the experiment and rTMS, signed informed consent, filled in the autobiographical questionnaire, and performed the deception test a first time. Next, we used single-pulse TMS to the left motor cortex to determine the active motor threshold (AMT) in every participant. We dosed the rTMS intensity relative to this individual AMT by applying TMS with $80 \%$ of AMT. We then used frameless stereotaxy for MRI-guided TMS neuronavigation to the previously defined target region, and applied either a CTBS protocol that has shown to inhibit the stimulated areas for up to 1 hours following the TBS itself (Huang, Edwards, Rounis, Bhatia, \& Rothwell, 2005; Thut \& Pascual-Leone, 2010), or sham TBS using a placebo TMS coil. The placebo coil mimics the clicking sound of stimulation without actively interfering with brain activity. To maximise rTMS effects, the second deception test followed immediately after the rTMS/Sham and did not include a practice phase. The procedure in session 3 was identical to session 2 , except that stimulation type differed and that motor threshold was not determined again. Real rTMS stimulation was on session 1 for 9 participants and on session 2 for 7 participants. 


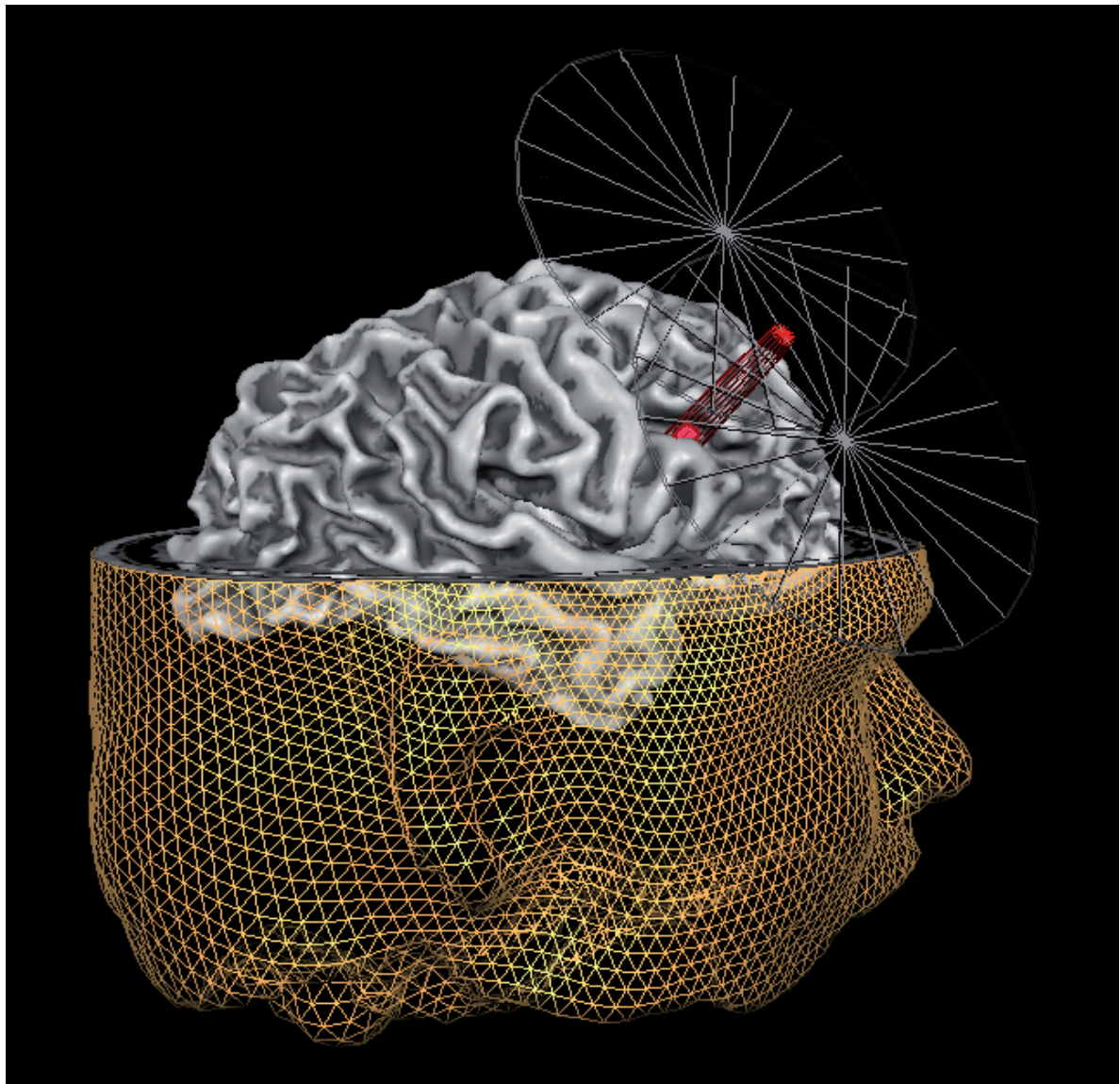

Figure 1. TMS neuronavigation based on an anatomically defined target point.

The inferior frontal sulcus (IFS) target point (red dot under the beam of the coil) for TMS, shown on the reconstruction of the right hemisphere of one exemplary participant. The target point is placed on the posterior part of the right IFS, in particular the area just anterior to the section of the precentral sulcus and the inferior frontal sulcus. In addition to the reconstruction of the right hemisphere of this participant, also the reconstruction of the head is displayed together with a simplified visualisation of the coil. The tip of the red beam from the TMS figure-eight coil indicates the site of the maximal stimulation.

This study design and methodological approach enabled us to first define the target brain area based on the individual anatomical data and to subsequently neuronavigate the TMS coil to the anatomically defined stimulation sites in each participant. The MRI-guided TMS neuronavigation was monitored online throughout the whole stimulation time, allowing for a precise determination of the actual stimulation site during stimulation. Except for the rTMS/Sham stimulation and its preparation (e.g., AMT, neuronavigation), the study design of sessions 2 and 3 was identical to that of Experiment 1. 


\section{MRI MEASUREMENTS}

A high-resolution anatomical image was obtained from each participant in a 3-T magnetic resonance scanner (Siemens Allegra MR Tomograph; Siemens AG, Erlangen, Germany) at the Faculty of Psychology and Neuroscience, Maastricht University, The Netherlands. The data set was acquired with the help of a T1-weighted structural scan with an isotropic resolution of $1 \mathrm{~mm}$ using a modified driven equilibrium Fourier transform (MDEFT) sequence with optimised contrast for GM and WM and imaging parameters.

\section{CORTICAL-SURFACE RECONSTRUCTION}

Data were analysed using the BrainVoyager QX 2.0 software package (BrainInnovation, Maastricht, The Netherlands). The high-resolution anatomical recordings were used for surface reconstruction of the left hemisphere of each participant (Kriegeskorte \& Goebel, 2001). The surface reconstruction was performed in order to recover the exact spatial structure of the cortical sheet and to improve the visualisation of the anatomical gyrification. The white-grey-matter boundary was segmented with a region growing method preceded by inhomogeneity correction of signal intensity across space. The borders of the two resulting segmented subvolumes were tessellated to produce a surface reconstruction of the left hemisphere.

\section{CO-REGISTRATION OF STEREOTAXIC AND MRI DATA}

For a precise positioning of the stimulation coil, we made use of the BrainVoyager TMS Neuronavigator (BrainInnovation, Maastricht, The Netherlands). This neuronavigator system consists of several miniature ultrasound transmitters which are attached to a participant's head, as well as the TMS coil. The ultrasound markers continuously transmit ultrasonic pulses to a receiving sensor device. The measurement of the relative spatial position of these transmitters in 3-D space is based on travel time of the transmitted ultrasonic pulses to three microphones of the receiving sensor device. Local spatial coordinate systems are created by linking the relative raw spatial position of the ultrasound senders to a set of fixed additional landmarks on the participant's head. The specification of these fixed landmarks is achieved via a digitising pen that also hosts two transmitting ultrasound markers in order to measure its relative position in 3-D space. The three anatomical landmarks we used to define the local coordinate system were the nasion and the two incisurae intertragicae. The neuronavigation system then provides topographic information of the head ultrasound transmitters relative to a participant-based coordinate frame. Similarly, the TMS coil also hosts a set of ultrasound transmitters whose relative spatial positions are linked to fixed landmarks specified on the coil in order to calculate another local coordinate system. After having defined the local spatial coordinate system for the participant's head and the TMS coil in real 3-D space, these coordinate systems have to be co-registered with the coordinate system of the MR space. For TMS-MRI co-registration, the same landmarks digitised on the participant's head are specified on the head reconstruction of the anatomical data from the MR sequence. After the landmarks specified on the real head have been 
co-registered with those on the reconstructed head, events occurring around the head of the participant in real space are registered online and visualised in realtime at correct positions relative to the participant's anatomical reconstruction of the brain. The TMS coil can now be neuronavigated to a specific anatomical area of each participant. TMS neuronavigation was based on data in AC-PC space (rotating the cerebrum into the anterior commissure - posterior commissure plane) in order to avoid any additional transformations that could distort the correspondence between MRI and stereotaxic points.

\section{TMS APPARATUS AND STIMULATION PARAMETERS}

Biphasic TMS pulses were applied using the MagProX100 stimulator (Medtronic Functional Diagnostics A/S, Sklovunde, Denmark) and a figure-of-eight coil (MC$B 70$, inner radius $10 \mathrm{~mm}$, outer radius $50 \mathrm{~mm}$ ) for real stimulation. The maximum output of this coil and stimulator combination is approximately 1.9 Tesla and 150 A/ $\mu \mathrm{S}$. A specific figure-of-eight placebo coil (MC-P-B70) was also employed in order to reproduce the same acoustic stimulation as the active coil while not inducing the magnetic field (sham stimulation). The coil was manually held tangentially to the skull with the coil handle oriented perpendicular to the posterior part of the inferior frontal sulcus using the online visualisation function of the BrainVoyager TMS Neuronavigator. Continuous theta burst (CTBS) TMS was applied at $80 \%$ active motor threshold. Detailed descriptions of this rTMS paradigm have been given elsewhere (Huang et al., 2005). In brief, TBS consists of trains of 3 TMS pulses (triplet) that are separated by $20 \mathrm{~ms}$ ( $50 \mathrm{~Hz}$ frequency), with each train being repeated every $200 \mathrm{~ms}(5 \mathrm{~Hz})$. Depending on the inter-triplet-interval, TBS can either have longer lasting facilitatory or inhibitatory after effects, which has been refereed to as intermittent (im) versus continuous (c) TBS. In a cTBS protocol, the $50 \mathrm{~Hz}$ triplets are applied in one continuous train with a $5 \mathrm{~Hz}$ rhythm with a total duration of, in our case, 40 seconds, i.e. 600 pulses.

\section{TMS LOCALISATION}

IFS corresponds to area 44 in Brodmann's cytoarchitectonic map (Brodmann, 1909). We targeted the posterior part of the right IFS, in particular the area just anterior to the section of the precentral sulcus and the inferior frontal sulcus. This stimulation site was localised using the BrainVoyager TMS Neuronavigator (see Figure 1).

\section{TMS PROCEDURE}

Individual active motor thresholds (AMTs) were determined as the intensity at which the stimulation of the left motor cortex with single-pulse TMS resulted reliably in a visible movement of the FDI. The AMT of the participants ranged from $21 \%$ to $45 \%$ of maximum stimulator output $(M=30.50 \%$ [ $47 \mathrm{~A} / \mu \mathrm{S}], S D=5.97)$. The mean stimulation intensity was set at $80 \%$ of the AMT and therefore resulted in $24.67 \%(38.5 \mathrm{~A} / \mu \mathrm{S})$ of maximum stimulator output (range $17 \%$ to $36 \%$, SD = 4.97). Throughout the stimulation time, participants were wearing earplugs to pro- 
tect their ears from the clicking sound and to minimise the interference of the sounds during the task

\section{EXPERIMENT 2: RESULTS}

A separate $2 \times 2 \times 2$ ANOVA with rTMS vs. Sham, pre vs. post and lie vs. truth as the within-subjects factors, was conducted on error percentage (\%) and on correct response times (RTs). Data preparation was identical to that of Experiment 1.

\section{Error analysis}

The main effect of deception, $F(1,15)=8.11, p<.05$ subsumed under the deception by pre/post interaction, $F(1,15)=5.44, p<.05$. Breaking down this interaction it appeared that participants made more errors when lying compared to truth telling in the pre test, $t(15)=3.29, p<.01$, but not the post test, $t<1$. No other effects reached significance.

\section{$R T$ analysis}

There was a main effect of deception, with participants being slower when lying than when telling the truth, $F(1,15)=23.36, p<.001$. No other effects reached significance. However, there was a trend towards a three way interaction of rTMS vs. Sham, pre vs. post, and lie vs. truth, $F(1,15)=2.23, p=.16$, with only truth responses tending to slow down after real stimulation, $t(15)=1.40, p=.18$. This effect, however, failed to reach statistical significance (see Figure 2).
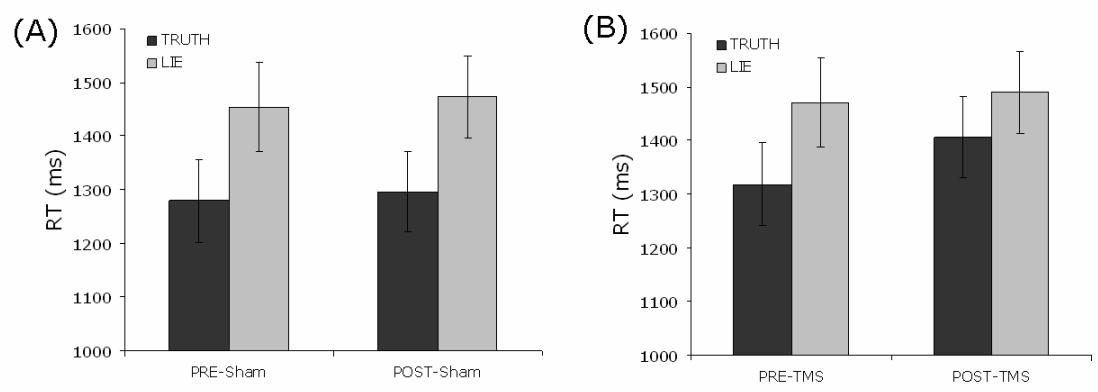

\section{Figure 2. Mean RTs for SHAM and REAL stimulation.}

Mean RTs in ms (plus standard errors) before and after SHAM (A) and real TMS (B), shown separately for truth and lie trials.

\section{EXPERIMENT 2: DISCUSSION}

The data of Experiment 2 again confirmed the validity of the deception test, with significant lie-truth response time differences. The rTMS, however, had no significant influence on the response times. 


\section{GENERAL DISCUSSION}

Although badly needed by private (e.g. insurance) companies, and law enforcement agencies worldwide, no full proof "lie detector" exists at present. As long as we are still missing a solid cognitive theory on deception, technological advancement is unlikely to lead to more accurate lie detection (Meijer et al., 2009). Deception researchers now focus upon the cognitive aspects involved in deception. According to this theory, and fundamental for the current study, deception involves two cognitive characteristics: first, deception seems cognitively more demanding than truth telling, and second, the truth seems to be an automatic response that actively needs to be inhibited during lying (Sartori, Agosta, Zogmaister, Ferrara, \& Castiello, 2008; Verschuere, Prati, \& De Houwer, 2009; Vrij, Fisher, Mann, \& Leal, 2006). Since deception by cognitive definition involves withholding the truth, response inhibition may be the cognitive function at the heart of deception. The truth is regarded as the dominant response that needs to be actively inhibited in order to lie (Spence, Kaylor-Hughes, Farrow, \& Wilkinson, 2008), and that this response inhibition of the truth is regulated mainly in right inferior frontal cortex (Christ, Essen, Watson, Brubaker, \& McDermott, 2009; Gamer, Bauermann, Stoeter, \& Vossel, 2007; Kozel et al., 2005; Phan et al., 2005; Spence et al., 2001; Spence et al., 2004). However, since imaging studies are in essence correlation studies, they do not allow conclusions with regard to the functional necessity of brain regions. In order to really investigate the functional necessity of this region for deception, one would need to experimentally manipulate its activity level and investigate the impact on deception (Luber, Fisher, Appelbaum, Ploesser, \& Lisanby, 2009; Sack, 2006). Here, we present the first study that used rTMS to unravel the functional relevance of the right inferior frontal cortex for deception. Following recent imaging data (Brass, Derrfuss, Forstmann, \& von Cramon, 2005), we focused upon the right inferior frontal sulcus (IFS). We collected structural images of the brain using magnetic resonance imaging (MRI). These individual anatomical brain structure images were used as a basis for a frameless stereotaxic TMS neuronavigation system, allowing us to precisely map and target the IFS in each individual participant. Furthermore, we used an innovative TMS protocol, continuous theta-burst rTMS (cTBS), that requires a much shorter stimulation time yet leads to more robust inhibitory after-effects than conventional rTMS protocols (Huang, Edwards, Rounis, Bhatia, \& Rothwell, 2005; Thut \& Pascual-Leone, 2010).

The psychophysics results of our two studies both showed that lying was reliably associated with greater response times and more errors compared to truth telling. These data support the idea that deception is cognitively more demanding than telling the truth and that lying thus comes with a cognitive "cost", as measured by increased reaction times and error rates. With respect to these behavioural patterns of lying versus truth telling, the present study is the first to demonstrate that this cost can be reliably measured within participants across several assessments. Based on these stable psychophysics findings, we were interested to investigate whether a cTBS-induced disruption of the right IFS would significantly modify these behavioural measures of deception. Specifically, we expected that a TMS- 
induced disruption of right IFS would hinder the inhibition of the truth during lying, thereby leading to increased reaction times and/or error rates in the lying trials. Our TMS findings, however, failed to support this notion and did not reveal any significant evidence that the IFS is critically involved in withholding the truth. In fact, the experimental manipulation of the brain activity in this region did not alter behaviour in the deception paradigm, nor in the lying nor in the truth telling trials. The only descriptive trend noticeable in our TMS data is a slight increase in reaction times after cTBS over IFS for telling the truth. Interestingly, this is also evident in the conducted ANOVA where we found a statistical trend for a three-way interaction of rTMS vs. Sham, pre vs. post, and lie vs. truth with only truth responses tending to slow down after real stimulation. But, this effect failed to reach statistical significance.

Hence, although our study was based on a clear a priori hypothesis directly derived from the imaging literature, and conducted using state-of-the art TMS methodology - including 1 ) the employment of individual structural brain imaging data to select and target the IFS in each individual participant, 2) a paradigm that reliably elicits stronger inferior frontal activation for lying compared to truth telling (Spence et al., 2001; Spence et al., 2004; Spence, Kaylor-Hughes, Brook, Lankappa, \& Wilkinson, 2008), and 3) a stimulation protocol (cTBS) that has been shown to produce immediate, profound and lasting effects on cognitive functioning (Huang, Edwards, Rounis, Bhatia, \& Rothwell, 2005; Thut \& Pascual-Leone, 2010)-, our TMS study failed to reveal any significant main or interaction effects.

This raises the question of how, and if at all, to interpret these null findings with regard to the underlying cognitive theory on deception, and the interpretability of TMS null findings in general. In a recent opinion paper, de Graaf and Sack (in review) provide a possible framework for the conceptual considerations of TMS null results along a gradient of meaningfulness, including several recommendations on experimentation and interpretation. The authors mainly discriminate between three possible (counter) arguments for the meaningful interpretation of null results in TMS studies, namely (1) the localisation argument, (2) the neural efficacy argument, and (3) the power argument. With regard to the localisation argument, they suggest that TMS null findings are most problematic when it remains uncertain whether the coil was positioned properly and the intended targeted brain region were therefore actually stimulated with TMS. We believe that this argument does not hold in the current study. Here, we used an individual MRI-guided TMS Neuronavigation approach, and in accordance with our a priori hypothesis aimed to target right IFS in every single participant. The online and real-time frameless TMS neuronavigation system used in our study ensured that TMS was exactly positioned over this particular brain region during cTBS in each single participant. Hence, we did know and quantified exactly where we stimulated and would thus be confident in interpreting our null findings with respect to the functional relevance of this particular stimulation site. Please note, however, that this does by no means rule out the possibility that we might have revealed other results when stimulating at other prefrontal target sites. However, as for this study it is save to conclude that when stimulating the exact target point within right IFS, no TMS effects are revealed. In 
this sense, we could interpret our findings in such a way and claim that our data provide empirical support for the notion that right IFS is not functionally relevant during deception. However, the neural efficiency and power arguments are less easily countered in our study and might thus still make such a conclusion questionable. It may very well be that the lack of significant effects seen in our study is simply brought about by the fact that our correctly identified target region within right IFS was not properly stimulated by our TMS protocol. This could mean that the intensity was too low, that the ongoing neural process was too robust to be significantly affected by TMS, or that the parameters were ill-chosen (according to de Graaf \& Sack, in review, the neural efficiency argument). Finally, there is the power argument, stating that the present study may simply have lacked the power to find significant effects. At this point, we do see a statistical trend for a 3-way interaction showing that cTBS does affect the RT for telling the truth. We cannot rule out that by testing more participants this trend will eventually become statistically significant. However, even if this was the case, the direction of the induced effect would be opposite to our a priori expectation. We speculated that IFS represents the suppression of the truth during lying. Applying CTBS to the IFS should make lying slower because the automatic tendency for the dominant truth response cannot be adequately inhibited anymore. However, we did find the exact opposite, suppressing IFS with cTBS increased RT for truth telling while not affecting lying. Hence, even if we were to find that with increasing $\mathrm{N}$ this effect would reach statistical significance (the power argument), we could only post hoc speculate about a possible interpretation of the direction of these TBS-induced behavioural changes in our deception paradigm. One of these speculation leads us directly to the above mentioned neural efficiency argument. Although continuous theta-burst rTMS (cTBS) has been shown to induce strong, robust and reliable inhibitory after-effects as measured by decreased cortical excitability when trying to induce MEP's following CTBS in the motor cortex (Huang, Edwards, Rounis, Bhatia, \& Rothwell, 2005; Thut \& Pascual-Leone, 2010), the current study could not provide any direct evidence that we indeed induced these exact neurophysiological after effects also at our stimulation site and in our participant cohort. Unlike for the motor system, no direct and easily measurable assessment for the local cortical excitability level of right IFS is available, unless cTBS is directly combined with EEG or fMRI during stimulation. Hence, our TBS-induced inhibition of right IFS is a mere assumption based on previous work using this TMS paradigm over motor regions. It has been shown that there is a considerable inter-individual variance in the cortical after effects of rTMS (Maeda, Keenan, Tormos, Topka, \& Pascual-Leone, 2000) with some participants showing an increase in cortical excitability while others showing a respective decrease in cortical excitability, even when being stimulated with the same rTMS protocol (Maeda, Keenan, Tormos, Topka, \& Pascual-Leone, 2000). Moreover, it has been shown that the same rTMS protocol can induce opposite neural after effects (excitatory versus inhibitory) when applied over different cortical target sites. Hence, as long as a direct neurophysiologic measurement of our cTBS intervention is missing, we have no direct evidence whether we really induced a suppression or excitation of right IFS. 
Yet, aside the problem of whether we successfully inhibited right IFS with our TBS protocol we also only rely on an assumption when it comes to interpreting the increased activity in right IFS as revealed by functional imaging studies. When interpreting functional imaging results and TMS findings, we in essence aim to incorporate three different sources of information: the transient local changes of neural activity during the execution of a certain task, the physiological modulation of this activity by TMS, and the behavioural consequences of the magnetic stimulation. When an area of the brain is activated during a cognitive process, and a TMSinduced modulation of the neural activation of this area leads to a significant change of task performance, we might assume that this area is functionally relevant for this specific cognitive function. Yet, a TMS-induced impairment of task performance can be brought about by four different effect chains: the magnetic stimulation can lead to the inhibition of an area that supports task execution, or to the excitation of an area that inhibits this function, or to the inhibition of an area that inhibits processes that compete with the respective function, or the excitation of an area that promotes competing functions (Sack \& Linden, 2003). To what extent the increase in BOLD signal during deception really does reflect inhibition or excitation remains unknown in itself. The BOLD signal in fact rather represents intracortical processing (Logothetis, Pauls, Augath, Trinath, \& Oeltermann, 2001; Logothetis \& Wandell, 2004), and response inhibition might thus appear in functional imaging as an area with increased, decreased, or no BOLD signal changes during task execution (Ritter \& Villringer, 2002). In other words, the fact that - if statistically reliable with increased $\mathrm{N}$ - our CTBS protocol resulted in an increase in RT for telling the truth could theoretically be brought about by a variety of possible neural mechanism. First, it may be that right IFS does not inhibit but support truth telling, and that thus inhibiting IFS with cTBS increased specifically RT for truth trials. Second, IFS may inhibit the lie and thus disinhibiting the inhibition of the lie results in slowing down truth telling. In any case, in order to empirically address all of these possible interpretations in a step-wise falsification procedure, it is needed that different, ideally opposite, TMS protocols, such as CTBS versus imTBS, are applied at different nodes of the entire deception network, while simultaneously assessing the influence of opposite TMS-induced neural effects on deception behaviour and neural network activity as measured, e.g., by concurrent EEG and/or fMRI.

To conclude, although we can be sure which exact stimulation site we stimulated in our current study, we still cannot make any concrete conclusions regarding the null findings when stimulating this region with cTBS during a deception paradigm. In our case the absence of evidence is still no evidence of absence because we, at this point, can not convincingly address the above mentioned neural efficiency and power argument for an interpretability of TMS null findings. Yet, our paradigm, methodological set-up and preliminary findings seem promising enough, to proceed with this research line. We suggest to address the neural efficiency and power argument by 1 ) increasing number of participants, and 2) expanding the TMS procedure to different opposite TMS protocols and a parametric variation of the most relevant TMS parameters such as intensity and frequency. If successful, this research hold the potential to reveal most exciting insights into the functional net- 
work accounts of deception, and frontal inhibition in social interaction, with wide ranging implications for fundamental brain research and society.

\section{ACKNOWLEDGEMENTS}

Bruno Verschuere is a postdoctoral fellow of the Scientific Research Foundation (FWO). Teresa Schuhmann and Alexander T. Sack were supported by a grant from the Netherlands Organization for Scientific Research (NWO; grant number 452-06$003 \& 400-04-215)$. We thank our medical supervisor Cees van Leeuwen, and our independent physician Martin van Boxtel. 


\section{REFERENCES}

Abe, N., Okuda, J., Suzuki, M., Sasaki, H., Matsuda, T., Mori, E., et al. (2008). Neural Correlates of True Memory, False Memory, and Deception. Cerebral Cortex, 18(12), 2811-2819.

Abe, N., Suzuki, M., Tsukiura, T., Mori, E., Yamaguchi, K., Itoh, M., et al. (2006). Dissociable roles of prefrontal and anterior cingulate cortices in deception. Cerebral Cortex, 16(2), 192-199.

Aron, A. R., Fletcher, P. C., Bullmore, E. T., Sahakian, B. J., \& Robbins, T. W. (2003). Stop-signal inhibition disrupted by damage to right inferior frontal gyrus in humans. Nature Neuroscience, 6(2), 115-116.

Aron, A. R., Robbins, T. W., \& Poldrack, R. A. (2004). Inhibition and the right inferior frontal cortex. Trends in Cognitive Sciences, 8(4), 170-177.

Brass, M., Derrfuss, J., Forstmann, B., \& von Cramon, D. Y. (2005). The role of the inferior frontal junction area in cognitive control. Trends in Cognitive Sciences, 9(7), 314-316.

Brodmann, K. (1909). Vergleichende Lokalisationslehre der Großhirnrinde in ihren Prinzipien dargestellt auf Grund des Zellenbaues: Leipzig: Johann Ambrosius Barth.

Chambers, C. D., Bellgrove, M. A., Stokes, M. G., Henderson, T. R., Garavan, H., Robertson, I. H., et al. (2006). Executive "brake failure" following deactivation of human frontal lobe. Journal of Cognitive Neuroscience, 18(3), 444-455.

Christ, S. E., Essen, D. C., Watson, J. M., Brubaker, L. E., \& McDermott, K. B. (2009). The Contributions of Prefrontal Cortex and Executive Control to Deception: Evidence from Activation Likelihood Estimate Meta-analyses. Cerebral Cortex, 19(7), 1557-1566.

DePaulo, B. M., Lindsay, J. J., Malone, B. E., Muhlenbruck, L., Charlton, K., \& Cooper, H. (2003). Cues to deception. Psychological Bulletin, 129(1), 74-118.

Gamer, M., Bauermann, T., Stoeter, P., \& Vossel, G. (2007). Covariations Among fMRI, Skin conductance and behavioral data during processing of concealed information. Human Brain Mapping, 28, 1287-1301.

Ganis, G., Kosslyn, S. M., Stose, S., Thompson, W. L., \& Yurgelun-Todd, D. A. (2003). Neural correlates of different types of deception: An fMRI investigation. Cerebral Cortex, 13(8), 830-836.

Garavan, H., Ross, T. J., \& Stein, E. A. (1999). Right hemispheric dominance of inhibitory control: An event-related functional MRI study. Proceedings of the National Academy of Sciences of the United States of America, 96(14), 8301-8306.

Huang, Y. Z., Edwards, M. J., Rounis, E., Bhatia, K. P., \& Rothwell, J. C. (2005). Theta burst stimulation of the human motor cortex. Neuron, 45(2), 201-206.

Inquisit. (2009). (Version 3.0). Seattle, WA: Millisecond software.

Konishi, S., Nakajima, K., Uchida, I., Kikyo, H., Kameyama, M., \& Miyashita, Y. (1999). Common inhibitory mechanism in human inferior prefrontal cortex revealed by event-related functional MRI. Brain, 122, 981-991.

Kozel, F. A., Johnson, K. A., Mu, Q. W., Grenesko, E. L., Laken, S. J., \& George, M. S. (2005). Detecting deception using functional magnetic resonance imaging. Biological Psychiatry, 58(8), 605-613.

Kriegeskorte, N., \& Goebel, R. (2001). An efficient algorithm for topologically correct segmentation of the cortical sheet in anatomical mr volumes. Neuroimage, 14(2), 329-346.

Langleben, D. D., Schroeder, L., Maldjian, J. A., Gur, R. C., McDonald, S., Ragland, J. D., et al. (2002). Brain activity during simulated deception: An event-related functional magnetic resonance study. Neuroimage, 15(3), 727-732.

Logothetis, N. K., Pauls, J., Augath, M., Trinath, T., \& Oeltermann, A. (2001). Neurophysiological investigation of the basis of the fMRI signal. Nature, 412(6843), 150-157.

Logothetis, N. K., \& Wandell, B. A. (2004). Interpreting the BOLD signal. Annual Review of Physiology, $66,735-769$.

Luber, B., Fisher, C., Appelbaum, P. S., Ploesser, M., \& Lisanby, S. H. (2009). Non-Invasive Brain Stimulation in the Detection of Deception: Scientific Challenges and Ethical Consequences. Behavioral Sciences \& the Law, 27(2), 191-208.

Maeda, F., Keenan, J. P., Tormos, J. M., Topka, H., \& Pascual-Leone, A. (2000). Interindividual variability of the modulatory effects of repetitive transcranial magnetic stimulation on cortical excitability. Experimental Brain Research, 133(4), 425-430. 
Meijer, E. H., Verschuere, B., Vrij, A., Merckelbach, H., Smulders, F., Leal, S., et al. (2009). A call for evidence-based security tools. Open Access Journal of Forensic Psychology, 1, 1-4.

Miyake, A., Friedman, N. P., Emerson, M. J., Witzki, A. H., Howerter, A., \& Wager, T. D. (2000). The unity and diversity of executive functions and their contributions to complex "frontal lobe" tasks: A latent variable analysis. Cognitive Psychology, 41(1), 49-100.

Monteleone, G. T., Phan, K. L., Fitzgerald, D. A., Irick, J. S., Hawkley, L. C., \& Cacioppo, J. T. (2006). Can lying be diagnosed using fMRI? Psychophysiology, 43, S68-S68.

Phan, K. L., Magalhaes, A., Ziemlewicz, T. J., Fitzgerald, D. A., Green, C., \& Smith, W. (2005). Neural correlates of telling lies: A functional magnetic resonance imaging study at 4 tesla. Academic Radiology, 12, 164-172.

Ratcliff, R. (1993). Methods for Dealing with Reaction-Time Outliers. Psychological Bulletin, 114(3), 510-532.

Ritter, P., \& Villringer, A. (2002). Inhibition and functional magnetic resonance imaging. International Congress Series, 1235, 213-222.

Sack, A. T. (2006). Transcranial magnetic stimulation, causal structure-function mapping and networks of functional relevance. Current Opinion in Neurobiology, 16(5), 593-599.

Sack, A. T., \& Linden, D. E. (2003). Combining transcranial magnetic stimulation and functional imaging in cognitive brain research: possibilities and limitations. Brain Research. Cognitive Brain Research, 43(1), 41-56.

Sartori, G., Agosta, S., Zogmaister, C., Ferrara, S. D., \& Castiello, U. (2008). How to accurately assess autobiographical events. Psychological Science, 19, 772-780.

Spence, S. A., Farrow, T. F. D., Herford, A. E., Wilkinson, I. D., Zheng, Y., \& Woodruff, P. W. R. (2001). Behavioural and functional anatomical correlates of deception in humans. Neuroreport, 12(13), 2849-2853.

Spence, S. A., Hunter, M. D., Farrow, T. F. D., Green, R. D., Leung, D. H., Hughes, C. J., et al. (2004). A cognitive neurobiological account of deception: evidence from functional neuroimaging. Philosophical Transactions of the Royal Society of London Series B-Biological Sciences, 359(1451), 1755-1762.

Spence, S. A., Kaylor-Hughes, C., Farrow, T. F. D., \& Wilkinson, I. D. (2008). Speaking of secrets and lies: The contribution of ventrolateral prefrontal cortex to vocal deception. Neuroimage, 40(3), 1411-1418.

Spence, S. A., Kaylor-Hughes, C. J., Brook, M. L., Lankappa, S. T., \& Wilkinson, I. D. (2008). 'Munchausen's syndrome by proxy' or a 'miscarriage of justice'? An initial application of functional neuroimaging to the question of guilt versus innocence. European Psychiatry, 23(4), 309-314.

Thut, G., \& Pascual-Leone, A. (2010). A Review of Combined TMS-EEG Studies to Characterize Lasting Effects of Repetitive TMS and Assess Their Usefulness in Cognitive and Clinical Neuroscience. Brain Topography, 22(4), 219-232.

Tian, F. H., Sharma, V., Kozel, F. A., \& Liu, H. L. (2009). Functional near-infrared spectroscopy to investigate hemodynamic responses to deception in the prefrontal cortex. Brain Research, 1303, 120-130.

Verschuere, B., Prati, V., \& De Houwer, J. (2009). Cheating the lie detector: Faking in the autobiographical IAT. Psychological Science, 20, 410-413.

Vrij, A. (2008). Detection of lies and deceit: Pitfalls and Opportunities. Second Edition. Chichester: John Wiley \& Sons Ltd.

Vrij, A., Fisher, R., Mann, S., \& Leal, S. (2006). Detecting deception by manipulating cognitive load. Trends in Cognitive Sciences, 10(4), 141-142. 


\section{SUMMARY AND CONCLUSION}



The work presented in this thesis had a main goal of widening our knowledge of using transcranial magnetic stimulation (TMS) in cognitive studies. The particular points of interest were the testability of silent areas with TMS, the use of neuronavigation and the optimisation of coil positioning in cognitive studies. Furthermore, we were interested in causal chronometry and the involvement of networks in higher cognition.

Chapter 2 assessed the influence of different TMS coil positioning approaches on TMS-induced behavioural effects in cognitive studies. Four commonly used TMS coil positioning approaches were systematically compared by using them to induce behavioural change in a single cognitive study. Specifically, the behavioural impact of right parietal TMS during a number comparison task was assessed, while basing TMS localisation either on 1) individual functional magnetic resonance imaging (fMRI) -guided TMS Neuronavigation, 2) individual magnetic resonance imaging (MRI) -guided TMS Neuronavigation, 3) group functional Talairach Coordinates, or 4) an anatomical landmark approach using the 10-20 EEG electrode positioning system. The findings revealed a systematic difference between the four approaches, with the individual fMRI-guided TMS Neuronavigation yielding the strongest and the 10-20 EEG system approach yielding the smallest behavioural effect size. Accordingly, power analyses revealed that while in the fMRI-guided neuronavigation approach five participants were sufficient to reveal a significant behavioural effect, the number of necessary participants increased to $n=9$ when employing MRI-guided neuronavigation, to $\mathrm{n}=13$ in case of TMS based on group Talairach coordinates, and to $n=47$ when applying TMS over electrode position P4 according to the 10-20 EEG system. These graded effect size differences between TMS coil positioning strategies and the also documented inter-individual variances in the actual target stimulation site within and between approaches are of direct relevance for any study aiming to target a specific "silent" brain area with TMS, for example within the parietal or frontal cortex. Accordingly, the findings of this study were applied to Chapter 3-5, and in all of the following chapters an optimised coil positioning was used.

In Chapter 3, the testability of higher cognitive silent areas in the brain with TMS was probed. Here, the functional role as well as the precise temporal involvement of Broca's area during language production, as taking place during picture naming, was investigated. Broca's area is an anatomically complex region including the pars opercularis (F3Op), the pars triangularis (F3Tr) and the pars orbitalis, which show anatomical variability between participants. Individual anatomical mapping and frameless stereotaxy was used to precisely map and target Broca's area in each individual participant. Event-related triple-pulse TMS (tpTMS) was applied online while participants were performing a picture naming task at various time points after picture presentation. This enabled us to not only receive information about whether Broca's area is functionally relevant during picture naming, but to also exactly identify at which precise point in time the neural activity in Broca's area contributes to the processes of picture naming. To control for effects caused by other than the direct magnetic stimulation of the brain, sham stimulation was included. Applying online triple-pulse TMS over Broca's area resulted in an increase 
in RTs in a picture naming task only when applied $300 \mathrm{~ms}$ after picture presentation. Thus by using online, time-resolved TMS we were able to not only demonstrate that Broca is functionally relevant for speech production but were also able to chart at what exact time point this activity is critical.

After having obtained information about the functional involvement of Broca's area as well as the timing of the functional activity, the question arose as to what role other language-related brain areas play in the process of language production. Particularly the relative causal chronometry within and between several nodes of the highly distributed brain language network was of interest. We therefore probed the behavioural relevance of Broca's area, Wernicke's area, and the midsection of the left middle temporal gyrus (MTG) for successful language production by directly interfering with their local neural activity changes online, i.e., during overt picture naming, using MRI-guided chronometric TMS. This enabled us to test whether neural activity in these regions is functionally relevant for picture naming and to chart the exact time point at which this neural activity is critical. We applied MRI-guided TMS while participants were performing a picture naming task (online) and at various time points between picture onset and overt speech production (at 150ms, $225 \mathrm{~ms}, 300 \mathrm{~ms}, 400 \mathrm{~ms}$ and $525 \mathrm{~ms}$ after picture presentation). We demonstrated that the midsection of the left MTG becomes functionally relevant first at $225 \mathrm{~ms}$ post-picture-onset, followed by Broca's area at 300 ms, and Wernicke's area at 400 ms. Interestingly, during this late time window, MTG shows a second peak of functional relevance. Each area thus contributed at different stages during the speech production process, suggesting distinct underlying functional roles within this complex, multi-componential skill. We proposed that the first peak of functional relevance in MTG represents the early process of word retrieval. This information is then processed in Broca's area for subsequent phonological encoding, which in turn back-projects the phonologically encoded word to MTG, resulting in its second peak of functional relevance. At the same time, the retrieved information is forwardprojected to Wernicke's area for internal self-monitoring. We thus proposed a neurobiological model of speech production according to which successful speaking relies on intact, spatiotemporally specific feed-forward and recurrent feedback loops within a left-hemispheric fronto-temporal brain network.

Finally, using a novel patterned repetitive TMS (rTMS) protocol we tried to interfere with yet another highly cognitive process, namely deception. The role of the right inferior frontal sulcus (IFS) in deception was of special interest to us since neuroimaging research has shown that the same brain region that is activated during response inhibition tasks, namely the inferior frontal region, is also activated during deception paradigms. These findings led to the hypothesis that in the context of deception, the inferior frontal region is the neural substrate critically involved in withholding the truth. The innovative TMS protocol, continuous thetaburst rTMS (CTBS) that requires a much shorter stimulation time yet leads to more robust inhibitory after-effects than conventional rTMS protocols, was used to interfere with IFS functioning. Participants had to answer autobiographical questions truthfully or deceptively before and after placebo (sham) or real cTBS over right IFS. Deception was reliably associated with longer response times than truth telling. 
However, the cTBS induced virtual lesion in the IFS was not found to affect performance reliably.

In conclusion, the empirical studies presented in this thesis provide concrete experimental evidence for the notion that the non-invasive brain interference technique of TMS is capable of interfering with neural activity in various silent cortical brain regions. This TMS-induced neural disruption can lead to specific and reliably measurable impairments in higher cognitive functions. In this sense, TMS, when applied as an online event-related protocol, represents a suitable means to chart the time course of functional relevance in studies of higher cognition. However, the empirical work of this thesis also revealed that the inter-individual variability in brain anatomy and, more importantly, the inter-individual variability in the exact structure-function correspondence, is a much more pressing issue than, for example, in TMS studies targeting primary sensory brain regions within the primary motor (M1) or visual cortex (V1). In other words, unlike TMS over M1 or V1, the higher inter-individual variability of structure-function correspondence seen in parietal or frontal cortices requires an optimised TMS coil positioning system, which, as shown here, should ideally be based on individual brain imaging data. Yet, more sensory or sensorimotor research questions may still be addressed with TMS in the absence of such imaging-guided strategies.

This thesis also showed that despite the question of functional relevance per se, online event-related TMS is also capable of charting the exact time point at which neural activity in a given brain region is critical for successful task performance. By applying such a TMS paradigm over several nodes of the same widely distributed brain network underlying speech production, we charted the relative time points of functional necessity in each of these network nodes, documenting a certain temporal order of functional relevance between distinct brain regions. Our interpretation of these relative timing differences in functional contribution assumed a temporal sequence of information flow in which one area processes a certain aspect of the task and subsequently sends this information to another brain region for further processing. Although our interpretation includes possible feedforward and feedback loops and recurrent information flow, it is important to note that such interpretation of effective brain connectivity based on chronometric TMS data is by definition implicit. The chronometric TMS results do not provide direct evidence of feedforward or feedback flow of information, but they do show timing differences in functional relevance between brain regions within one network, thereby providing strong but indirect evidence regarding neural information flow. To empirically complement such evidence for a particular effective connectivity model, it would be most interesting and useful to evaluate some family of effective connectivity models by combining TMS chronometry with the evidence using, for example, structural equation modelling or dynamic causal modelling, and/or Granger causality mapping.

Finally, we now have several non-invasive techniques at hand to not only reliably and accurately measure human brain activity during task execution (watch the brain at work), but to even non-invasively manipulate this human brain activity during task execution and to assess the consequences of such experimentally- 
induced brain disturbances on behaviour and cognition. Yet we are still left emptyhanded when it comes to the concrete meaning of all the measured and revealed information. Despite all the technological power inherent to brain imaging and brain interference techniques, they do not provide us with the concrete cognitive label of what we see or what we disturb. Several brain regions may be activated during the execution of a complex cognitive task and TMS over these regions may specifically impair the performance in this task at particular points in time. Yet it still remains a matter of interpretation and speculation by the individual researcher as to which exact cognitive sub processes are represented by these brain regions. In these times of modern experimental brain research, the expertise and knowledge of the researcher conducting such brain imaging or brain interference studies, determines the quality and usefulness of the revealed results, and it is her/his knowledge of the field and scientific creativity that makes sense out of the data, gives cognitive labels to the neural networks, and thus provides meaning to our findings. In particular for TMS research, where a certain brain region is targeted at a specific time point during a particular behavioural paradigm, a well-motivated hypothesis and cognitive model is required and vital for success. In fact, the design of TMS studies forces us to formulate concrete expectations about which brain region is involved in which cognitive function at which temporal stage a priori, including a concrete cognitive model of the paradigm, as derived from previous research or models of cognitive psychology. In this sense, also in these times of modern brain imaging and brain interference techniques, the fundamental principles of epistemology are still, and maybe even more, relevant and valid and should be strictly applied to Cognitive Neuroscience. 


\section{SAMENVATTING EN CONCLUSIE}



Het voornaamste doel van het hier voorgelegde werk is de vergroting van onze kennis op het terrein van het gebruik van transcraniële magnetische stimulatie (TMS) binnen de cognitieve neurowetenschappen. Met name is dit werk specifieker gericht op het onderzoek van 'silent areas' ('stille hersengebieden' - hersengebieden waar stimulatie niet onmiddellijk leidt tot zichtbare effecten), het gebruik van neuronavigatie, en daarbij vooral op het optimaliseren van spoelpositionering in cognitieve studies. Ook waren we geïnteresseerd in causale chronometrie en het belang van hersennetwerken in hogere cognitie.

Hoofdstuk 2 onderzocht de invloed van verschillende TMS-spoelpositioneringsmethoden op TMS-gedragseffecten in cognitieve studies. We vergeleken systematisch vier veelgebruikte methoden, door ze toe te passen in eenzelfde cognitieve studie. De gevolgen van TMS over de rechter pariëtaalkwab op de uitvoering van een getallenvergelijkingstaak werden vergeleken in vier verschillende methodologische scenario's. TMS-localisatie van het relevante pariëtale gebied was gestoeld op: 1) neuronavigatie geleid door individuele functionele magnetische resonantie imaging (fMRI) beelden, 2) neuronavigatie geleid door individuele anatomische magnetische resonantie imaging (MRI) beelden, 3) functionele groepscoördinaten in het Talairach kader, 4) anatomische eikpunten volgens het internationale 10-20 EEGpositioneringssysteem.

Op deze manier konden we een systematisch verschil aantonen tussen deze vier positioneringsmethoden. De individuele fMRI-geleide TMS-neuronavigatie had hierbij het grootste, en het 10-20 EEG-systeem het kleinste effect op de gedragsmaat. Poweranalyses lieten zien dat met de fMRI-geleide TMS-neuronavigatie slechts 5 proefpersonen nodig zijn voor een significant TMS-effect, met de MRIgeleide navigatie 9 proefpersonen, terwijl met de groepscoördinaten in Talairach een totaal van 13 mensen, en met de 10-20 EEG-localisatie een totaal van 47 mensen mee moeten doen aan het onderzoek om dezelfde TMS-effecten te vinden.

Deze graduele verschillen in TMS effect grootte tussen de verschillende positioneringsmethoden, evenals de gevonden inter-individuele variabiliteit in de locaties van de werkelijk gestimuleerde hersengebieden per methode, zijn van direct belang voor elke TMS-studie naar 'silent brain areas', zoals de frontale of pariëtale hersengebieden. Wij hebben de inzichten uit dit onderzoek toegepast op de hierop volgende studies in hoofdstuk 3-5, en dus een optimale spoelpositioneringsmethode toegepast.

In Hoofdstuk 3 bekeken we de mogelijkheid tot onderzoek van hogere cognitieve, stille, hersengebieden met TMS. In dit onderzoek evalueerden we zowel de functionele rol als de relevantie in de tijd van Broca's gebied tijdens taalproductie, middels een taak waarin plaatjes benoemd moesten worden. Broca's gebied is een anatomisch complex gebied, dat de pars opercularis (F3Op), de pars triangularis (F3Tr), en de pars orbitalis bevat, structuren die anatomisch aanzienlijk kunnen variëren tussen proefpersonen. Individuele anatomische reconstructies van het brein en 'frameless stereotaxy' werden gebruikt om in iedere proefpersoon heel precies het gebied van Broca te verstoren met TMS.

'Event-related triple-pulse' TMS (tpTMS) werd online toegepast, dat wil zeggen terwijl proefpersonen plaatjes benoemden, op verschillende tijdstippen na het aan- 
bieden van een visueel plaatje. Deze opzet maakte het niet alleen mogelijk om informatie te verzamelen over het belang van Broca's gebied voor spraakproductie, maar ook om precies te bepalen op welk moment Broca's gebied bijdraagt aan het benoemen van een plaatje.

Om te controleren of de gevonden effecten inderdaad een specifiek gevolg waren van de magnetische stimulatie, werd dezelfde procedure toegepast met 'sham' stimulatie, waarbij Broca's gebied niet werkelijk gestimuleerd werd maar alle andere TMS-effecten zoals bijvoorbeeld het geluid nagebootst werden. Online tpTMS over Broca's gebied resulteerde in langere reactietijden in de spraakproductietaak wanneer de stimulatie 300 milliseconden na presentatie van het plaatje plaatsvond. Door het toepassen van online TMS waarbij de timing gevarieerd werd, waren we dus niet alleen in staat de functionele relevantie van Broca's gebied vast te stellen, maar ook de exacte tijd waarop de activiteit in dit gebied cruciaal is voor spraakproductie.

$\mathrm{Na}$ het verwerven van deze data over de functionele betrokkenheid van Broca's gebied bij spraakproductie en de bijbehorende timing van deze functionele activiteit, deed zich de vraag voor: Welke rol spelen andere bekende taalgebieden bij de taalproductie? Hierbij was de relatieve causale chronometrie van verscheidene gebieden binnen het wijdverspreide taalnetwerk in het brein van belang. In een nieuw experiment onderzochten we daarom de relevantie van Broca's gebied, Wernicke's gebied en het middengedeelte van de linker middelste temporale gyrus (MTG) voor succesvolle taalproductie, door middels MRI-geleide chronometrische TMS 'online' (tijdens het plaatjes benoemen) de neurale activiteit in die gebieden te verstoren.

Dit stelde ons in staat om te evalueren of de neurale activiteit in deze gebieden werkelijk relevant is voor het benoemen van afbeeldingen en wanneer de activiteit relevant is. Hiertoe stimuleerden we tijdens de uitvoering van de taak met MRI-geleide TMS de verschillende gebieden op verscheidene momenten tussen het aanbieden van het plaatje en het benoemen ervan door de proefpersoon (d.w.z. op $150 \mathrm{~ms}, 225 \mathrm{~ms}, 300 \mathrm{~ms}, 400 \mathrm{~ms}$ en $525 \mathrm{~ms}$ na aanbieding van het plaatje). We konden aantonen dat de midsectie van MTG relevant is vanaf $225 \mathrm{~ms}$, maar dat Broca's gebied pas relevant wordt op 300 ms en Wernicke's area zelfs pas vanaf $400 \mathrm{~ms}$.

Interessant genoeg liet MTG een tweede piek van functionele relevantie zien in dit late tijdsbestek. Ieder taalgebied draagt dus bij aan het spraakproductieproces in verschillende stadia, hetgeen suggereert dat er verschillende functionele rollen toebedeeld zijn binnen dit complexe multi-componentiële proces in het brein. Wij suggereerden dat de eerste piek van MTG correspondeert met een vroeg proces van 'word retrieval'; het ophalen van een uit te spreken woord. Deze informatie wordt vervolgens verwerkt in Broca's gebied voor fonologische encodering; het samenstellen van de klanken van dat woord. Dit samengestelde woord wordt daarna weer teruggestuurd naar MTG, wat diens tweede piek in relevantie verklaart. Tegelijkertijd wordt al deze informatie gestuurd naar Wernicke's gebied voor interne controle en registratie. Samengevat stelden we dus een neurobiologisch model van spraakproductie voor, waarin succesvol spreken afhankelijk is van intacte, 
spatiotemporeel specifieke feedforward en feedback loops binnen een frontotemporaal netwerk in de linker hersenhelft.

Tenslotte gebruikten we een nieuw repetitief TMS (rTMS) protocol in een poging een ander hoog-cognitief proces te verstoren, namelijk deceptie. Wij waren vooral geïnteresseerd in de rol van de rechter inferieure frontale sulcus (IFS) bij deceptie, aangezien neuroimaging onderzoek heeft aangetoond dat hetzelfde hersengebied dat actief is tijdens respons-inhibitietaken, namelijk de inferieure frontale regio, ook actief is tijdens deceptie. Dit gegeven leidde tot de hypothese dat, in de context van deceptie, de inferieure frontale regio het neurale substraat is van het achterhouden van de waarheid.

Het innovatieve TMS-protocol dat we gebruikten voor het verstoren van IFS, genaamd 'continuous theta-burst' TMS (cTBS), maakt een veel kortere stimulatietijd mogelijk dan klassieke rTMS protocollen, maar heeft desalniettemin langdurigere en meer robuuste inhibitoire na-effecten. Proefpersonen werden gevraagd om autobiografische vragen naar waarheid of misleidend te beantwoorden, na placebo (sham) of werkelijke cTBS over het inferieur frontale gebied IFS. Deceptie leidde tot langere reactietijden dan het vertellen van de waarheid. Echter, de virtuele laesie van IFS, geïnduceerd door cTBS, had geen betrouwbaar effect op het gedrag.

Samenvattend kan gesteld worden dat de hier gepresenteerde empirische studies laten zien dat niet-invasieve verstoring van de hersenactiviteit middels TMS gebruikt kan worden om 'silent areas' (waaronder prefrontale en inferieur temporale gebieden) in het brein te onderzoeken en om vast te stellen wat hun rol is bij het tot stand brengen van hogere cognitieve processen en gedrag. Tevens is TMS in staat, indien toegepast als online event-related protocol, om voor een gegeven hersengebied het tijdsverloop van functionele relevantie voor hogere cognities in kaart te brengen.

Het empirische werk in dit proefschrift laat echter ook zien dat interindividuele variabiliteit in hersenanatomie en, nog belangrijker, interindividuele variabiliteit in de relatie tussen structuur en functie een groter probleem vormen voor cognitieve studies dan voor, bijvoorbeeld, TMS-studies naar primaire sensorische gebieden als de motor cortex (M1) en visuele hersenschors (V1). Met andere woorden, in tegenstelling tot TMS over M1 of V1, zorgt grote variabiliteit in de relatie tussen structuur en functie tussen proefpersonen (geobserveerd voor frontale en pariëtale cortex) dat een geoptimaliseerde spoelpositioneringsmethodiek vereist is voor nauwkeurig experimenteel werk, dat dan bij voorkeur gebaseerd is op individuele neuroimaging data. TMS-onderzoek naar meer primaire gebieden kan wellicht evengoed uitgevoerd worden zonder deze geavanceerde methodiek.

Dit proefschrift laat ook zien dat, naast de vraag over de functionele relevantie, online event-related TMS in staat is om het tijdsverloop van relevantie van neurale activiteit in een gegeven hersengebied voor een bepaalde taak in kaart te brengen. Door een dergelijk TMS paradigma toe te passen op verschillende gebieden die deel uitmaakten van eenzelfde, wijdverspreide netwerk in het brein, konden wij de relatieve tijdspunten van functionele relevantie van al deze netwerkgebieden blootleggen en daardoor een bepaalde temporele volgorde van relevantie van die gebieden schetsen. 
Onze interpretatie van deze relatieve tijdsverschillen in functionele bijdrage ging uit van een temporele opeenvolging van informatieverwerking waarin een bepaald hersengebied een gegeven aspect van de gehele taak verwerkt en vervolgens de resultaten doorstuurt naar andere hersengebieden voor verdere verwerking. Hoewel onze interpretatie daarmee mogelijk feedforward en feedback loops en wederkerige informatiestromen behelst, is het belangrijk om op te merken dat zulke interpretaties aangaande effectieve connectiviteit in het brein, voor zover gebaseerd op chronometrische TMS-data, bij voorbaat impliciet zijn.

De chronometrische TMS-resultaten geven geen direct bewijs voor feedforward of feedback informatiestromen, maar laten wel zien dat er verschillen zijn tussen de tijdstippen waarop verschillende hersengebieden belangrijk zijn voor een taak. Dat vormt interessant, zij het indirect, bewijs voor neurale informatiestromen. Om een resulterend neurobiologisch model van effectieve hersenconnectiviteit empirisch aan te vullen zou het interessant en nuttig zijn om effectieve connectiviteitsmodellen op basis van TMS-chronometrie te combineren en te vergelijken met andere effectieve connectiviteitsmethoden zoals, bijvoorbeeld, 'structural equation modelling' of 'Granger causality mapping'.

Concluderend hebben we tegenwoordig verscheidene niet-invasieve technieken ter beschikking die niet alleen betrouwbaar en nauwkeurig de menselijke hersenactiviteit kunnen meten tijdens taakuitvoering ('kijken naar het werkende brein'), maar ook technieken die in staat zijn om niet-invasief deze activiteit te beïnvloeden tijdens de taakuitvoering, om vervolgens de gevolgen van dergelijke experimentele manipulaties op gedrag en cognitie te evalueren. Maar we staan nog altijd met lege handen als het gaat om de concrete betekenis van alle gemeten en blootgelegde informatie. Ondanks alle technologische vooruitgang gaan neuroimaging- en neuromanipulatietechnieken helaas niet gepaard met concrete cognitieve labels bij wat we zien of wat we verstoren.

Verscheidene hersengebieden kunnen actief zijn tijdens de uitvoering van een complexe taak. TMS over deze gebieden kan de uitvoering van deze specifieke taak beïnvloeden op heel specifieke tijdstippen, maar dan nog weten we niet zeker welke exacte cognitieve subprocessen we verstoord hebben in de gestimuleerde hersengebieden. Het antwoord op die vraag blijft vaak een zaak van speculatie en interpretatie en de opvattingen van de individuele onderzoeker. In de huidige tijden van modern empirisch hersenonderzoek bepaalt de expertise en kennis van de onderzoeker die het experiment uitvoert vaak de kwaliteit en bruikbaarheid van onderzoeksgegevens. Kennis van het wetenschappelijk speelveld en wetenschappelijke creativiteit geven dan vaak de doorslag als het gaat om interpretatie van de data en het plakken van cognitieve labels op neurale netwerken.

Vooral in TMS-onderzoek, waarin een gegeven hersengebied wordt gestimuleerd op een specifiek tijdstip in een specifiek gedragsparadigma, is een goed onderbouwde a priori hypothese en een duidelijk gespecificeerd cognitief model van groot belang. Sterker nog, het ontwerpen van TMS-studies dwingt ons om concrete verwachtingen te formuleren over de vraag welke hersengebieden betrokken zijn bij welke cognitieve componenten in welke van de opeenvolgende stadia. Met andere woorden, op basis van voorgaand cognitief psychologisch onderzoek moet een 
concreet cognitief model geformuleerd worden dat kan dienen als context voor het gebruikte TMS-paradigma. Op deze manier wordt duidelijk dat, zelfs in deze tijden van brain imaging en interferentie technieken, fundamentele epistemologische principes nog meer dan ooit, relevant en valide zijn voor onderzoek binnen de Cognitieve Neurowetenschap. 



Een proefschrift en de daarbij behorende onderzoeken doe je niet alleen. Daarom wil ik hier een aantal mensen bedanken.

First of all I would like to thank my supervisors Alexander Sack and Niels Schiller. Alex, thank you for being my supervisor over the last four years. From day one you gave me a lot of responsibility and let me be a part in building up the TMS lab. Your laissez faire style of guiding the TMS group lets us all work in a relaxed atmosphere, where each of us has the chance to develop and strengthen their own talents. Your problem solving attitude helped me out of little research dips many times. Thank you for teaching me how to be a researcher who does not lose the big picture.

Niels, I started as a student assistant with you in my third year, my first contacts with real science. I will never forget when I, after mentioning once that I might be interested in a PhD, but only when it includes TMS, got an email from you in San Diego saying that all is sorted and that I will be able to do my PhD with you and Alex when I get back from the States. Thank you for giving me the chance to do a PhD with you. It is a pity that you moved to Leiden during my PhD which meant that the group language meetings became quite rare. But your supervision continued via email and I am very pleased with the result of our work together.

It was great working in such a motivating and stimulating environment as $\mathrm{CN}$ provides. Thanks to all of you, who are part of this, in particular I would like to thank those seniors that I had contact with, namely Elia, Peter and Rainer. Furthermore, my thank goes out to the TMS group. I still remember the first meetings with only Alex, Nina and me in Alex' office. These times are long over and the group is ever growing. Joel, Nina, Christianne, Tom, Felix, Vincent, and Tommaso, I am very happy to be working in a group with all of you. I think that we are the only group that actually manages to have meeting of two hours when nothing has to be discussed. De helft van de TMS groep heft mij geholpen bij de vertaling van mijn samenvatting, Tom, Joel, Nina en Christianne, bedankt hiervoor. Tom, wat goed dat we je oren inmiddels gewassen hebben. Ik had niet gedacht dat het zo plezierig zou zijn om met jou samen te werken. Joel, bedankt voor al jouw hulp met Brain Voyager gerelateerde vragen, paper vragen, en je luisterend oor voor al mijn verhaaltjes.

Bernie, durch Dich habe ich Forschung in meinem zweiten Studienjahr kennengelernt. Danke für all Deine Unterstützung vor allem während meines Amerikaaufenthaltes, aber auch davor und danach.

Verder wil ik ook nog mijn collega-vrienden, Gonny, Anniek, Petra, Dries, Jeannette en Noel danken. Ik heb het altijd zo gezellig met jullie en vind het erg fijn dat ik met jullie onder de lunch en ook op alle andere gelegenheden over andere dingen dan werk kan praten (maar dus ook wel over werk als het even moest).

Vera, es war schön immer zu wissen, dass ich hier süddeutsche Unterstützung habe, die eben noch weiß, wie alles begann. Betsy, gut zu wissen, dass ich immer nur kurz rufen muss oder eben ein paar Schritte gehen um wissenschaftliche aber noch viel mehr persönliche Dinge mit jemanden besprechen zu können. Thanks to Martin, Giancarlo and Francesco for not only making life at work a lot nicer, but especially for all the great nights with great food and lots of laughter. 
Lieve Rob, mijn kamergenoot, hoewel ik met jou op een kamer zit is het mij gelukt om mijn these af te krijgen! Ik ben heel blij om jou als kamergenoot te hebben. Bedankt voor alle gezellige en ook serieuze gesprekken "met de deur dicht". Fijn dat ik je nog een tijdje hier niet moet missen.

Danken wil ik ook het secretariaat van $\mathrm{CN}$, met name Annemie. Bedankt voor al je ondersteuning in financiële en organisatorische dingen. De jongens van Instrumentatie hebben mij ook veel geholpen tijdens mijn promotietijd. Hier wil ik vooral Johan, René en Eric danken. Fijn dat jullie bij elk telefoontje uit het TMS lab meteen naar beneden kwamen om mij te helpen. Ook dank aan de mannen van de vijfde, de IT jongens voor alle hulp bij computer gerelateerde problemen. Sven, bedankt voor de hulp bij het scannen en bij onze pogingen om 3 onderzoeksmethoden simultaan te gebruiken. Mijn dank gaat ook uit aan al mijn proefpersonen (voor mijn taal studies moesten ze wel wat kwaaltjes ondergaan), onze medische supervisor Cees van Leeuwen en onze onafhankelijke arts Martin van Boxtel danken.

Mario, vielen Dank für all die Hilfe beim Einplanen der Versuchspersonen einplanen, bei den Messungen und Analysen. Thanks to Vincent van de Ven, Bernadette Jansma, Felix Mottaghy and Joan Camprodon for being part of the reading committee.

Bruno, met jou samen te werken was enorm plezierig. Ongelofelijk hoe goed georganiseerd en toch heel relaxed iemand kan zijn. Ik hoop dat we nog veel projecten samen gaan doen.

Thanks to my Maastricht friends Angelica and Henrik, Katja and Ivan, Camilo and Aude for being there, taking care of Matilda, feeding us and enjoying life with us.

I would also like to thank my paranimfen and friends Anne and Alessia. Anne, thank you for being my paranimf. I am very happy to have you here in Maastricht. Thank you for babysitting Matilda, helping us move for the thousand's time, setting up our house etc. Alli, since the day that you were the only one laughing about my jokes, we have practically been inseparable. Thank you for all the trips we took, all the laughter we shared and just for always being such a good friend to me. I miss you dearly here in Maastricht and life is just not the same without you here.

Auch meiner Familie möchte ich danken. Mama und Papa, danke, dass Ihr immer für mich da seid und mich in allen meinen Wegen bedingungslos unterstützt. Maren und Anne, ich bin froh, dass ich zwei so tolle große Schwestern habe, zu denen ich immer mit all meinen Fragen und Problemen gehen kann. Maren und Mark, danke auch für Eure Hilfe beim Korrekturlesen. Ihr alle, zusammen mit Timm, Emma, Mila und Lotte seid ein guter Ausgleich zu der wissenschaftlichen Welt und helft mir sehr, diese Welt auch mal zu relativieren.

Matilda, Du kamst und gabst allen Dingen eine ganz neue Dimension. Alex, Du bist mein Alpha und mein Omega. 


\section{CURRICULUM VITAE}


Teresa Schuhmann was born on September $15^{\text {th }} 1979$ in Scherzingen, Switzerland. She attended the Alexander von Humboldt Gymnasium in Konstanz, Germany, where she obtained her Abitur in 1998. In 2000 she commenced to study psychology at Maastricht University, where she became a student research assistant on ERP studies together with Prof. Dr. Niels Schiller in 2002. In this period she specialised in Neuropsychology. In 2004, she did a research internship at the University of California, San Diego supervised by Prof. Dr. Marta Kutas and Prof. Dr. Bernadette Jansma. In 2005, she received her Master's degree in Psychology from Maastricht University. In 2005 she started working for Dr. Alexander Sack and Prof. Dr. Niels Schiller as a research assistant. In 2006, she continued her work as a PhD student at the Faculty of Psychology and Neuroscience in Maastricht, section Cognitive Neuroscience under the supervision of Dr. Alexander Sack and Prof. Dr. Niels Schiller. Currently, she is employed as a postdoctoral fellow at the Department of Cognitive Neuroscience, Faculty of Psychology, Maastricht University. 


\section{PUBLICATIONS}

Schuhmann, T., Schiller, N. O., Goebel, R., \& Sack, A. T. (2009). The temporal characteristics of functional activation in Broca's area during overt picture naming. Cortex, 45, 1111-1116.

Sack, A. T., Cohen Kadosh, R., Schuhmann, T., Moerel, M., Walsh, V., \& Goebel, R. (2009). Optimizing functional accuracy of TMS in cognitive studies: a comparison of methods. Journal of Cognitive Neuroscience, 21, 207-221.

Sack, A. T., van der Mark, S., Schuhmann, T., Schwarzbach, J., \& Goebel, R. (2009). Symbolic action priming relies on intact neural transmission along the retino-geniculo-striate pathway. Neuroimage, 44, 284-293.

Cohen Kadosh, R., Cohen Kadosh, K., Schuhmann, T., Kaas, A., Goebel, R., Henik, A., et al. (2007). Virtual Dyscalculia Induced by Parietal-Lobe TMS Impairs Automatic Magnitude Processing. Current Biology, 17, 689-693.

Schiller, N. O., Schuhmann, T., Neyndorff, A. C., \& Jansma, B. M. (2006). The influence of semantic category membership on syntactic decisions: A study using event-related brain potentials. Brain Research, 1082, 153-164.

\section{SUBMITTED AND IN PREPARATION}

Schuhmann, T., Schiller, N. O., Goebel, R., \& Sack, A. T. (submitted). Speaking of which: Dissecting the neurocognitive network of language production.

Schuhmann, T., Verschuere, B., \& Sack, A.T. (in preparation). The functional role of inferior frontal sulcus in deception: A neuronavigated theta-burst transcranial magnetic stimulation study.

\section{CONTACT INFORMATION}

Teresa Schuhmann

Mosalunet 98

6221JM Maastricht

The Netherlands

t.schuhmann@maastrichtuniversity.nl 